\title{
Quiver origami: discrete gauging and folding
}

\section{Antoine Bourget, Amihay Hanany and Dominik Miketa}

Theoretical Physics, Department of Physics, Imperial College London, South Kensington Campus, London, SW7 2AZ, U.K.

E-mail: a.bourget@imperial,ac.uk, a.hanany@imperial.ac.uk, d.miketa16@imperial.ac.uk

ABSTRACT: We study two types of discrete operations on Coulomb branches of $3 d \mathcal{N}=4$ quiver gauge theories using both abelianisation and the monopole formula. We generalise previous work on discrete quotients of Coulomb branches and introduce novel wreathed quiver theories. We further study quiver folding which produces Coulomb branches of non-simply laced quivers. Our methods explicitly describe Coulomb branches in terms of generators and relations including mass deformations.

Keywords: Field Theories in Lower Dimensions, Global Symmetries, Supersymmetric Gauge Theory, Solitons Monopoles and Instantons

ARXIV EPRINT: 2005.05273 


\section{Contents}

1 Introduction 2

2 Preliminaries $\quad 7$

2.1 Folding of Dynkin diagrams

2.2 The monopole formula 11

$\begin{array}{lll}2.3 & \text { Abelianisation } & 13\end{array}$

2.4 Construction of Coulomb branch multiplets 14

3 Discrete gauging $\quad 16$

$\begin{array}{ll}3.1 \text { Wreath product } & 17\end{array}$

$\begin{array}{ll}3.2 & \text { Action on the Coulomb branch } \\ & 17\end{array}$

3.3 Wreathed quivers 21

3.4 Monopole formula for wreathed quivers 23

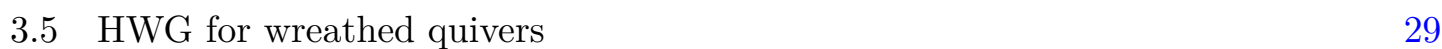

3.6 Higgs branch of wreathed quivers 33

3.7 Mirror symmetry and discrete gauging 37

4 Quiver folding 38

4.1 Action on the Coulomb branch 39

4.2 Monopole formula: examples 41

4.2.1 $\min A_{3} \rightarrow \min C_{2} \quad 41$

4.2.2 $\min D_{4} \rightarrow \min G_{2}$

4.3 Non-simply laced quivers 44

5 Examples $\quad \mathbf{4 5}$

$5.1 \min A_{3} \rightarrow$ (n.)min $C_{2}$

$\begin{array}{lll}5.1 .1 & \text { Initial quiver } & 46\end{array}$

$\begin{array}{lll}5.1 .2 & \text { Discrete gauging } & 47\end{array}$

$\begin{array}{lll}\text { 5.1.3 Folding } & 47\end{array}$

$5.2 \min D_{4} \rightarrow G_{2} \quad 48$

$\begin{array}{lll}5.2 .1 & \text { Initial quiver } & 51\end{array}$

$\begin{array}{lll}5.2 .2 & \text { Folding } & 51\end{array}$

$\begin{array}{lll}5.2 .3 & S_{3} \text { discrete gauging } & 51\end{array}$

5.2.4 Mixed folding and $S_{2}$ gauging $\quad 52$

$\begin{array}{lll}5.2 .5 & \mathbb{Z}_{3} \text { discrete gauging } & 53\end{array}$

$\begin{array}{lll}5.3 \quad D_{5} \rightarrow B_{4} & 54\end{array}$

$\begin{array}{ll}\text { A } \mathrm{U}(2) \text { with } 4 \text { flavors and abelianised rings } & 58\end{array}$

B Folded Lie algebras are the same as discretely gauged Lie algebras $\quad 61$

C Computation of Hilbert series with $S_{4}$ wreathing 62 


\section{Introduction}

The purpose of this work is to clarify the relation between several concepts relating to $3 d$ $\mathcal{N}=4$ Coulomb branches. It has been known since [1] that the Coulomb branch monopole formula [2] can be extended to quivers in the form of non-simply laced framed Dynkin diagrams. However, while all the ingredients of a simply laced Dynkin diagram - gauge and flavor nodes, hypermultiplet links - are readily interpretable, it was unknown at the time what to make of the novel multiple link. Recently [3] argued that their Coulomb branches result from a discrete folding operation on Coulomb branches of simply laced quivers. We independently derive and illustrate the same claim through the method of abelianisation [4]. We also develop a second, related but distinct discrete operation which was previously studied in [5, 6]. Both aspects expand on our previous work in [7] and likewise present an effective approach for explicit computation of generators, relations and complex mass deformations of Coulomb branches with which we solve several novel cases.

The main concepts, presented in figure 1, can be summarised as follows.

Quivers with an automorphism possess a discrete symmetry relating gauge groups. By analogy with continuous gauge groups, it, or any of its subgroups, can be gauged, ${ }^{1}$ and we demonstrate that this results in a theory whose Coulomb branch is a discrete quotient of the original, where the action by which we quotient is directly induced by the quiver automorphism (or subgroup thereof). This operation, which we call discrete gauging, produces wreathed quivers. Previous work $[5,6]$ generated similar results on the Coulomb branch by replacing $n \mathrm{U}(1)$ nodes by a $\mathrm{U}(n)$ node with adjoint matter.

In contrast, quiver folding relates Coulomb branches of pairs of simply laced and nonsimply laced quiver gauge theories. To be clear, we show the action on the Coulomb branch and conjecture that one can view it as one effect of an action on the theory. ${ }^{2}$ However, we have been unsuccessful in our attempts to write down the path integral or compute the Higgs branch of folded theories. Coulomb branches of balanced $A_{2 n-1}$-type quivers, ie. framed linear quivers satisfying the balance condition ${ }^{3}$ and exhibiting $\mathfrak{s l}(2 n, \mathbb{C})$ symmetry on the Coulomb branch, can be "folded" into Coulomb branches of balanced $C_{n}$-type quivers with $\mathfrak{u s p}(2 n, \mathbb{C})$ symmetry. Balanced $D_{n}$-type quiver Coulomb branches, ie. Coulomb branches of balanced framed quivers shaped like $D_{n}$ Dynkin diagrams, can be "folded" into Coulomb branches of balanced $B_{n-1}$-type quivers. $G_{2}$-type quiver Coulomb branches can be similarly obtained from $D_{4}$-type quivers while $F_{4}$-type quivers are folded $E_{6}$-type quivers. The folded spaces are fixed points under the group action induced by the quiver automorphism and we show that they are symplectic leaves of spaces obtained

\footnotetext{
${ }^{1}$ Discretely gauging string backgrounds is of course an old idea which has generated a lot of discussion, for example in [8-12], and our paper may be viewed as a new entry.

${ }^{2}$ In the rest of this text we will elide the distinction between folding a quiver theory and folding its Coulomb branch, but wish to be clear that we present solid evidence only for the latter and at best circumstantial evidence for the former.

${ }^{3} \mathrm{~A}$ node is balanced when the contributions of gauge and matter to the RG flow of the gauge coupling exactly cancel out assuming the quiver is understood as a $4 d$ theory. Assuming simply laced unitary quivers without loops, this amounts to the condition that twice the node's rank equals the sum of all surrounding (gauge or matter) nodes' ranks.
} 


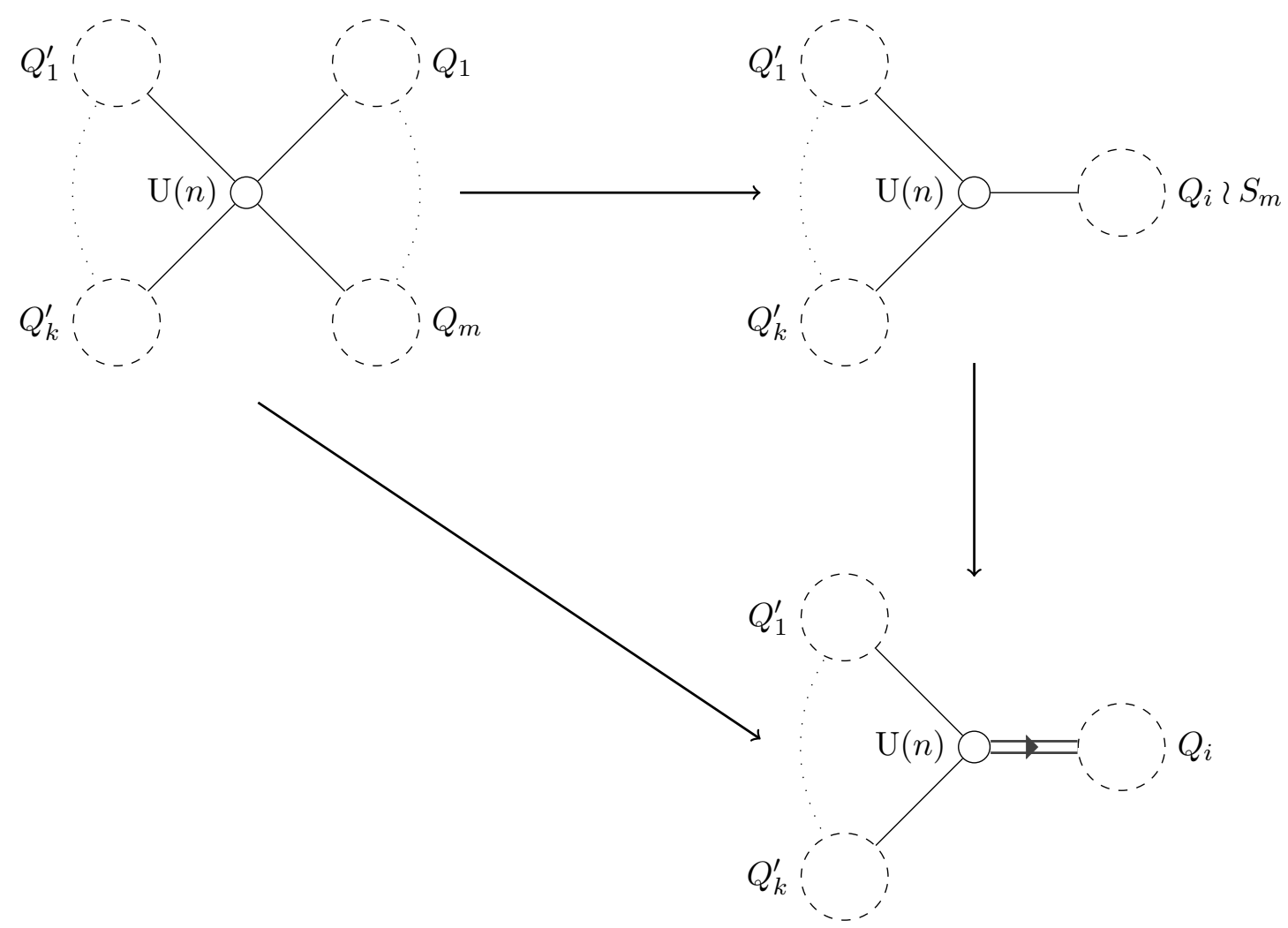

Figure 1. (Top left) $k$ generic subquivers $Q_{1}^{\prime}$ through $Q_{k}^{\prime}$ and $m$ identical subquivers $Q_{1}$ through $Q_{m}$ are connected to a common central $\mathrm{U}(n)$ node. (Top right) Wreathed quiver. (Bottom right) Non-simply laced quiver. The multiple link has valence $m$, here depicted for $m=2$.

by discretely gauging their respective original Coulomb branches. In some cases distinct subgroups of the quiver automorphism can give identical sets of fixed points (eg. $S_{3}$ and $\mathbb{Z}_{3}$ of the $D_{4}$ affine quiver) and their folded spaces coincide; as a result, there are "fewer" folded than wreathed quivers.

Actions of both discrete gauging and folding on the Coulomb branch are readily interpreted through a geometric lens, see figure 2. We claim that, since discrete gauging is implemented by restricting the chiral ring to invariants of a symmetry group action $\Gamma$, the resulting space is an orbifold of the initial Coulomb branch under $\Gamma$ - and since the Poisson structure respects this group action, the orbifold inherits a natural symplectic structure. If the original space is a nilpotent orbit of some algebra then the orbifold is sometimes, but not always, a nilpotent orbit of the relevant folded algebra, but it is in any case symmetric under the folded algebra's action.

Folding, on the other hand, reduces the Coulomb branch to the fixed subspace under the same group action $\Gamma$. We show that it has a Poisson structure and, since the fixed subspace is (a singular) part of the corresponding orbifold, the Hasse diagram [13] of the folded space is a subdiagram of the orbifold's Hasse diagram. In all known cases a nilpotent orbit folds to another nilpotent orbit (of the folded algebra). This situation is reminiscent of a general phenomenon identified in [14], in which orbits in the small affine Grassmannian 

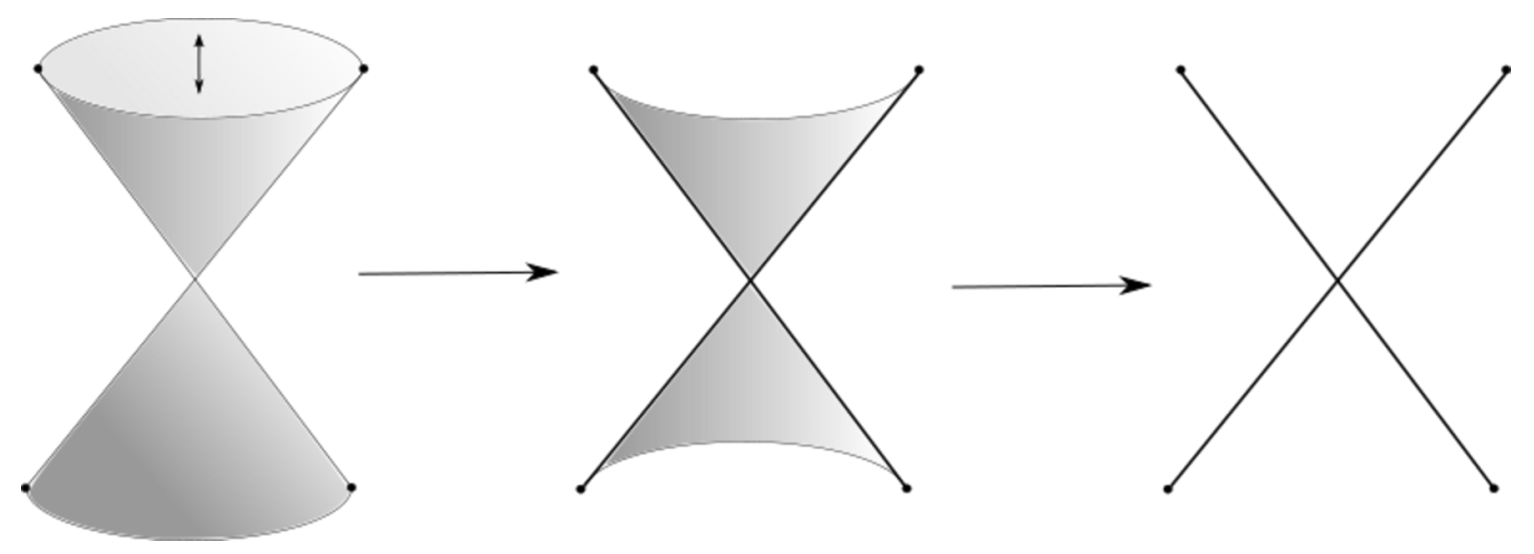

Figure 2. (left) Initial Coulomb branch with highlighted $\mathbb{Z}_{2}$ symmetry. (middle) Coulomb branch of the discretely gauged quiver depicted as an orbifold of the original space. Note that bold edges form a singular subspace under the $\mathbb{Z}_{2}$ symmetry. (right) Coulomb branch of the folded quiver, the subspace fixed under the $\mathbb{Z}_{2}$ symmetry.

for an algebraic group $G$ (the subvariety of the affine Grassmannian corresponding to the so-called small coweights of $G$; see [15] for a friendly introduction addressed to physicists) possess a $\mathbb{Z}_{2}$ global involution - here these orbits would be depicted as the left portion of figure 2. Some of these orbits can be mapped to so-called Reeder pieces which are the union of two nilpotent orbits of $G$, one which can be identified with a $\mathbb{Z}_{2}$ quotient of the affine Grassmannian slice, and the other as the $\mathbb{Z}_{2}$ fixed points - respectively the middle and right parts of figure 2. Coulomb branches of framed unitary ADE quivers were identified with slices in the associated affine Grassmannian [16], following the construction [17, 18], and the $\mathbb{Z}_{2}$ involution of [14] is realized on the quiver as leg permutations like in figure 1. As a consequence, several of the examples discussed below follow from the geometric point of view from these previous works; the present paper sheds a new light on this topic by providing quivers for each of the three spaces, and giving formulas to compute the Hilbert series and HWGs of their closures.

Table 1 features a third discrete action called crossing. Flavorless simply laced quiver theories possess a certain freedom of reparametrisation: the gauge group $G$ factorises as $G / \mathrm{U}(1) \times \mathrm{U}(1)$, with the decoupled $\mathrm{U}(1)$ factor contributing a (geometrically uninteresting) factor of $\mathbb{R}^{3} \times S^{1}$ to the Coulomb branch, which is discarded by convention. Crucially, while the choice of $\mathrm{U}(1)$ is somewhat constrained, the allowed options are in practice equivalent and one can in particular choose to ungauge any given $U(1)$ node without affecting the Coulomb branch. The situation is modified for non-simply laced quivers, where ungaugings on opposite sides of the directed multiple link give rise to pairs of Coulomb branches where one is the discrete quotient of the other. We list this case for the sake of completeness, but do not study it further in this paper. The reader could instead consult the recent treatment in [19]. 


\begin{tabular}{|c|c|c|c|c|}
\hline $\begin{array}{c}\text { Operation } \\
\text { on gauge } \\
\text { theory }\end{array}$ & Quiver description & $\begin{array}{l}\text { Resulting } \\
\text { quiver }\end{array}$ & $\begin{array}{c}\text { String } \\
\text { background } \\
\text { action }\end{array}$ & $\begin{array}{c}\text { Operation on } \\
\text { Coulomb branch }\end{array}$ \\
\hline $\begin{array}{l}\text { Discrete } \\
\text { gauging }\end{array}$ & $b \quad \underset{a}{\bigcirc} \quad \underset{\sim}{\bigcirc} S_{2}$ & $\begin{array}{l}\text { Wreathed } \\
\text { quiver }\end{array}$ & Orbifold & $\begin{array}{c}\text { Discrete quotient } \\
\text { (dimension } \\
\text { preserving) }\end{array}$ \\
\hline Folding & $\bigcirc_{b} \rightarrow{ }_{a} \Rightarrow$ & $\begin{array}{l}\text { Non-simply } \\
\text { laced quiver }\end{array}$ & Orientifold & $\begin{array}{l}\text { Fixed points } \\
\text { (not dimension } \\
\text { preserving) }\end{array}$ \\
\hline Crossing & 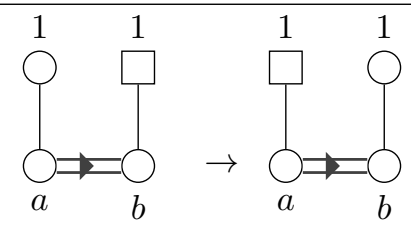 & $\begin{array}{l}\text { Non-simply } \\
\text { laced quiver }\end{array}$ & $?$ & $\begin{array}{c}\text { Discrete quotient } \\
\text { (dimension } \\
\text { preserving) }\end{array}$ \\
\hline
\end{tabular}

Table 1. Discrete actions on the quiver.

Kostant-Brylinski reductions. In [20] the authors identified that discrete quotients of certain minimal nilpotent orbits were equivalent to (generically non-minimal) nilpotent orbits of other algebras; their results are summarised in table $2 .^{4}$ The same pattern is observed in discrete gauging and we claim that our construction is a physical realisation of their cases 1,2,3,4 and 9. We empirically confirmed this conjecture using both Hilbert series and abelianisation methods as in [7] up to low but non-trivial rank. The lines painted in green (cases 2, 3, 4 and 9) correspond to wreathed simply laced quivers. Case 1, painted in red, stands apart because of the non-simply laced initial quiver; although the moduli space can be described algebraically using abelianised variables, the explicit implementation of the monopole formula for non-simply laced wreathed quivers is postponed for future investigations.

A recent work [19] showed that cases 5, 6 and 7 (yellow in table 2) occur in Coulomb branches of non-simply laced quivers. The $\mathbb{Z}_{n}$ quotient corresponds to gauging a U(1) node on the "long" end of an edge of multiplicity $n$ and ungauging another U(1) node on the "short" end. The quiver realisations of all eight known cases are collected in table 2.

Case number 8 still presents a challenge, and we are not aware of any quiver realisation of the corresponding $\mathbb{Z}_{2}^{2}$ quotient. However the HWGs are under control, and are discussed briefly at the end of section 3.5.

\footnotetext{
${ }^{4}$ Ref. [21] provide more examples of discrete and non-discrete quotients in nilpotent orbits.
} 


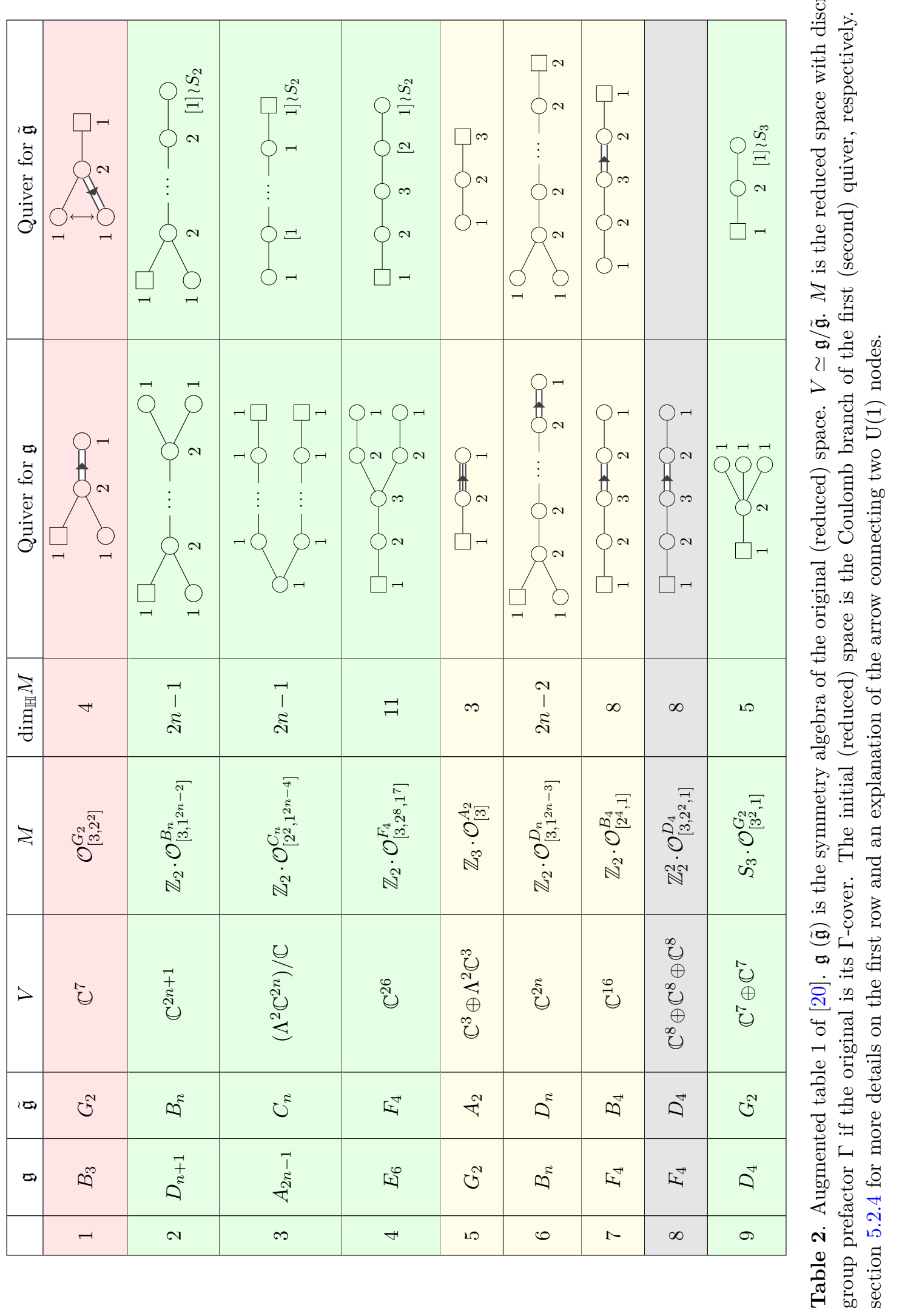




\section{Preliminaries}

\subsection{Folding of Dynkin diagrams}

Some pairs of simple Lie algebras can be related by an operation called folding [22], which acts on an algebra's Dynkin diagram and its internal structure. In a prototypical example, the $D_{4}$ algebra folds into $B_{3}$; in other words, rotations in eight dimensions are restricted to seven. Moreover, we show the $D_{4}$ Chevalley-Serre basis folds to its $B_{3}$ counterpart.

For simplicity, ${ }^{5}$ let $\mathfrak{g}$ be a complex simple Lie algebra associated to a Dynkin diagram of type $A_{2 n-1}, D_{n+1}$ or $E_{6}$. $\mathfrak{g}$ has a (up to some choices of sign) canonical Chevalley-Serre basis obeying

$$
\begin{aligned}
{\left[H_{a}, H_{b}\right] } & =0 \\
{\left[H_{a}, E_{ \pm i}\right] } & = \pm \kappa_{i a} E_{ \pm i} \\
{\left[E_{i}, E_{-i}\right] } & =H_{i} \\
{\left[E_{ \pm i}, \cdot\right]^{1-\kappa_{j i}} E_{ \pm j} } & =0 .
\end{aligned}
$$

where $H_{a}$ span the Cartan subalgebra, $E_{ \pm i}$ are step operators and the indices $1 \leq i, a \leq$ $\operatorname{rank}(\mathfrak{g})$ range over nodes of the diagram as in table 3. $\kappa$ is the Cartan matrix. Throughout this work we will often use a matrix realisation of the Chevalley-Serre basis, in which case we follow the construction of [7].

Dynkin diagrams can be folded if there is a graph automorphism such that no node is linked to its own image under the automorphism. In particular, the diagrams for $A_{2 n-1}$, $D_{n}$ or $E_{6}$ satisfy this constraint as they possess $S_{2}$ graph automorphisms, while the special case $D_{4}$ is invariant under $S_{3}$. In a unique case, $B_{3}$ folds to $G_{2}$ despite lacking an obvious graph automorphism (see figure 30.14 in [22]). The associated algebra $\mathfrak{g}$ is then folded to $\tilde{\mathfrak{g}}$ by the following recipe.

First let us denote the set of automorphisms by $\Gamma$, which is in practice either $S_{2}$ or $S_{3}$, and its elements by $\pi \in \Gamma$. We write

$$
\pi(i)=j
$$

to express that under the automorphism $\pi$ the $i$-th node is mapped to the $j$-th node. The fact that $\pi$ is a Dynkin diagram automorphism translates into the following invariance of the Cartan matrix under the action of $\pi: \kappa_{\pi(i) \pi(a)}=\kappa_{i a}$.

We define the folding function $f$ taking as input nodes of the unfolded Dynkin diagram and mapping them to appropriate nodes in the folded diagram. Consequently, $f \circ \pi=f$. As an example, take $A_{2 n-1}$ which folds to $C_{n}$ and think of $f$ as acting on indices $i$ of the original linear diagram. $f$ acts as $f(1)=f(2 n-1)=1, f(2)=f(2 n-2)=2$ and so on, but $f(n)=n$.

\footnotetext{
${ }^{5}$ Results for semi-simple Lie algebras follow the same pattern.
} 


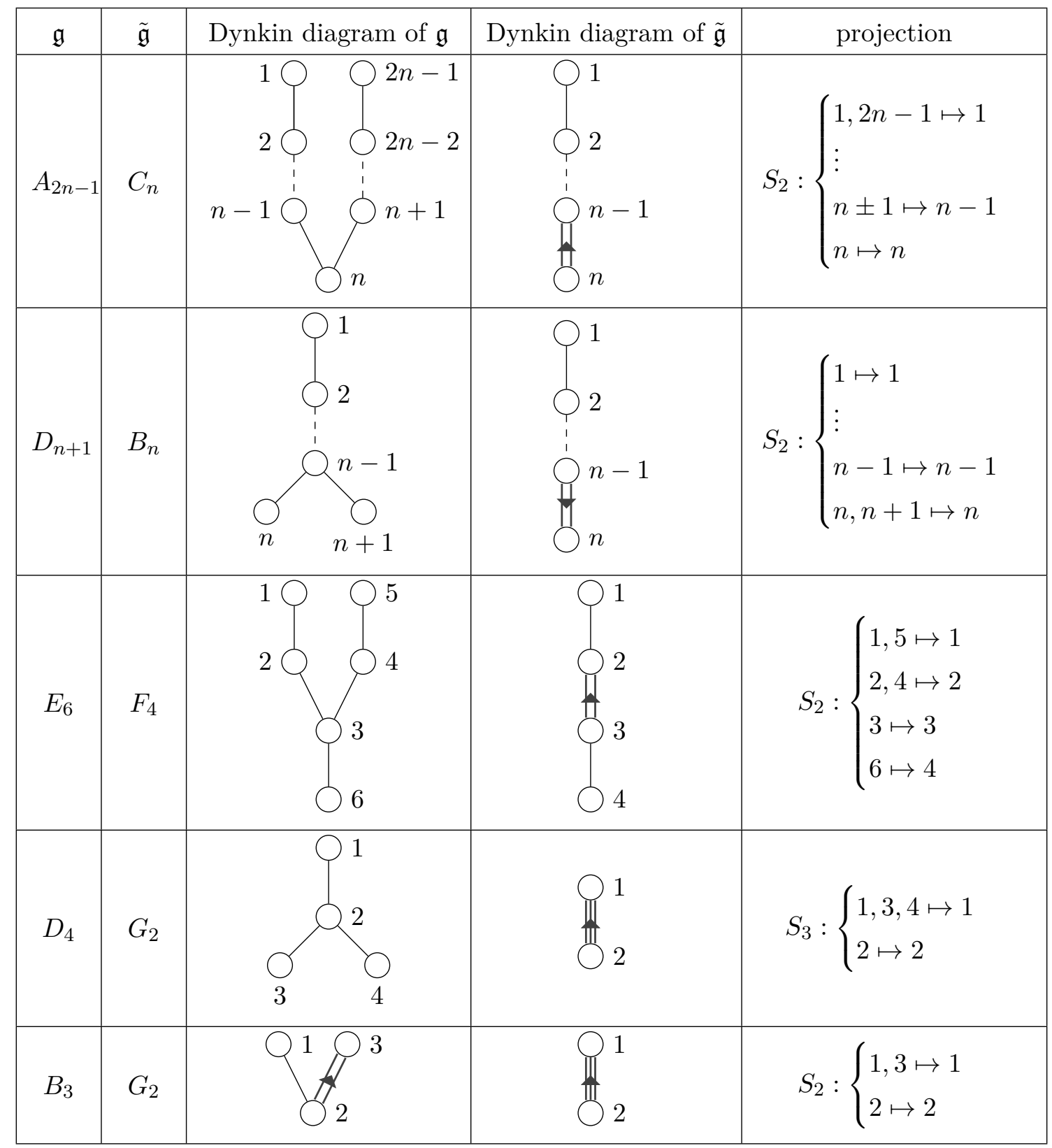

Table 3. Foldable simple Lie algebras. Note that numbers label nodes and do not indicate gauge groups as these are not quiver theories. The $S_{2}$ last row is a special case treated in several places in the main text. 
The folding procedure is now easily stated:

$$
\begin{aligned}
\tilde{H}_{a} & =\sum_{b: f(b)=a} H_{b} \\
\tilde{E}_{ \pm i} & =\sum_{j: f(j)=i} E_{ \pm j}
\end{aligned}
$$

This defines the Chevalley-Serre basis for the folded algebra $\tilde{\mathfrak{g}}$. In the case of $A_{2 n-1}$, the folded algebra is indeed $C_{n}$.

Special care must be taken when folding non-simple roots. Sometimes a sign change is required to preserve the algebra homomorphism $\mathfrak{g} \rightarrow f(\mathfrak{g})$. Consider the case of $A_{3} \rightarrow C_{2}$. $A_{3}$ includes two elements $E_{12}=-\left[E_{1}, E_{2}\right]$ and $E_{23}=-\left[E_{2}, E_{3}\right]$. According to the definition of folding just given, $\tilde{E}_{1}=E_{1}+E_{3}$ and $\tilde{E}_{2}=E_{2}$. Then it follows that

$$
\tilde{E}_{12}=-\left[\tilde{E}_{1}, \tilde{E}_{2}\right]=-\left[E_{1}+E_{3}, E_{2}\right]=-\left(\left[E_{1}, E_{2}\right]+\left[E_{3}, E_{2}\right]\right)=E_{12}-E_{23} .
$$

In this specific case it is clear that the sign flips because the third node, which comes after the second, is mapped to the first, which comes before the second. Likewise it is clear that such a scenario will never occur in the case $D_{n+1} \rightarrow B_{n}$ and only comes into play for $A_{2 n-1} \rightarrow C_{n}, B_{3} \rightarrow G_{2}, D_{4} \rightarrow G_{2}$ and $E_{6} \rightarrow F_{4}$.

The interested reader can easily check (2.1)-(2.3) and (2.4) with a bit more effort. To illustrate the typical calculation, we will confirm (2.2) for $A_{5}$ folding to $C_{3}$. The Cartan matrix of $C_{3}$ is

$$
\kappa=\left(\begin{array}{ccc}
2 & -1 & 0 \\
-1 & 2 & -1 \\
0 & -2 & 2
\end{array}\right)
$$

and

$$
\begin{aligned}
& {\left[\tilde{H}_{2}, \tilde{E}_{3}\right]=\left[H_{2}+H_{4}, E_{3}\right]=-E_{3}-E_{3}=-2 \tilde{E}_{3}=\kappa_{32} \tilde{E}_{3}} \\
& {\left[\tilde{H}_{3}, \tilde{E}_{2}\right]=\left[H_{3}, E_{2}+E_{4}\right]=-E_{2}-E_{4}=-\tilde{E}_{2}=\kappa_{23} \tilde{E}_{2}}
\end{aligned}
$$

Folded Lie algebras sometimes preserve additional tensors. In the case of $C_{n}$ there exists a tensor $J$ such that for every $X$ in $C_{n}$

$$
X^{T} J+J X=0 .
$$

We can also reverse this statement: every $X$ in $A_{2 n-1}$ which satisfies (2.11) is in $C_{n}$.

In our convention $J$ assumes the following form:

$$
J=\left(\begin{array}{ccccccc}
0 & 0 & 0 & \ldots & 0 & 0 & 1 \\
0 & 0 & 0 & \ldots & 0 & -1 & 0 \\
0 & 0 & 0 & \ldots & 1 & 0 & 0 \\
\vdots & \vdots & \vdots & \vdots & \vdots & \vdots & \vdots \\
0 & 0 & -1 & \ldots & 0 & 0 & 0 \\
0 & 1 & 0 & \ldots & 0 & 0 & 0 \\
-1 & 0 & 0 & \ldots & 0 & 0 & 0
\end{array}\right)
$$


The other case of this type is $G_{2}$, which is the subalgebra of $\mathrm{SO}(7)$ preserving the following rank 3 antisymmetric tensor $\phi$ :

$$
\sum_{a^{\prime}} \phi_{a^{\prime} b c} X_{a^{\prime} a}+\sum_{b^{\prime}} \phi_{a b^{\prime} c} X_{b^{\prime} b}+\sum_{c^{\prime}} \phi_{a b c^{\prime}} X_{c^{\prime} c}=0
$$

for all $X \in G_{2}$. Given our choice of Chevalley-Serre basis the tensor is defined as

$$
\phi_{127}=-\phi_{136}=-\phi_{145}=\phi_{235}=-\phi_{246}=-\phi_{347}=-\phi_{567}=1
$$

with the remaining values either fixed by antisymmetry or equal to 0 .

The dual Chevalley-Serre basis of linear forms $\left\{X_{i}^{*}\right\}$ is defined to obey $X_{i}^{*}\left(X_{j}\right)=\delta_{i j}$ for all $X_{i}$ in the Chevalley-Serre basis. In practice we realise $X_{i}^{*}$ as square matrices of the same dimension as $X_{i}$ and represent the evaluation as the linear extension of

$$
X_{i}^{*}\left(X_{j}\right)=\left\langle X_{i}^{*}, X_{j}\right\rangle=\left\langle X_{j}, X_{i}^{*}\right\rangle=\operatorname{tr}\left(X_{i}^{*} X_{j}\right) .
$$

The dual Chevalley-Serre bases of "parent" and folded algebras are related:

$$
\begin{aligned}
\tilde{H}_{a}^{*} & =\frac{1}{\#_{a}} \sum_{b: f(b)=a} H_{b}^{*} \\
\tilde{E}_{ \pm i}^{*} & =\frac{1}{\#_{i}} \sum_{j: f(j)=i} E_{ \pm j}^{*}
\end{aligned}
$$

where $\#_{i}$ denotes the multiplicity of node $i$ defined as

$$
\# i=|\{j: f(j)=i\}|
$$

For example:

$$
\left\langle\tilde{H}_{a}, \tilde{H}_{b}^{*}\right\rangle=\frac{1}{\#_{b}} \sum_{\begin{array}{c}
c: f(c)=a \\
d: f(d)=b
\end{array}}\left\langle H_{c}, H_{d}^{*}\right\rangle=\frac{1}{\#_{b}} \sum_{\begin{array}{r}
c: f(c)=a \\
d: f(d)=b
\end{array}} \delta_{c d}=\frac{1}{\#_{b}} \#_{b} \delta_{a b}=\delta_{a b}
$$

where the second-to-last equality follows from the fact that $c=d$ can only occur if both fold to the same node, ie. $a=b$, and that this can happen for $\#_{b}$ joint choices of (identical) $c$ and $d$.

We close this section with a brief discussion of the aforementioned case of $B_{3}$ folding to $G_{2}$ despite a lack of graph automorphisms. This is easily elucidated with a quick detour through $D_{4}$ :

$$
\begin{aligned}
\tilde{H}_{3}^{B} & =H_{3}^{D}+H_{4}^{D} \\
\tilde{H}_{2}^{G} & =H_{1}^{D}+H_{3}^{D}+H_{4}^{D}=\tilde{H}_{1}^{B}+\tilde{H}_{3}^{B}
\end{aligned}
$$

where we decorate each Cartan generator with a subscript denoting its algebra. As illustrated - and the pattern holds up for remaining $G_{2}$ basis elements - $G_{2}$ can be expressed as a folding of $B_{3}$ in the same way that $B_{3}$ is a folding of $D_{4}$. 


\subsection{The monopole formula}

The monopole formula is an important computational tool in the study of Coulomb branch chiral rings which was introduced and subsequently generalised in [1, 2]. The formula calculates the chiral ring Hilbert series by counting monopole operators labelled by their conformal dimension and, optionally, action under $\mathrm{U}(1)$ global symmetries arising from unitary gauge nodes.

Consider a simply laced quiver. The underlying graph is made of a set of vertices $V$ and a set of (unoriented) edges $E \subset S^{2}(V)$. To each vertex $v \in V$ is associated a gauge group $\mathrm{U}\left(n_{v}\right)$, and to each edge $e \in E$ is associated a hypermultiplet in the bifundamental of $\mathrm{U}\left(n_{v}\right) \times \mathrm{U}\left(n_{v^{\prime}}\right)$ where $e=\left(v, v^{\prime}\right)$. Finally, we have a set of flavor vertices $F \neq \emptyset$ with global symmetries $\mathrm{SU}\left(n_{f}\right)$ for $f \in F$, and a set of edges $E^{\prime} \subset V \times F$. An edge $e^{\prime}=(v, f)$ stands for $n_{f}$ hypermultiplets in the fundamental representation of $\mathrm{U}\left(n_{v}\right)$. The total gauge group is

$$
G=\prod_{v \in V} \mathrm{U}\left(n_{v}\right)
$$

and it has rank

$$
r=\sum_{v \in V} n_{v}
$$

The Weyl group is

$$
W=\prod_{v \in V} S_{n_{v}} .
$$

A magnetic charge is an element $m \in \mathbb{Z}^{r}$. For $H$ a subgroup of $S_{r}$ and $m$ a magnetic charge, we define the stabiliser

$$
H(m)=\{g \in H \mid g \cdot m=m\}
$$

The conformal dimension $\Delta(m)$ is defined by

$$
2 \Delta(m)=\sum_{\left(v, v^{\prime}\right) \in E} \sum_{i=1}^{n_{v}} \sum_{i^{\prime}=1}^{n_{v^{\prime}}}\left|m_{v, i}-m_{v^{\prime}, i^{\prime}}\right|+\sum_{(v, f) \in E^{\prime}} \sum_{i=1}^{n_{v}} n_{f}\left|m_{v, i}\right|-\sum_{v \in V} \sum_{i=1}^{n_{v}} \sum_{j=1}^{n_{v}}\left|m_{v, i}-m_{v, j}\right| .
$$

Then the (unrefined) Hilbert series for the Coulomb branch of the quiver is given by the monopole formula, which can be written as

$$
\operatorname{HS}(t)=\operatorname{HS}_{W}(t)=\frac{1}{|W|} \sum_{m \in \mathbb{Z}^{r}} \sum_{\gamma \in W(m)} \frac{t^{2 \Delta(m)}}{\operatorname{det}\left(1-t^{2} \gamma\right)}
$$

where $W$ is the Weyl group (2.22). The form of this formula slightly differs from the one presented in [2]; in particular note the absence of the Casimir symmetry factors and the summation on the whole magnetic lattice. One can show that despite these superficial differences the formulas are equivalent. The form (2.25) is better suited for generalisation to wreathed quivers in section 3. 
This formula can be further refined by labelling each monopole insertion with its charge under the topological symmetry $q_{v}$. We only need introduce $|V|$ extra fugacities $z_{v}$ :

$$
\operatorname{HS}_{\mathrm{ref}}\left(t, z_{v}\right)=\operatorname{HS}_{\mathrm{ref}, W}\left(t, z_{v}\right)=\frac{1}{|W|} \sum_{m \in \mathbb{Z}^{r}} \sum_{\gamma \in W(m)} \frac{\left(\prod_{v} z_{v}^{q_{v}(m)}\right) t^{2 \Delta(m)}}{\operatorname{det}\left(1-t^{2} \gamma\right)} .
$$

Reference [1] further improved the formula with the addition of non-simply laced quivers to the world of quiver gauge theories. While they were not explicitly constructed (say, as Lagrangian theories), it was relatively straightforward to modify the monopole formula such that, when computed for non-simply laced quivers, the results made sense and followed the pattern of their simply-laced cousins. In particular, it is well known that balanced quivers' Coulomb branch symmetry enhances according to the Dynkin diagram which the quiver resembles. For example, balanced linear quivers exhibit $A_{n}$ symmetry. Non-simply laced balanced quivers were found to have $B_{n}, C_{n}, F_{4}$ or $G_{2}$ symmetry.

The only difference introduced by non-simply laced quivers to the monopole formula is a modification of (2.24) to

$$
\begin{aligned}
2 \Delta(m)= & \sum_{\left(v, v^{\prime}\right) \in E} \sum_{i=1}^{n_{v}} \sum_{i^{\prime}=1}^{n_{v^{\prime}}}\left|\kappa_{v, v^{\prime}} m_{v, i}-\kappa_{v^{\prime}, v} m_{v^{\prime}, i^{\prime}}\right|+\sum_{(v, f) \in E^{\prime}} \sum_{i=1}^{n_{v}} n_{f}\left|m_{v, i}\right| \\
& -\sum_{v \in V} \sum_{i=1}^{n_{v}} \sum_{j=1}^{n_{v}}\left|m_{v, i}-m_{v, j}\right|
\end{aligned}
$$

where $\kappa$ is defined as follows:

- $\kappa_{v v}=2$

- $\kappa_{v v^{\prime}}=\kappa_{v^{\prime} v}=-n$ if $v$ and $v^{\prime}$ are connected by $n$ undirected edges

- $\kappa_{v v^{\prime}}=-n, \kappa_{v^{\prime} v}=-1$ if $v$ and $v^{\prime}$ are connected by an $n$-valent directed edge from $v$ to $v^{\prime}$

The similarity to Cartan matrices is, of course, not coincidental and reappears in the abelianised formalism.

Reading relations off the Hilbert series. We briefly describe the method by which we extract chiral ring relations from the Coulomb branch Hilbert series. Assume that the Hilbert series is refined with fugacities $z_{i}$ counting charge under a Cartan subalgebra of the Coulomb branch symmetry algebra $\mathfrak{g}$. The Hilbert series expands as

$$
\operatorname{HS}\left(t, z_{i}\right)=\sum_{s \in \mathbb{Z} \geq 0} p_{s}\left(z_{i}\right) t^{s}
$$

where $p_{s}\left(z_{i}\right)$ are characters of $\mathfrak{g}$.

We first state the general strategy for a nilpotent orbit, whose coordinate ring is generated by a single (co)adjoint representation with $R$-symmetry spin 1 . The quaternionic dimension of each Coulomb branch is easily calculated by summing up gauge ranks, which 
is unaffected by discrete gauging. Knowing the dimension and global symmetry, we can look up the space in [23]. ${ }^{6}$

We could then expand the highest weight generating function, comparing (polynomial) coefficients of $t^{2 n}$ to the character representation of the $n$-th symmetric product $\operatorname{Sym}{ }^{n} \operatorname{adj}(\mathfrak{g})$ and find missing representations suggesting the existence of relations. Or we can perform the same computation in a more elegant fashion using the plethystic logarithm:

$$
\mathrm{PL}(\mathrm{HS})\left(t, z_{i}\right)=\sum_{k=1}^{\infty} \frac{\mu(k)}{k} \log \left(\mathrm{HS}\left(t^{k}, z_{i}^{k}\right)\right)=\sum_{s=1}^{\infty} g_{s}\left(z_{i}\right) t^{s}-\sum_{s=1}^{\infty} r_{s}\left(z_{i}\right) t^{s}
$$

where $\mu(k)$ is the Möbius function and $g_{s}\left(z_{i}\right)$ and $r_{s}\left(z_{i}\right)$ are characters of $\mathfrak{g}$. If the space is a complete intersection, the list of $g_{s}$ and $r_{s}$ is finite and they correspond respectively to the generators and relations of the Coulomb branch. The minimal set of relations is typically present in the first few orders of $t$. For example, the (closure of the) minimal nilpotent orbit of any simple algebra $\mathfrak{g}$ (whose coordinate ring is generated by one coadjoint generator [24]) is described by a set of Joseph relations $[25,26]$ of its coordinate ring. They are always necessarily quadratic in the coadjoint generator. In more general cases we go to slightly higher order, $t^{6}$ or $t^{8}$. Then, whenever feasible, we verify that the full set of relations are identified: we calculate the Hilbert series of a ring defined by $\operatorname{dim} \mathfrak{g}$ generators subject to the relations in question and compare it to tabulated expressions.

This procedure is only slightly modified in the few isolated cases in this paper where the Coulomb branch is not a nilpotent orbit. The chiral ring is then generated by more generators, which are in these particular cases also coadjoint. Their contribution will be visible in the PL.

\subsection{Abelianisation}

Coulomb branch chiral rings of simply-laced unitary quiver theories can be explicitly constructed following the prescriptions of $[4,27,28]$. One first defines the abelianised ring and then reduces it to a particular Weyl-invariant sub-ring.

Let $i$ index the vertices and hence gauge group factors of a quiver gauge theory. Each gauge node $G_{i}$ contributes several basic variables to the ring: $u_{i, a}^{+}, u_{i, a}^{-}$and $\varphi_{i, a}$, where $1 \leq a \leq \operatorname{rank} G_{i}$. We will sometimes blur the distinction between the three types of variables by dropping all identifying information except for the node and gauge indices, leaving only $x_{i, a}$. The variables satisfy abelianised relations

$$
u_{i, a}^{+} u_{i, a}^{-}=-\frac{\prod_{w \in \mathcal{R}}\left\langle w,\left.\vec{\varphi}\right|^{\left|w_{i, a}\right|}\right.}{\prod_{\alpha \in \Phi}\langle\alpha, \vec{\varphi}\rangle^{\left|\alpha_{i, a}\right|}}
$$

where $\vec{\varphi}=\left(\varphi_{1,1}, \ldots, \varphi_{n, \text { rank } G_{n}, M_{1,1}, \ldots M_{n, N_{f}^{n}}}\right), N_{f}^{i}$ is the number of fundamental flavors on the $i$-th node, and both the roots $\alpha$ and weights $w$ are expressed as weights in the weight basis of the theory's gauge group $G=\prod_{i=1}^{n} G_{i}$.

\footnotetext{
${ }^{6}$ This paper differs from the present paper in the simple root convention for $G_{2}$ : for the present paper the (co)adjoint representation goes by [01] whereas in [23] the two labels are swapped.
} 
For example $\mathrm{U}(2)$ with 4 fundamental flavors comes with the following matter and gauge representations:

$$
\mathcal{R}=\{(1,0 ;-1,0,0,0), \ldots(1,0 ; 0,0,0,-1),(0,1 ;-1,0,0,0), \ldots(0,1 ; 0,0,0,-1)\}
$$

with the first two charges belonging to $\mathrm{U}(2)$ and the last four belonging to $\mathrm{SU}(4)$ and

$$
\Phi=\{(1,-1),(-1,1)\}
$$

where the charges are associated to $\mathrm{U}(2)$.

The Coulomb branch is a symplectic space and its chiral ring carries a Poisson bracket, which descends from a bracket defined on the abelianised ring:

$$
\begin{aligned}
& \left\{\varphi_{i, a}, u_{i, a}^{ \pm}\right\}= \pm u_{i, a}^{ \pm} \\
& \left\{u_{i, a}^{+}, u_{i, a}^{-}\right\}=\frac{\partial}{\partial \varphi_{i, a}} \frac{\prod_{w \in \mathcal{R}}\langle w, \vec{\varphi}\rangle^{\left|w_{i, a}\right|}}{\prod_{\alpha \in \Phi}\langle\alpha, \vec{\varphi}\rangle^{\left|\alpha_{i, a}\right|}} \\
& \left\{u_{i, a}^{ \pm}, u_{j, b}^{ \pm}\right\}= \begin{cases} \pm \kappa_{i j} \frac{u_{i, a}^{ \pm} u_{j, b}^{ \pm}}{\varphi_{i, a}-\varphi_{j, b}} & \text { if }(i, a) \neq(j, b) \\
0 & \text { if }(i, a)=(j, b)\end{cases}
\end{aligned}
$$

where $\kappa_{i j}$ is defined as in section 2.2. (The remaining Poisson brackets vanish.)

These Poisson brackets, along with the abelianised relations (2.30), generate every element of the "abelianised" chiral ring $\mathbb{C}\left[\mathcal{C}_{\text {abel }}\right]$. The true Coulomb branch chiral ring $\mathbb{C}[\mathcal{C}]$ is a sub-ring of the Weyl-symmetrised ring $\mathbb{C}\left[\mathcal{C}_{\text {abel }}\right]^{W_{G}}$. We leverage information from the Hilbert series to identify the correct subring, picking Weyl-invariant abelianised operators with the correct representation-theoretic properties. For more details on the abelianised ring, and a specific example, see appendix A.

\subsection{Construction of Coulomb branch multiplets}

Abelianised variables $\varphi_{i, a}$ have weight 2 under the $R$-symmetry ${ }^{7}$ while the Poisson bracket scales with weight -2 . Weights of $u_{i, a}^{ \pm}$can be read off from (2.30). The Coulomb branch chiral ring of any good or ugly theory is graded by $R$-symmetry weights as $\mathbb{C}[\mathcal{C}]=\sum_{i \in \mathbb{Z}>0} \mathbb{C}[\mathcal{C}]_{i}$ where $\mathbb{C}[\mathcal{C}]_{i}$ is the vector space of all Coulomb branch chiral ring operators with $R$ symmetry weight $i$.

Any Coulomb branch operator $\mathcal{O}$ with well-defined $R$-symmetry weight $j$ defines a map $\{\mathcal{O}, \cdot\}: \mathbb{C}[\mathcal{C}]_{i} \rightarrow \mathbb{C}[\mathcal{C}]_{i+j-2}$ and therefore operators in $\mathbb{C}[\mathcal{C}]_{2}$ form a closed Poisson algebra. This algebra is precisely the symmetry algebra $\mathfrak{g}$ of the Coulomb branch and all operators in $\mathbb{C}[\mathcal{C}]_{i}$ necessarily assemble into tensors of the $\mathbb{C}[\mathcal{C}]_{2}$ algebra $\mathfrak{g}$. In this paper we focus almost exclusively on good (in fact, balanced) theories whose Coulomb branch chiral rings are generated by operators in $\mathbb{C}[\mathcal{C}]_{2}$ and whose symmetry algebra $\mathfrak{g}$ is simple. Consequently, $\mathbb{C}[\mathcal{C}]_{2}$ operators assemble into a single (coadjoint) representation of $\mathfrak{g}$ - the moment map of the symmetry - which has a matrix realisation for all cases in this article.

\footnotetext{
${ }^{7}$ The $R$-symmetry is assumed to be the $\mathrm{SU}(2)$ factor acting non-trivially on the Coulomb branch. An operator's weight is twice its conformal dimension.
} 
We will also consider two cases (one in the main text, one in an appendix) in which the ring is generated by $\mathbb{C}[\mathcal{C}]_{2}$ operators transforming in the coadjoint representation along with another set of $\mathbb{C}[\mathcal{C}]_{4}$ operators, also in the coadjoint representation; the following discussion straightforwardly generalises for such cases.

In [7] two of this paper's authors developed a prescription for coadjoint chiral ring generators following [4]. Let $X_{k} \in \mathfrak{g}$ form a basis of $\mathfrak{g}$ satisfying $\left[X_{k}, X_{l}\right]=\sum_{m} c_{k l m} X_{m}$. There is a basis of $\mathbb{C}[\mathcal{C}]_{2}$ formed by $\mathcal{O}_{k}$ such that $\left\{\mathcal{O}_{k}, \mathcal{O}_{l}\right\}=\sum_{m} c_{k l m} \mathcal{O}_{m}$. If $X_{k}^{*}$ are dual to $X_{k}$, ie. $\left\langle X_{k}^{*}, X_{l}\right\rangle=\delta_{k l}$, the moment map $N^{8}$ is explicitly constructed as

$$
N=\sum_{k} \mathcal{O}_{k} X_{k}^{*}
$$

This definition guarantees that $\langle N, \cdot\rangle$ acts as a Lie algebra homomorphism:

$$
\left\{\left\langle N, X_{k}\right\rangle,\left\langle N, X_{l}\right\rangle\right\}=\left\langle N,\left[X_{k}, X_{l}\right]\right\rangle
$$

The choice of $\mathcal{O}_{k}$ is heavily constrained - enough, in fact, to allow us to select an almost unique $^{9}$ set of operators from $\mathbb{C}\left[\mathcal{C}_{\text {abel }}\right]^{W_{G}}$ to form components of $N$. And since $N$ generates the Coulomb branch chiral ring, we have found a set of generators for $\mathbb{C}[\mathcal{C}]$.

The moment map $N$ satisfies certain matrix relations, which can be inferred from the Hilbert series of the Coulomb branch. Let us denote the ideal they form as $I_{M}$, and let $I_{A}$ stand for the ideal of abelian relations (2.30). Then we claim that

$$
\mathbb{C}[\mathcal{C}]=\mathbb{C}\left[N\left(u_{i, a}^{ \pm}, \varphi_{i, a}\right)\right] / I_{A}=\mathbb{C}[N] / I_{M}
$$

Note that the third object contains no abelian expressions (and hence no potentially troubling factors of $\left.1 /\left(\varphi_{i}-\varphi_{j}\right)\right)$. In this last step abelianised relations and components of $N$, both expressed in terms of abelianised variables $u^{ \pm}$and $\varphi$, are replaced by symbolic components of $N$ (ie. $N_{i j}$ ) with matrix relations also expressed in terms of components of $N$.

It is in effect a change of variables to a set which is well-defined even on the nonabelian locus of the moduli space. On the other hand, the less-than-perfect abelianised representation brings with it a major advantage: it is very explicit and rigid. We can use it to construct the generator $N$, independently check its relations and also calculate exact coefficients in these relations (including dependence on mass parameters) - something which is forever off-limits to any method relying solely on Hilbert series, all while keeping representation theory front and centre.

This combined approach is called the synthetic method in [7]. We construct moment maps for all examples in this paper and explicitly check the expected relations. By finding total agreement between predictions of Hilbert series and our explicit construction of folded quivers, we will argue for specific interpretations of the multiple link from [1] and an operation called discrete gauging (similar to but distinct from that studied in $[5,6]$ ), all at the level of individual and explicitly constructed operators.

\footnotetext{
${ }^{8}$ We reserve the usual symbol for moment maps, $\mu$, for highest weight fugacities.

${ }^{9} \mathrm{It}$ is enough to declare $\sum_{a} u_{i, a}^{+}$a positive simple root operator to fix the remaining choices, at least in the quivers we consider.
} 

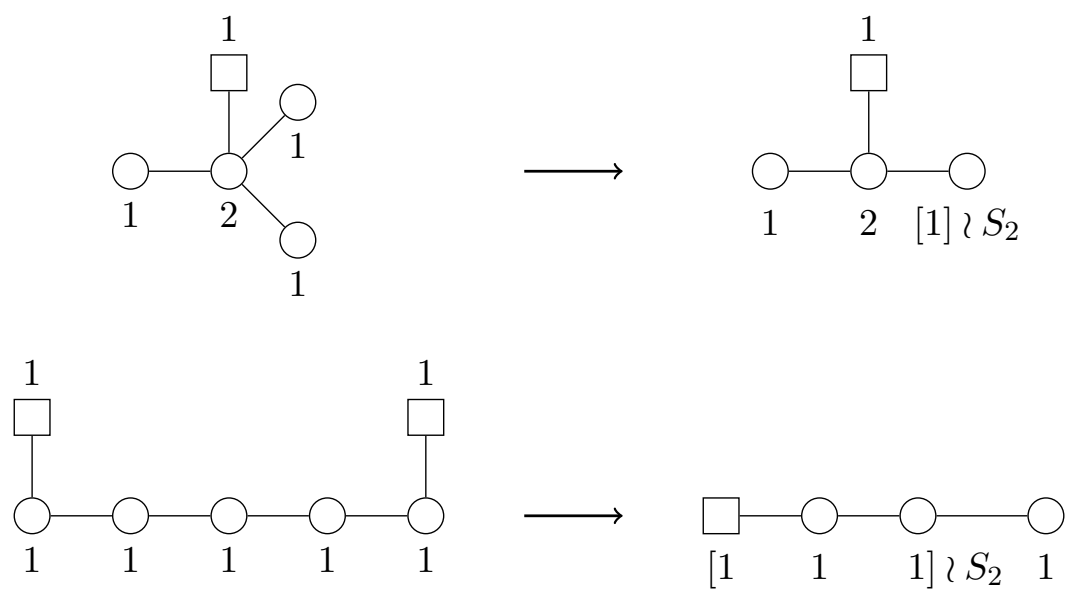

Figure 3. Quivers on the left wreathe into quivers on the right.

\section{Discrete gauging}

Our first example of a discrete quiver operation, discrete gauging, orbifolds the Coulomb branch by a subgroup of the quiver's automorphisms. Another operation, which also acts on the Coulomb branch as an orbifold, was previously studied in $[5,6] .{ }^{10}$ Ours differs in several respects: it preserves the dimension of the Higgs branch as well as the Coulomb branch, allows for consistent and successive discrete gauging of nodes into "larger" nodes and generalises beyond acting on a collection of $n \mathrm{U}(1)$ nodes (which form a $\mathrm{U}(n)$ node with adjoint matter in $[5,6])$ to acting on $n$ copies of arbitrary gauge groups or "legs" of the quiver.

It is possible to discretely gauge any quiver of the type depicted in the top left corner of figure 1 , ie. one with $m$ identical $\operatorname{legs}^{11} Q_{i}$ (and potentially other legs $Q_{j}^{\prime}$ ) connected to a single common node which we call the pivot. ${ }^{12}$ One discretely gauges the $m$ identical legs by extending the overall gauge group with the symmetric group $S_{m}$, or a subgroup thereof, which permutes the gauge factors associated with each leg. We say that we have gauged the quiver's automorphism. For example, three legs composed solely of U(1) nodes will arrange into a $\mathrm{U}(1)\left\{S_{3}\right.$ node, while two legs with $\mathrm{U}(2) \times \mathrm{U}(1)$ gauge nodes will combine to give $(\mathrm{U}(2) \times \mathrm{U}(1))<S_{2}$, with $S_{2}$ simultaneously exchanging $\mathrm{U}(2)$ and $\mathrm{U}(1)$ factors.

Our strategy in this section consists of the following steps. We first demonstrate the existence of a well-defined orbifolding operation on the Coulomb branch, giving results consistent with existing literature. Then we suggest that the operation acts on the quiver as a whole in a way that can be deduced from the Coulomb branch action and that the results of this operation should be viewed as quivers in their own right, even if they often cannot be written down using existing notation; we introduce the concept of wreathed quivers to get over this difficulty; see figure 3 for two examples. We support this claim by

\footnotetext{
${ }^{10}$ In contrast to our treatment, these works did not claim to discretely gauge the theory, but restricted their claims only to effects on the Coulomb branch.

${ }^{11} \mathrm{~A}$ leg can have arbitrary shape and in particular need not be linear.

${ }^{12}$ It may be possible to discretely gauge quivers without a pivot node but we do not have a successful case to present.
} 
generalising the monopole formula to this family of quivers and computing an example, as well as calculating a few wreathed quiver Higgs branches. We also conjecture that a well known Higgs branch operation is the $3 \mathrm{~d}$ mirror to this operation on the Coulomb branch.

\subsection{Wreath product}

We pause for a moment to introduce the notion of the wreath product $G$ 々 $\Gamma$ of a group $G$ by a permutation group $\Gamma \subseteq S_{n}$ (the integer $n$ is understood in the notation $G$ 々 $\Gamma$, which we could denote $G \imath_{n} \Gamma$ if there is a risk of confusion) [29]. As a set, we define

$$
G \imath_{n} \Gamma \equiv G \imath \Gamma=\left(\prod_{i=1}^{n} G_{i}\right) \times \Gamma,
$$

where the $\times$ denotes the Cartesian product of sets, not the direct product of groups. There are $n$ copies $G_{1}, \ldots, G_{n}$ of the group $G$. An element of $(g, \sigma) \in G$ $\Gamma$ is an ordered list of $n$ elements $g_{i}$ of $G$ together with a permutation $\sigma \in \Gamma$. The group multiplication law is given, for $(g, \sigma) \in G \imath \Gamma$ and $\left(g^{\prime}, \sigma^{\prime}\right) \in G \imath \Gamma$, by

$$
(g, \sigma) \cdot\left(g^{\prime}, \sigma^{\prime}\right)=\left(g \sigma\left(g^{\prime}\right), \sigma \sigma^{\prime}\right), \text { with }\left(g \sigma\left(g^{\prime}\right)\right)_{i}=g_{i} g_{\sigma^{-1}(i)}^{\prime} .
$$

Intuitively, $G \nmid \Gamma$ is the direct product of $n$ copies of $G$, which can in addition be permuted by $\Gamma$.

In this paper we consider wreath products where $G$ is a unitary group $\mathrm{U}(r)$, or more generally a direct product of finitely many unitary groups $\mathrm{U}\left(r_{1}\right) \times \cdots \times \mathrm{U}\left(r_{k}\right)$. In this case, in particular in the quivers, we extend the usual shorthand notation in which $\mathrm{U}(r)$ is replaced by the rank $r$, and we write $r \nmid \Gamma$ for $\mathrm{U}(r) \imath \Gamma$, and more generally $\left[\begin{array}{lll}r_{1} & \cdots & r_{k}\end{array}\right] \curlywedge \Gamma$ for $\left(\mathrm{U}\left(r_{1}\right) \times \cdots \times \mathrm{U}\left(r_{k}\right)\right) \imath \Gamma$.

\subsection{Action on the Coulomb branch}

We will first study this procedure through the lens of Coulomb branch abelianisation. The goal is to show that the Coulomb branch can be reduced to an orbifold by an automorphism of the quiver.

Since each node contributes several variables to the abelianised chiral ring, there is an induced $S_{m}$ action permuting them. For any $\pi \in S_{m}$, we have

$$
\pi\left(x_{i, a}\right):=x_{\pi(i), a}
$$

Action on more complicated (polynomial or rational) functions of these variables is defined by action on indices of the full expression. For example

$$
\pi\left(u_{i, a}^{+} u_{j, b}^{+}\right)=u_{\pi(i), a}^{+} u_{\pi(j), b}^{+} .
$$

Note that mass parameters should be treated as numbers (parameters) rather than ring elements (VEVs); therefore $\pi$ does not act on them, ie.

$$
\pi\left(M_{i, a}\right)=M_{i, a}
$$


In fact, this constraint forces

$$
\pi\left(M_{i, a}\right)=M_{i, a}=M_{\pi(i), a} .
$$

To see this consider the $A_{5}$ theory which gauges to the bottom right quiver in figure 3 :

$$
\begin{aligned}
\pi\left(u_{1}^{+} u_{1}^{-}\right) & =\pi\left(-\left(\varphi_{1}-\varphi_{2}\right)\left(\varphi_{1}-M_{1}\right)\right)=-\left(\varphi_{5}-\varphi_{4}\right)\left(\varphi_{5}-M_{1}\right) \\
u_{5}^{+} u_{5}^{-} & =-\left(\varphi_{5}-\varphi_{4}\right)\left(\varphi_{5}-M_{5}\right)
\end{aligned}
$$

Since $\pi\left(u_{1}^{+} u_{1}^{-}\right)=u_{5}^{+} u_{5}^{-}$, the two mass parameters must be equal to preserve symmetry under $\pi$. This is a sensible constraint: if $M_{1} \neq M_{5}$ then the mass deformation breaks the quiver's $S_{2}$ symmetry.

We should check that the form of the Poisson brackets (2.33)-(2.35) is compatible with this action in the sense that $\{\pi(x), \pi(y)\}=\pi(\{x, y\})$.

$$
\begin{aligned}
& \left\{\pi\left(\varphi_{i, a}\right), \pi\left(u_{i, a}^{ \pm}\right\}\right)=\left\{\varphi_{\pi(i), a}, u_{\pi(i), a}^{ \pm}\right\}= \pm u_{\pi(i), a}^{ \pm}=\pi\left(\left\{\varphi_{i, a}, u_{i, a}^{ \pm}\right\}\right) \\
& \left\{\pi\left(u_{i, a}^{+}\right), \pi\left(u_{i, a}^{-}\right)\right\}=\left\{u_{\pi(i), a}^{+}, u_{\pi(i), a}^{-}\right\}=\frac{\partial}{\partial \varphi_{\pi(i), a}} \frac{\prod_{w \in \mathcal{R}}\langle w, \pi(\vec{\varphi})\rangle^{\left|w_{i, a}\right|}}{\prod_{\alpha \in \Phi}\langle\alpha, \pi(\vec{\varphi})\rangle^{2\left|\alpha_{i, a}\right|}}=\pi\left(\left\{u_{i, a}^{+}, u_{i, a}^{-}\right\}\right) \\
& \left\{\pi\left(u_{i, a}^{ \pm}\right), \pi\left(u_{j, b}^{ \pm}\right)\right\}=\left\{u_{\pi(i), a}^{ \pm}, u_{\pi(j), b}^{ \pm}\right\}= \pm \kappa_{i j} \frac{u_{\pi(i), a}^{ \pm} u_{\pi(j), b}^{ \pm}}{\varphi_{\pi(i), a}-\varphi_{\pi(j), b}}=\pi\left(\left\{u_{i, a}^{ \pm}, u_{j, b}^{ \pm}\right\}\right)
\end{aligned}
$$

The first line is clearly compatible with the action. The second line also succeeds with a simple relabelling: $w_{\pi(i)} \leftrightarrow w_{i}$ and $\alpha_{\pi(i)} \leftrightarrow \alpha_{i}$. The third line is similarly preserved because $\kappa_{\pi(i) \pi(j)}=\kappa_{i j}$ is a consequence of the automorphism. In fact, it is noteworthy that the third line forces the action of $\pi$ to preserve connectedness while the second line enforces identical gauge and matter content on each leg $Q_{i}$.

To implement the quotient on the Coulomb branch chiral ring, it is enough to declare that only $S_{m}$-invariant operators are physical. This is easily done through the use of a projector:

$$
P(\cdot)=\frac{1}{m !} \sum_{\pi \in S_{m}} \pi(\cdot)
$$

Every operator of the form $P(\mathcal{O})$ is physical.

The effect on the Coulomb branch is then transparent. If $\mathcal{C}$ and $\tilde{\mathcal{C}}$ are Coulomb branches of, respectively, the original quiver and discretely gauged quivers, the two spaces are related by

$$
\tilde{\mathcal{C}}=\mathcal{C} / S_{m}
$$

ie. the discretely gauged Coulomb branch is a $S_{m}$ orbifold of the original space. This construction leads to new Coulomb branches which were previously unknown, provided that they are orbifolds of known Coulomb branches.

Note that nothing prevents generalisation from $S_{m}$ to arbitrary subgroups $\Gamma$ of $S_{m}$, for instance the alternating group $A_{m}$ or cyclic group $\mathbb{Z}_{m}$. We investigate one such example in section 5.2.5. 
The projector acts remarkably simply on moment maps of type $A D$ quivers as studied in [7]. In fact, if $N_{A_{2 n-1}}$ is the moment map for a type $A_{2 n-1}$ quiver then

$$
P\left(N_{A_{2 n-1}}\right)=N_{C_{n}}
$$

is a $C_{n}$ moment map and $P$ acts component-wise. Similarly,

$$
P\left(N_{D_{n+1}}\right)=N_{B_{n}}
$$

To see this action on an example, and to illustrate why its action on moment maps is so simple, consider the top left quiver in figure 7. Select the Chevalley-Serre basis of $D_{4}$ and its operator counterpart, ie. the basis of operators in $\mathbb{C}[\mathcal{C}]_{2}$ which replicates $(2.1)-(2.4)$ with Poisson brackets. We will denote the algebra elements and their duals with capital letters, reserving lower case letters for operators with appropriate commutation relations. In this notation, the moment map is

$$
N_{D_{4}}=\sum_{1 \leq i \leq 4} h_{i} H_{i}^{*}+\sum_{\alpha \in \Phi^{+}}\left(e_{\alpha} E_{\alpha}^{*}+e_{-\alpha} E_{-\alpha}^{*}\right)
$$

This sum above contains 28 terms, and to exhibit the action of $P$ we show what happens to most of them:

$$
\begin{aligned}
N_{D_{4}}= & h_{1} H_{1}^{*}+h_{2} H_{2}^{*}+h_{3} H_{3}^{*}+h_{4} H_{4}^{*} \\
& +e_{\langle 1,0,0,0\rangle} E_{\langle 1,0,0,0\rangle}^{*}+e_{\langle 0,1,0,0\rangle} E_{\langle 0,1,0,0\rangle}^{*}+e_{\langle 0,0,1,0\rangle} E_{\langle 0,0,1,0\rangle}^{*}+e_{\langle 0,0,0,1\rangle} E_{\langle 0,0,0,1\rangle}^{*} \\
& +e_{\langle 1,1,0,0\rangle} E_{\langle 1,1,0,0\rangle}^{*}+e_{\langle 0,1,1,0\rangle} E_{\langle 0,1,1,0\rangle}^{*}+e_{\langle 0,1,0,1\rangle} E_{\langle 0,1,0,1\rangle}^{*} \\
& +e_{\langle 1,1,1,0\rangle} E_{\langle 1,1,1,0\rangle}^{*}+e_{\langle 1,1,0,1\rangle} E_{\langle 1,1,0,1\rangle}^{*}+e_{\langle 0,1,1,1\rangle} E_{\langle 0,1,1,1\rangle}^{*} \\
& +e_{\langle 1,1,1,1\rangle} E_{\langle 1,1,1,1\rangle}^{*}+e_{\langle 1,2,1,1\rangle} E_{\langle 1,2,1,1\rangle}^{*}+\cdots
\end{aligned}
$$

where the dots stand for the negatively charged terms, eg $e_{\langle-1,0,0,0\rangle} E_{\langle-1,0,0,0\rangle}^{*}$. The projector acts on operators:

$$
\begin{aligned}
P\left(N_{D_{4}}\right)= & \sum_{1 \leq i \leq 4} P\left(h_{i}\right) H_{i}^{*}+\sum_{\alpha \in \Phi^{+}}\left(P\left(e_{\alpha}\right) E_{\alpha}^{*}+P\left(e_{-\alpha}\right) E_{-\alpha}^{*}\right) \\
= & P\left(h_{1}\right) H_{1}^{*}+P\left(h_{2}\right) H_{2}^{*}+P\left(h_{3}\right) H_{3}^{*}+P\left(h_{4}\right) H_{4}^{*} \\
& +P\left(e_{\langle \pm 1,0,0,0\rangle}\right) E_{\langle \pm \pm 1,0,0,0\rangle}^{*}+P\left(e_{\langle 0, \pm 1,0,0\rangle}\right) E_{\langle 0, \pm 1,0,0\rangle}^{*} \\
& +P\left(e_{\langle 0,0, \pm 1,0\rangle}\right) E_{\langle 0,0, \pm 1,0\rangle}^{*}+P\left(e_{\langle 0,0,0, \pm 1\rangle}\right) E_{\langle 0,0,0, \pm 1\rangle}^{*} \\
& +P\left(e_{\langle \pm 1, \pm 1,0,0\rangle}\right) E_{\langle \pm 1, \pm 1,0,0\rangle}^{*}+P\left(e_{\langle 0, \pm 1, \pm 1,0\rangle}\right) E_{\langle 0, \pm 1, \pm 1,0\rangle}^{*} \\
& +P\left(e_{\langle 0, \pm 1,0, \pm 1\rangle}\right) E_{\langle 0, \pm 1,0, \pm 1\rangle}^{*}+P\left(e_{\langle \pm 1, \pm 1, \pm 1,0\rangle}\right) E_{\langle \pm 1, \pm 1, \pm 1,0\rangle}^{*} \\
& +P\left(e_{\langle \pm 1, \pm 1,0, \pm 1\rangle}\right) E_{\langle \pm 1, \pm 1,0, \pm 1\rangle}^{*}+P\left(e_{\langle 0, \pm 1, \pm 1, \pm 1\rangle}\right) E_{\langle 0, \pm 1, \pm 1, \pm 1\rangle}^{*} \\
& +P\left(e_{\langle \pm 1, \pm 1, \pm 1, \pm 1\rangle}\right) E_{\langle \pm 1, \pm 1, \pm 1, \pm 1\rangle}^{*}+P\left(e_{\langle \pm 1, \pm 2, \pm 1, \pm 1\rangle}\right) E_{\langle \pm 1, \pm 2, \pm 1, \pm 1\rangle}^{*} \\
= & h_{1} H_{1}^{*}+h_{2} H_{2}^{*}+\frac{h_{3}+h_{4}}{2} H_{3}^{*}+\frac{\left(h_{3}+h_{4}\right)}{2} H_{4}^{*} \\
& +e_{\langle \pm 1,0,0,0\rangle} E_{\langle \pm 1,0,0,0\rangle}^{*}+e_{\langle 0, \pm 1,0,0\rangle} E_{\langle 0, \pm 1,0,0\rangle}^{*}
\end{aligned}
$$




$$
\begin{aligned}
& +\frac{e_{\langle 0,0, \pm 1,0\rangle}+e_{\langle 0,0,0, \pm 1\rangle}}{2} E_{\langle 0,0, \pm 1,0\rangle}^{*}+\frac{e_{\langle 0,0, \pm 1,0\rangle}+e_{\langle 0,0,0, \pm 1\rangle}}{2} E_{\langle 0,0,0, \pm 1\rangle}^{*} \\
& +e_{\langle \pm 1, \pm 1,0,0\rangle} E_{\langle \pm 1, \pm 1,0,0\rangle}^{*}+\frac{e_{\langle 0, \pm 1, \pm 1,0\rangle}+e_{\langle 0, \pm 1,0, \pm 1\rangle}}{2} E_{\langle 0, \pm 1, \pm 1,0\rangle}^{*} \\
& +\frac{e_{\langle 0, \pm 1, \pm 1,0\rangle}+e_{\langle 0, \pm 1,0, \pm 1\rangle}}{2} E_{\langle 0, \pm 1,0, \pm 1\rangle}^{*}+\frac{e_{\langle \pm 1, \pm 1, \pm 1,0\rangle}+e_{\langle \pm 1, \pm 1,0, \pm 1\rangle}}{2} E_{\langle \pm 1, \pm 1, \pm 1,0\rangle}^{*} \\
& +\frac{e_{\langle \pm 1, \pm 1, \pm 1,0\rangle}+e_{\langle \pm 1, \pm 1,0, \pm 1\rangle}}{2} E_{\langle \pm 1, \pm 1,0, \pm 1\rangle}^{*}+e_{\langle 0, \pm 1, \pm 1, \pm 1\rangle} E_{\langle 0, \pm 1, \pm 1, \pm 1\rangle}^{*} \\
& +e_{\langle \pm 1, \pm 1, \pm 1, \pm 1\rangle} E_{\langle \pm 1, \pm 1, \pm 1, \pm 1\rangle}^{*}+e_{\langle \pm 1, \pm 2, \pm 1, \pm 1\rangle} E_{\langle \pm 1, \pm 2, \pm 1, \pm 1\rangle}^{*} \\
& =h_{ \pm 1} H_{ \pm 1}^{*}+h_{2} H_{2}^{*}+\left(h_{3}+h_{4}\right) \frac{H_{3}^{*}+H_{4}^{*}}{2} \\
& +e_{\langle \pm 1,0,0,0\rangle} E_{\langle \pm 1,0,0,0\rangle}^{*}+e_{\langle 0, \pm 1,0,0\rangle} E_{\langle 0, \pm 1,0,0\rangle}^{*} \\
& +\left(e_{\langle 0,0, \pm 1,0\rangle}+e_{\langle 0,0,0, \pm 1\rangle}\right) \frac{E_{\langle 0,0, \pm 1,0\rangle}^{*}+E_{\langle 0,0,0, \pm 1\rangle}^{*}}{2}+e_{\langle \pm 1, \pm 1,0,0\rangle} E_{\langle \pm 1, \pm 1,0,0\rangle}^{*} \\
& +\left(e_{\langle 0, \pm 1, \pm 1,0\rangle}+e_{\langle 0, \pm 1,0, \pm 1\rangle}\right) \frac{E_{\langle 0, \pm 1, \pm 1,0\rangle}^{*}+E_{\langle 0, \pm 1,0, \pm 1\rangle}^{*}}{2} \\
& +\left(e_{\langle \pm 1, \pm 1, \pm 1,0\rangle}+e_{\langle \pm 1, \pm 1,0, \pm 1\rangle}\right) \frac{E_{\langle \pm 1, \pm 1, \pm 1,0\rangle}^{*}+E_{\langle \pm 1, \pm 1,0, \pm 1\rangle}^{*}}{2} \\
& +e_{\langle 0, \pm 1, \pm 1, \pm 1\rangle} E_{\langle 0, \pm 1, \pm 1, \pm 1\rangle}^{*} \\
& +e_{\langle \pm 1, \pm 1, \pm 1, \pm 1\rangle} E_{\langle \pm 1, \pm 1, \pm 1, \pm 1\rangle}^{*}+e_{\langle \pm 1, \pm 2, \pm 1, \pm 1\rangle} E_{\langle \pm 1, \pm 2, \pm 1, \pm 1\rangle}^{*} \\
& =\tilde{h}_{1} \tilde{H}_{1}^{*}+\tilde{h}_{2} \tilde{H}_{2}^{*}+\tilde{h}_{3} \tilde{H}_{3}^{*} \\
& +\tilde{e}_{\langle \pm 1,0,0\rangle} \tilde{E}_{\langle \pm 1,0,0\rangle}^{*}+\tilde{e}_{\langle 0, \pm 1,0\rangle} \tilde{E}_{\langle 0, \pm 1,0\rangle}^{*}+\tilde{e}_{\langle 0,0, \pm 1\rangle} \tilde{E}_{\langle 0,0, \pm 1\rangle}^{*} \\
& +\tilde{e}_{\langle \pm 1, \pm 1,0\rangle} \tilde{E}_{\langle \pm 1, \pm 1,0\rangle}^{*}+\tilde{e}_{\langle 0, \pm 1, \pm 1\rangle} \tilde{E}_{\langle 0, \pm 1, \pm 1\rangle}^{*} \\
& +\tilde{e}_{\langle \pm 1, \pm 1, \pm 1\rangle} \tilde{E}_{\langle \pm 1, \pm 1, \pm 1\rangle}^{*}+\tilde{e}_{\langle 0, \pm 1, \pm 2\rangle} \tilde{E}_{\langle 0, \pm 1, \pm 2\rangle}^{*} \\
& +\tilde{e}_{\langle \pm 1, \pm 1, \pm 2\rangle} \tilde{E}_{\langle \pm 1, \pm 1, \pm 2\rangle}^{*}+\tilde{e}_{\langle \pm 1, \pm 2, \pm 2\rangle} \tilde{E}_{\langle \pm 1, \pm 2, \pm 2\rangle}^{*} \\
& =N_{B_{3}}
\end{aligned}
$$

where we defined

$$
\begin{aligned}
\tilde{h}_{3} & =h_{3}+h_{4}=2\left(\varphi_{3}+\varphi_{4}\right)-2\left(\varphi_{2,1}+\varphi_{2,2}\right) \\
\tilde{e}_{\langle 0,0,1\rangle} & =e_{\langle 0,0,1,0\rangle}+e_{\langle 0,0,0,1\rangle}=u_{3}^{+}+u_{4}^{+} \\
\tilde{e}_{\langle 0,1,2\rangle} & =e_{\langle 0,1,1,1\rangle}=\sum_{a=1,2} \frac{u_{2, a}^{+} u_{3}^{+} u_{4}^{+}}{\left(\varphi_{2, a}-\varphi_{3}\right)\left(\varphi_{2, a}-\varphi_{4}\right)}
\end{aligned}
$$

and the remaining operators follow the same pattern $\tilde{e}_{\langle a, b, 2 c\rangle}=e_{\langle a, b, c, c\rangle}$ or $\tilde{e}_{\langle a, b, c\rangle}=$ $e_{\langle a, b, c, 0\rangle}+e_{\langle a, b, 0, c\rangle}$ if $c \neq 0$ and $\tilde{e}_{\langle a, b, 0\rangle}=e_{\langle a, b, 0,0\rangle}$ otherwise. Notice a feature common to components of the moment map on which $P$ acts non-trivially: the prefactor from the operator 
becomes the inverse multiplicity required in the definition of the new dual basis, e.g.

$$
\begin{aligned}
P\left(h_{3}\right) H_{3}^{*}+P\left(h_{4}\right) H_{4}^{*} & =\frac{h_{3}+h_{4}}{\#_{3}} H_{3}^{*}+\frac{h_{3}+h_{4}}{\#_{3}} H_{4}^{*} \\
& =\left(h_{3}+h_{4}\right) \frac{H_{3}^{*}+H_{4}^{*}}{\#_{3}} \\
& =\tilde{h}_{3} \frac{\#_{3} \tilde{H}_{3}^{*}}{\#_{3}} \\
& =\tilde{h}_{3} \tilde{H}_{3}^{*} .
\end{aligned}
$$

Just as $N_{D_{4}}$ satisfies certain matrix relations which identify the space it parametrises as the (closure of the) minimal nilpotent orbit of $D_{4}$, so does $N_{B_{3}}$ obey several relations appropriate for a $B_{3}$ nilpotent orbit. The space should be an orbifold of the minimal orbit of $D_{4}$, so it should in particular have the same quaternionic dimension, namely 5 . That is precisely the dimension of the next-to-minimal orbit of $B_{3}$ with the HWG [30]

$$
\operatorname{HWG}\left(t, \mu_{i}\right)=\frac{1}{\left(1-\mu_{2} t^{2}\right)\left(1-\mu_{1}^{2} t^{4}\right)}
$$

This space is parametrised by a matrix $M$ satisfying the relations (computed using standard plethystic techniques) ${ }^{13}$

$$
\begin{aligned}
t^{4}[000]: & \operatorname{tr} N^{2}=0 \\
t^{4}[002]: & N \wedge N=0 \\
t^{6}[010]: & N^{3}=0
\end{aligned}
$$

We describe a relation by its $R$-symmetry weight appearing in the exponent of $t$ and the global symmetry representation in which it transforms. This often, but not always, specifies the tensorial form of the relation, which we provide on the other side of the colon. The notation $N \wedge N$ should be understood as the contraction $\sum_{l m n o} \epsilon_{i j k l m n o} N_{l m} N_{n o}$ with the rank 7 antisymmetric invariant tensor of $\mathfrak{s o}(7)$.

One can check that the moment map $N_{B_{3}}$ satisfies the identities in (3.24) modulo abelianised relations. To show that there exist no other independent relations, or generators for that matter, one can calculate the Hilbert series of the ring as described below. This computation shows that indeed (3.24) form the complete set of relations for the next-tominimal orbit of $\mathfrak{s o}(7)$. Note that this is an instance of Case 2 of the Kostant-Brylinski table 2 .

\subsection{Wreathed quivers}

The previous section establishes that some Coulomb branches can be orbifolded by a quiver automorphism. We will now argue that the orbifold can also be recovered as the Coulomb branch of the original quiver after gauging its automorphism. It is natural to ask if the

\footnotetext{
${ }^{13} \mathrm{~A}$ similar set of relations appears in [31], albeit for the next-to-minimal orbit of $D_{4}$. The methods employed therein can be extended to the present case: given a general nilpotent orbit, one can construct the quiver for which it is the Higgs branch and look for matrix relations implied by the $F$-terms.
} 
resulting theory is also a quiver theory which could be studied without reference to the original, ungauged theory. This is indeed possible, albeit at the cost of generalising the notion of a quiver theory to wreathed quivers.

Traditionally a quiver theory is described by a quiver diagram in which nodes represent gauge or flavor groups and links represent appropriately charged matter. Wreathed quiver theories add wreathed legs denoted by $(\cdot)$ \ $S_{n}$ with an associated wreathing group $S_{n}$. See figure 3 for two prototypical examples. The top right quiver has a single wreathed node while the bottom right quiver is an example of a quiver with a longer wreathed leg.

Abelianisation of wreathed quivers. The Coulomb branch of a wreathed quiver can be studied through abelianisation with relatively minor changes, but it is cumbersome to write them down in full generality. We find much greater clarity in (entirely equivalent) abelianised calculations performed on discretely gauged non-wreathed quivers. In practice, this amounts to keeping the indices, Poisson and abelianised chiral structure from the non-wreathed quiver while imposing invariance under the projector 3.12. For illustrative purposes, and to draw a link to [5, 6], we present two particularly simple examples depicted in figure 3.

There are very few new elements in the wreathed quiver theory depicted in the top right quiver of figure 3 . The third node brings six variables $u_{3, a}^{ \pm}$and $\varphi_{3, a}, a \in\{1,2\}$, much like a $\mathrm{U}(2)$ node would. The wreathing group acts similarly to a Weyl group in that it permutes the index $a$ and all physical operators are invariant under it.

Abelianised relations on the middle node read

$$
u_{2, a}^{+} u_{2, a}^{-}=-\frac{\left(\varphi_{2, a}-M_{2}\right)\left(\varphi_{2, a}-\varphi_{1}\right)\left(\varphi_{2, a}-\varphi_{3,1}\right)\left(\varphi_{2, a}-\varphi_{3,2}\right)}{\left(\varphi_{2,1}-\varphi_{2,2}\right)^{2}}
$$

and the relations on the third node are essentially unchanged:

$$
u_{3, a}^{+} u_{3, a}^{-}=-\left(\varphi_{3, a}-\varphi_{2,1}\right)\left(\varphi_{3, a}-\varphi_{2,2}\right) .
$$

Interestingly, the latter can be read in two ways: either as the relation of a U(1) ₹ $S_{2}$ node, or as

$$
u_{3, a}^{+} u_{3, a}^{-}=-\frac{\left(\varphi_{3, a}-\varphi_{2,1}\right)\left(\varphi_{3, a}-\varphi_{2,2}\right)}{\left(\varphi_{3,1}-\varphi_{3,2}\right)^{2}}\left(\varphi_{3,1}-\varphi_{3,2}\right)^{2},
$$

which is appropriate for a $\mathrm{U}(2)$ node with adjoint matter. This explains why in $[5,6]$ a "bouquet" of $n \mathrm{U}(1)$ nodes combined into $\mathrm{U}(n)$ with adjoint matter: at the level of the Coulomb branch, there is no difference between $\mathrm{U}(1)$ \& $S_{n}$ and $\mathrm{U}(n)$ with adjoint matter.

The case of the bottom right quiver in figure 3 is slightly more subtle. The first and second gauge nodes, which are inside the scope of a two-fold wreathing, each come with six variables $u_{3, a}^{ \pm}$and $\varphi_{3, a}, a \in\{1,2\}$. However, the pattern of abelianised relations, which can be determined by consistency with the discrete gauging of the bottom left quiver in figure 3 , is as follows:

$$
\begin{aligned}
u_{1, a}^{+} u_{1, a}^{-} & =-\left(\varphi_{1, a}-M_{1}\right)\left(\varphi_{1, a}-\varphi_{2, a}\right) \\
u_{2, a}^{+} u_{2, a}^{-} & =-\left(\varphi_{2, a}-\varphi_{1, a}\right)\left(\varphi_{2, a}-\varphi_{3}\right) \\
u_{3}^{+} u_{3}^{-} & =-\left(\varphi_{3}-\varphi_{2,1}\right)\left(\varphi_{3}-\varphi_{2,2}\right)
\end{aligned}
$$


Note in particular that the index $a$ "stretches" across several nodes (but not the mass variable, which is shared by all legs). The wreathing group $S_{2}$ again acts on this index, and invariance under it is a necessary prerequisite for operator physicality. The Coulomb branch has $C_{3}$ symmetry and the moment map parametrises the next-to-minimal nilpotent orbit of this algebra. Its components include:

$$
\begin{aligned}
e_{\langle \pm 1,0,0\rangle}= & u_{1,1}^{ \pm}+u_{1,2}^{ \pm} \\
e_{\langle 0, \pm 1,0\rangle}= & u_{2,1}^{ \pm}+u_{2,2}^{ \pm} \\
e_{\langle 0,0, \pm 1\rangle}= & u_{3}^{ \pm} \\
e_{\langle \pm 1, \pm 1,0\rangle}= & \frac{u_{1,1}^{ \pm} u_{2,1}^{ \pm}}{\varphi_{1,1}-\varphi_{2,1}}+\frac{u_{1,2}^{ \pm} u_{2,2}^{ \pm}}{\varphi_{1,2}-\varphi_{2,2}} \\
e_{\langle 0, \pm 1, \pm 1\rangle}= & \frac{u_{2,1}^{ \pm} u_{3}^{ \pm}}{\varphi_{2,1}-\varphi_{3}}+\frac{u_{2,2}^{ \pm} u_{3}^{ \pm}}{\varphi_{2,2}-\varphi_{3}} \\
e_{\langle \pm 1, \pm 1, \pm 1\rangle}= & \frac{u_{1,1}^{ \pm} u_{2,1}^{ \pm} u_{3,1}^{ \pm}}{\left(\varphi_{1,1}-\varphi_{2,1}\right)\left(\varphi_{2,1}-\varphi_{3}\right)} \\
& +\frac{u_{1,2}^{ \pm} u_{2,2}^{ \pm} u_{3,1}^{ \pm}}{\left(\varphi_{1,2}-\varphi_{2,2}\right)\left(\varphi_{2,2}-\varphi_{3}\right)} \\
e_{\langle 0, \pm 2, \pm 1\rangle}= & \frac{u_{2,1}^{ \pm} u_{2,2}^{ \pm} u_{3}^{ \pm}}{\left(\varphi_{2,1}-\varphi_{3}\right)\left(\varphi_{2,2}-\varphi_{3}\right)} \\
e_{\langle \pm 1, \pm 2, \pm 1\rangle}= & \frac{u_{1,1}^{ \pm} u_{2,1}^{ \pm} u_{2,2}^{ \pm} u_{3,1}^{ \pm}}{\left(\varphi_{1,1}-\varphi_{2,1}\right)\left(\varphi_{2,1}-\varphi_{3}\right)\left(\varphi_{2,2}-\varphi_{3}\right)} \\
& +\frac{u_{1,2}^{ \pm} u_{2,1}^{ \pm} u_{2,2}^{ \pm} u_{3,1}^{ \pm}}{\left(\varphi_{1,2}-\varphi_{2,1}\right)\left(\varphi_{2,1}-\varphi_{3}\right)\left(\varphi_{2,2}-\varphi_{3}\right)} \\
e_{\langle \pm 2, \pm 2, \pm 1\rangle}= & \frac{u_{1,1}^{ \pm} u_{1,2}^{ \pm} u_{2,1}^{ \pm} u_{2,2}^{ \pm} u_{3,1}^{ \pm}}{\left(\varphi_{1,1}-\varphi_{2,1}\right)\left(\varphi_{1,2}-\varphi_{2,2}\right)\left(\varphi_{2,1}-\varphi_{3}\right)\left(\varphi_{2,2}-\varphi_{3}\right)} \\
h_{1}= & 2\left(\varphi_{1,1}+\varphi_{1,2}\right)-\left(\varphi_{2,1}+\varphi_{2,2}\right) \\
h_{2}= & -\left(\varphi_{1,1}+\varphi_{3,1}\right)+2\left(\varphi_{2,1}+\varphi_{2,2}\right)-2 \varphi_{3} \\
h_{3}= & -\left(\varphi_{2,1}+\varphi_{2,2}\right)+2 \varphi_{3} \\
&
\end{aligned}
$$

\subsection{Monopole formula for wreathed quivers}

Consider now a wreathed quiver. To compute the monopole formula, we need to replace the Weyl group in (2.25) with an appropriate discrete group, necessarily a subgroup of $S_{r}$ which contains the Weyl group $W$ of the gauge group $G$ and leaves $\Delta(m)$ invariant. Generically, several such discrete groups exist. Our choice, which we dub $W_{\Gamma}$, is the wreath product of the Weyl group $W$ by the wreathing group $\Gamma$ :

$$
W_{\Gamma}=W \imath \Gamma, \quad W \subseteq W_{\Gamma} \subseteq S_{r} .
$$

Formula (2.25) generalises readily for such a group, setting

$$
\operatorname{HS}_{\Gamma}(t)=\frac{1}{\left|W_{\Gamma}\right|} \sum_{m \in \mathbb{Z}^{r}} \sum_{\gamma \in W_{\Gamma}(m)} \frac{t^{2 \Delta(m)}}{\operatorname{det}\left(1-t^{2} \gamma\right)} .
$$

This is the monopole formula for the wreathed quiver. 
A comment on computational complexity. The monopole formula in the form (3.44) is very time-consuming to evaluate numerically in a series expansion in $t$. For such a task, it is preferable to preprocess it somewhat, using the high level of symmetry that it presents. In particular, if the group $\Gamma$ can be written as a product of two groups $W_{\Gamma}=W_{1} \times W_{2}$, then one can split the summation into two sums.

This procedure involves finding a subset of $\mathbb{Z}^{r}$ which contains exactly one element of each orbit of $W_{\Gamma}$. In the context of Weyl groups, or more generally Coxeter groups, this is called a fundamental chamber. For instance, if $W_{\Gamma}=W$ as in (2.22), then this group can be used to order the magnetic charges in increasing order for each node. Then one uses the identity

$$
P_{\mathrm{U}(n)}\left(t^{2} ; m_{1}, \ldots, m_{N}\right):=P_{S_{n}}\left(t ; m_{1}, \ldots, m_{n}\right)=\frac{1}{n !} \sum_{\gamma \in S_{n}(m)} \frac{1}{\operatorname{det}\left(1-t^{2} \gamma\right)}
$$

for the Casimir factors as defined in the appendix of [2]. This is done in the usual way of presenting the monopole formula.

For wreathed quivers, $W_{\Gamma}$ does not decompose in general as a direct product of symmetry groups. One can introduce symmetry factors exactly as in (3.45), via

$$
P_{W_{\Gamma}}\left(t^{2} ; m\right)=\frac{1}{\left|W_{\Gamma}\right|} \sum_{\gamma \in W_{\Gamma}(m)} \frac{1}{\operatorname{det}\left(1-t^{2} \gamma\right)} .
$$

The formula (3.44) can then be rewritten

$$
\operatorname{HS}_{\Gamma}(t)=\sum_{m \in \operatorname{Weyl}(G(\Gamma)} P_{W_{\Gamma}}(t ; m) t^{2 \Delta(m)},
$$

where $\operatorname{Weyl}(G \succ \Gamma)$ is a principal Weyl chamber for the group $G \imath \Gamma$. We now illustrate the procedure on three examples and most explicitly on the third.

Example 1: subgroups of $\boldsymbol{S}_{\mathbf{3}}$. Consider the quiver corresponding to the affine $D_{4}$ Dynkin diagram (see the first column of table 11). One of the four rank-one nodes is a flavor node, so we can define the graph by the vertices

$$
V=\{a, b, c, d\} \quad F=\{e\}
$$

where $a$ denotes the central node, and

$$
E=\{(a, b),(a, c),(a, d)\} \quad E^{\prime}=\{(a, e)\} .
$$

The corresponding ranks are $n_{a}=2, n_{b}=n_{c}=n_{d}=n_{e}=1$. The total gauge group is $G=\mathrm{U}(2) \times \mathrm{U}(1)^{3}$ with rank $r=5$. The Weyl group is $W=S_{2}$. The magnetic charges are elements $m=\left(m_{a, 1}, m_{a, 2}, m_{b}, m_{c}, m_{d}\right) \in \mathbb{Z}^{5}$ and the conformal dimension is given by

$$
2 \Delta(m)=\sum_{i=1}^{2}\left(\left|m_{a, i}-m_{b}\right|+\left|m_{a, i}-m_{c}\right|+\left|m_{a, i}-m_{d}\right|+\left|m_{a, i}\right|\right)-2\left|m_{a, 1}-m_{a, 2}\right| .
$$




\begin{tabular}{|c|c|c|}
\hline Name & Generators & Cardinality \\
\hline Trivial & - & 1 \\
$S_{2}$ & $(12)$ & 2 \\
Double transposition & $(12)(34)$ & 2 \\
$\mathbb{Z}_{4}$ & $(1234)$ & 4 \\
Normal Klein & $(12)(34),(13)(24)$ & 4 \\
Non-normal Klein & $(12),(34)$ & 4 \\
Dih $_{4}$ & $(1234),(13)$ & 8 \\
$\mathbb{Z}_{3}$ & $(123)$, & 3 \\
$S_{3}$ & $(12),(13)$ & 6 \\
$A_{4}$ & $(123),(124)$ & 12 \\
$S_{4}$ & $(12),(13),(14)$ & 24 \\
\hline
\end{tabular}

Table 4. Subgroups of $S_{4}$.

The group $S_{5}$ includes 156 subgroups which can be gathered into 19 conjugacy classes. These 19 classes are partially ordered and form a Hasse diagram. For $\Delta$ to be invariant, we have to select those groups $\Gamma$ which are subgroups of $S_{2} \times S_{3}$ (this is also known as the dihedral group $D_{12}$ ), and moreover to satisfy (3.43) the groups $\Gamma$ have to contain $S_{2}$ as a subgroup. Out of the 19 classes of subgroups, 9 are subgroups of $D_{12}$, and out of these 9,6 contain a $S_{2}$ as a subgroup. However there are two equivalent but non-conjugate $S_{2}$ subgroups of $D_{12}$, and we have to pick one of them. We are then left with 4 classes of subgroups, which can be identified with the 4 classes of subgroups of $S_{3}\left(S_{3}, \mathbb{Z}_{3}, \mathbb{Z}_{2}\right.$ and 1$)$. Clearly, in this simple example this analysis is slightly superfluous and the result could have been guessed. We end up with 4 inequivalent groups $\Gamma$, and we can readily evaluate the expression (3.44):

$$
\begin{aligned}
\mathrm{HS}_{\mathbb{Z}_{2}} & =\frac{\left(1+t^{2}\right)\left(1+17 t^{2}+48 t^{4}+17 t^{6}+t^{8}\right)}{\left(1-t^{2}\right)^{10}} \\
\mathrm{HS}_{\mathbb{Z}_{2} \times \mathbb{Z}_{2}} & =\frac{\left(1+t^{2}\right)\left(1+10 t^{2}+20 t^{4}+10 t^{6}+t^{8}\right)}{\left(1-t^{2}\right)^{10}} \\
\mathrm{HS}_{\mathbb{Z}_{2} \times \mathbb{Z}_{3}} & =\frac{\left(1+t^{2}\right)\left(1+3 t^{2}+20 t^{4}+3 t^{6}+t^{8}\right)}{\left(1-t^{2}\right)^{10}} \\
\mathrm{HS}_{\mathbb{Z}_{2} \times S_{3}} & =\frac{\left(1+t^{2}\right)\left(1+3 t^{2}+6 t^{4}+3 t^{6}+t^{8}\right)}{\left(1-t^{2}\right)^{10}}
\end{aligned}
$$

which identify the spaces as the (closure of the) minimal nilpotent orbit of $\mathrm{SO}(8)$, next to minimal of $\mathrm{SO}(7)$, double cover of the subregular orbit of $G_{2}$ [14], and the subregular orbit of $G_{2}$. 


\begin{tabular}{|c|c|c|}
\hline Subgroup & Perturbative Hilbert series & PLog \\
\hline Trivial & $1+28 t^{2}+300 t^{4}+1925 t^{6}+8918 t^{8}+\ldots$ & $28 t^{2}-106 t^{4}+833 t^{6}-8400 t^{8}+\ldots$ \\
$S_{2}$ & $1+21 t^{2}+195 t^{4}+1155 t^{6}+5096 t^{8}+\ldots$ & $21 t^{2}-36 t^{4}+140 t^{6}-784 t^{8}+\ldots$ \\
Double transposition & $1+16 t^{2}+160 t^{4}+985 t^{6}+4522 t^{8}+\ldots$ & $16 t^{2}+24 t^{4}-215 t^{6}+522 t^{8}+\ldots$ \\
$\mathbb{Z}_{4}$ & $1+9 t^{2}+83 t^{4}+497 t^{6}+2270 t^{8}+\ldots$ & $9 t^{2}+38 t^{4}-10 t^{6}-586 t^{8}+\ldots$ \\
Normal Klein & $1+10 t^{2}+90 t^{4}+515 t^{6}+2324 t^{8}+\ldots$ & $10 t^{2}+35 t^{4}-55 t^{6}-396 t^{8}+\ldots$ \\
Non-normal Klein & $1+15 t^{2}+125 t^{4}+685 t^{6}+2898 t^{8}+\ldots$ & $15 t^{2}+5 t^{4}-70 t^{6}+273 t^{8}+\ldots$ \\
Dih $_{4}$ & $1+9 t^{2}+69 t^{4}+356 t^{6}+1485 t^{8}+\ldots$ & $9 t^{2}+24 t^{4}-25 t^{6}-165 t^{8}+\ldots$ \\
$\mathbb{Z}_{3}$ & $1+14 t^{2}+118 t^{4}+693 t^{6}+3094 t^{8}+\ldots$ & $14 t^{2}+13 t^{4}-49 t^{6}-56 t^{8}+\ldots$ \\
$S_{3}$ & $1+14 t^{2}+104 t^{4}+539 t^{6}+2184 t^{8}+\ldots$ & $14 t^{2}-t^{4}-7 t^{6}+7 t^{8}+\ldots$ \\
$A_{4}$ & $1+8 t^{2}+48 t^{4}+223 t^{6}+896 t^{8}+\ldots$ & $8 t^{2}+12 t^{4}+7 t^{6}+0 t^{8}+\ldots$ \\
$S_{4}$ & $1+8 t^{2}+48 t^{4}+210 t^{6}+771 t^{8}+\ldots$ & $8 t^{2}+12 t^{4}-6 t^{6}-21 t^{8}+\ldots$ \\
\hline
\end{tabular}

Table 5. Wreathed quivers obtained from the affine $D_{4}$ quiver by acting on the legs by all subgroups of $S_{4}$.

Example 2: subgroups of $\boldsymbol{S}_{\mathbf{4}}$. We now consider the same quiver as in the previous example, namely the affine $D_{4}$ quiver, but we use the fact that the gauge group of the theory is really

$$
\frac{\mathrm{U}(1)^{4} \times \mathrm{U}(2)}{\mathrm{U}(1)}
$$

where the U(1) acts diagonally. This form makes the $S_{4}$ symmetry of the quiver explicit, and this $S_{4}$ contains as a subgroup the $S_{3}$ which is studied in the previous example. Following the approach of this section, one can define a wreathed quiver for each conjugacy class of subgroups of $S_{4}$. Part of the results presented here already appear in unpublished summer work by Siyul Lee [32], where the cycle index technique was used. The group $S_{4}$ admits 30 subgroups that can be organized into 11 conjugacy classes, as listed in table 4 , where we give a name to each class of subgroups.

For each subgroup, one can compute the Hilbert series (3.44). The results are gathered in figure 4, where they are arranged in the shape of the Hasse diagram of the subgroups of $S_{4}$. We give some details about the computation in appendix C. We also give the first orders of the series expansions of these Hilbert series, along with their plethystic logarithms, in table 5 . The coefficient of the $t^{2}$ term gives the dimension of the isometry group of the Coulomb branch. 


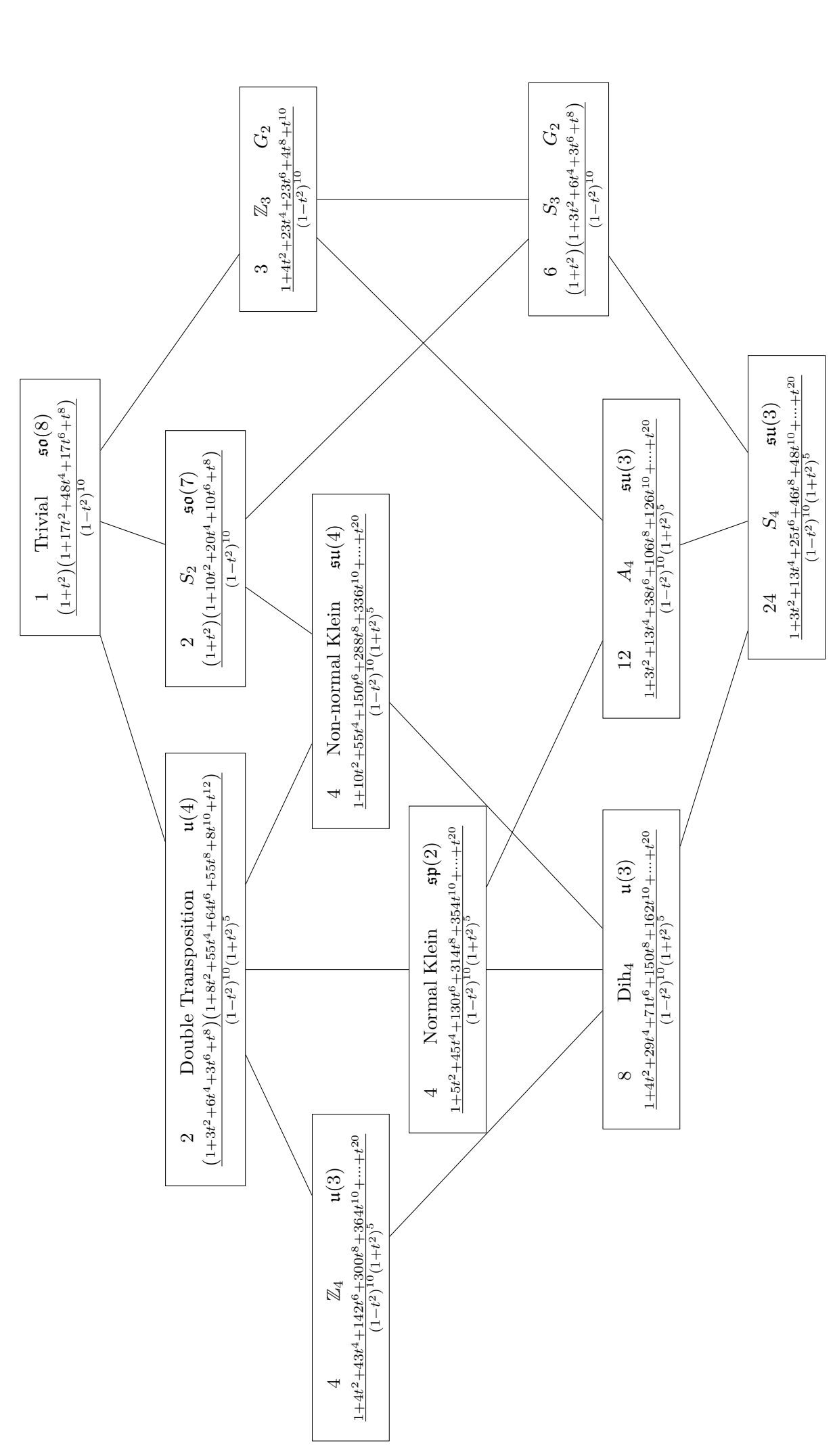

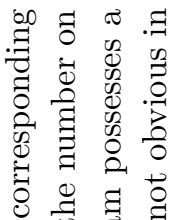

政

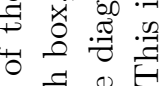

矛通过

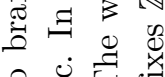

염 节色

응 迎

ช

年

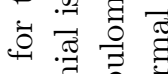

马

要

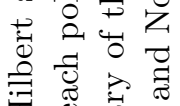

䟚

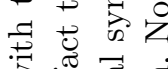

施

जो

岁点量

:.0

कै

ㄴㅇㅛ

设

沿

은

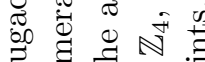

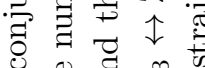

ㅎำ

g.

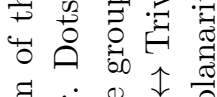

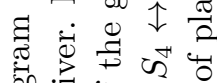

要表宁

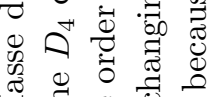

毒节离

+i

记

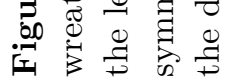

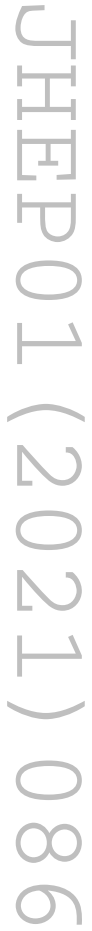


Example 3: wreath product of non Abelian groups. We now consider the quiver

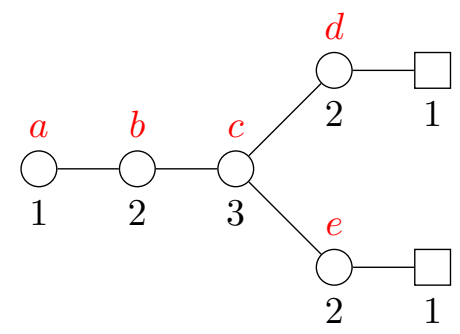

whose Coulomb branch is the closure of the nilpotent orbit of $\mathfrak{s o}(10)$ associated with the partition $\left[2^{4}, 1^{2}\right]$. The letters in red give our assignment of magnetic charge for the various gauge groups. The rank is $r=10$ and the Weyl group is $W=S_{1} \times S_{2} \times S_{3} \times S_{2} \times S_{2}$. In order to preserve $\Delta$, we need a symmetry of the quiver, which is given by permutation of the two legs containing the nodes $d$ and $e$. So there are only two allowed groups $W_{\Gamma}$, namely $W_{\Gamma}=W$ and an extension $W_{\Gamma}$ of $W$ of index 2. Let's focus on this second group.

The factors $S_{1} \times S_{2} \times S_{3}$ in $W$ are unaffected by the permutation, so we omit them in the matrix discussion that follows. The commutant of this part in $S_{10}$ is $S_{4}$, which acts by permuting the four magnetic fugacities $\left(d_{1}, d_{2}, e_{1}, e_{2}\right)$. The group $W_{\Gamma}$ is then the product $W_{\Gamma}=S_{1} \times S_{2} \times S_{3} \times \Gamma^{\prime}$ where $S_{2} \times S_{2} \subset \Gamma^{\prime} \subset S_{4}$. We can describe $\Gamma^{\prime}$ explicitly as generated by the following two permutation matrices:

$$
\left(\begin{array}{llll}
0 & 1 & 0 & 0 \\
1 & 0 & 0 & 0 \\
0 & 0 & 1 & 0 \\
0 & 0 & 0 & 1
\end{array}\right), \quad\left(\begin{array}{llll}
0 & 0 & 1 & 0 \\
0 & 0 & 0 & 1 \\
1 & 0 & 0 & 0 \\
0 & 1 & 0 & 0
\end{array}\right) .
$$

This group is isomorphic to the dihedral group of order 8, that we denote by $\mathrm{Dih}_{4}$. With this explicit description, it is now possible to evaluate (3.44), and one finds the Hilbert series for the Coulomb branch of the wreathed quiver,

$$
\mathrm{HS}_{S_{1} \times S_{2} \times S_{3} \times \mathrm{Dih}_{4}}=\frac{1+14 t^{2}+106 t^{4}+454 t^{6}+788 t^{8}+454 t^{10}+106 t^{12}+14 t^{14}+t^{16}}{\left(1-t^{2}\right)^{20}\left(1+t^{2}\right)^{-2}} .
$$

The corresponding HWG and other data concerning this space are gathered in the middle column of table 14 .

However, the sum involved in the computation is difficult to evaluate in practice, and it is useful to use the symmetries to avoid unnecessary repetitions, as explained above. In the present case, the sum in (3.44) for $W_{\Gamma}=S_{1} \times S_{2} \times S_{3} \times \operatorname{Dih}_{4}$ becomes (3.47) where the sum over the Weyl chamber is given by:

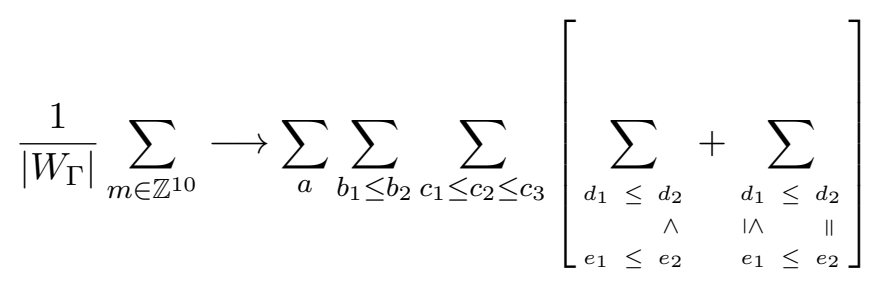


The notation here should be clear, with the charges $m=\left(a, b_{1}, b_{2}, c_{1}, c_{2}, c_{3}, d_{1}, d_{2}, e_{1}, e_{2}\right) \in$ $\mathbb{Z}^{10}$ denoted with the letters as in (3.56). The first three sums in the right hand side of (3.59) exploit in the standard way the symmetric groups, which allow to order the charges. The same principle is used to get the summation range over indices $\left(d_{1}, d_{2}, e_{1}, e_{2}\right)$. Inside the sum, one of course introduces symmetry factors (3.46). Let's now explain the summation range for the last four indices in (3.59).

$\Gamma^{\prime}$ is the dihedral group $\mathrm{Dih}_{4}$, of order 8 , or the group of symmetries of the square

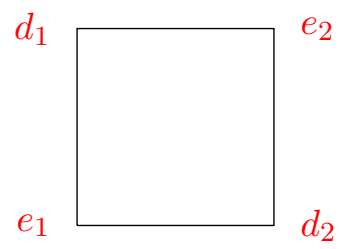

The elements of $\Gamma^{\prime}$ are listed in table 6 , with some of their properties. Without entering into the details of the theory of Coxeter groups, let us note that the Weyl chambers in $\mathbb{R}^{4}$ are delimited by subspaces fixed by the order 2 elements in the group. Formally, the Weyl group of a simple Lie algebra, the principal Weyl chamber is defined as the set of charges $m$ which satisfy the inequalities

$$
\alpha \cdot m \geq 0
$$

for every simple root $\alpha$. However, in the present case the order 2 elements don't necessarily fix a hyperplane in $\mathbb{R}^{4}$ (the -1 eigenspace can have dimension $>1$ ). The condition (3.61) then has to be replaced by a more general condition, which we now explain on our example. We leave the study of the general case, and the connection with Coxeter group theory, for future work.

The elements of order 2 in $W_{\Gamma}$ can be read from table 6 . For every element $\alpha$ of order 2 in $W_{\Gamma}$, seen as a group of endomorphisms of its representation space, we pick a basis $\left(\delta_{i}^{\alpha}\right)$ of the kernel of this endomorphism in a consistent way (with $i=1, \ldots, \operatorname{dim} \operatorname{ker}(\alpha)$ ). This is done in the third column of table 6 . The principal Weyl chamber is then defined by

$$
\delta^{\alpha} \cdot m \geq 0
$$

which generalizes (3.61). The subtlety in (3.62) comes from the cases where $\operatorname{dim} \operatorname{ker}(\alpha)>1$. When this is the case, $\delta^{\alpha} \cdot m$ is an element of $\mathbb{R}^{\operatorname{dim} \operatorname{ker}(\alpha)}$ and we need to say what we mean by $\geq$. A simple choice, which we adopt here, is to pick the lexicographic order

$$
(x, y) \leq\left(x^{\prime}, y^{\prime}\right) \Leftrightarrow y<y^{\prime} \text { or }\left(y=y^{\prime} \text { and } x \leq x^{\prime}\right) .
$$

Doing this for every order 2 element in table 6 , we get the conditions listed in the last column of that figure. Combining all these conditions together, we obtain the summation range in (3.59).

\subsection{HWG for wreathed quivers}

We now explain how to perform the orbifold at the level of the HWG. The starting point is the HWG of the Coulomb branch $\mathcal{C}$ of a quiver, which can be wreathed by a finite permutation group $\Gamma$. The goal is to compute the HWG for $\mathcal{C} / \Gamma$. 


\begin{tabular}{|c|c|c|c|}
\hline Permutation & Order & -1 eigenspace & Inequality \\
\hline Identity & 1 & & \\
\hline$d_{1} \leftrightarrow d_{2}$ & 2 & $\left(\begin{array}{llll}-1 & 1 & 0 & 0\end{array}\right)$ & $d_{1} \leq d_{2}$ \\
\hline$e_{1} \leftrightarrow e_{2}$ & 2 & $\left(\begin{array}{llll}0 & 0 & -1 & 1\end{array}\right)$ & $e_{1} \leq e_{2}$ \\
\hline$d_{1} \leftrightarrow d_{2}, e_{1} \leftrightarrow e_{2}$ & 2 & $\left(\begin{array}{cccc}-1 & 1 & 0 & 0 \\
0 & 0 & -1 & 1\end{array}\right)$ & $\begin{array}{c}e_{1}<e_{2} \text { or } \\
e_{1}=e_{2} \text { and } d_{1} \leq d_{2}\end{array}$ \\
\hline$d_{1} \leftrightarrow e_{1}, d_{2} \leftrightarrow e_{2}$ & 2 & $\left(\begin{array}{cccc}-1 & 0 & 1 & 0 \\
0 & -1 & 0 & 1\end{array}\right)$ & $\begin{array}{c}d_{2}<e_{2} \text { or } \\
d_{2}=e_{2} \text { and } d_{1} \leq e_{1}\end{array}$ \\
\hline$d_{1} \leftrightarrow e_{2}, d_{2} \leftrightarrow e_{1}$ & 2 & $\left(\begin{array}{cccc}0 & -1 & 1 & 0 \\
-1 & 0 & 0 & 1\end{array}\right)$ & $\begin{array}{c}d_{1}<e_{2} \text { or } \\
d_{1}=e_{2} \text { and } d_{2} \leq e_{1}\end{array}$ \\
\hline$d_{1} \rightarrow e_{1} \rightarrow d_{2} \rightarrow e_{2} \rightarrow d_{1}$ & 4 & & \\
\hline$d_{1} \rightarrow e_{2} \rightarrow d_{2} \rightarrow e_{1} \rightarrow d_{1}$ & 4 & & \\
\hline
\end{tabular}

Table 6. Elements of the group $\mathrm{Dih}_{4}$. In the first column, they are presented as permutations, acting on $\left(d_{1}, d_{2}, e_{1}, e_{2}\right)$. The second column is the order of the element, the third gives a basis of the -1 eigenspace in the $\left(d_{1}, d_{2}, e_{1}, e_{2}\right)$ representation. The last column displays the condition imposed by (3.62).

In the following, we give the general prescription, and at the same time we illustrate with three examples $\Gamma=\mathbb{Z}_{2}, \mathbb{Z}_{3}$ and $S_{3}$ to keep the discussion concrete. We first recall that the group $\Gamma$ has a well defined character table, which is a square matrix whose columns are labelled by conjugacy classes of elements of $\Gamma$, and whose rows are labelled by irreducible representations of $\Gamma$. For our three examples, these character tables are

\begin{tabular}{|c|cc|}
\hline$\Gamma=\mathbb{Z}_{2}$ & 1 & -1 \\
\hline Cardinality & 1 & 1 \\
\hline $\mathbf{1}$ & 1 & 1 \\
$\epsilon$ & 1 & -1 \\
\hline
\end{tabular}

\begin{tabular}{|c|ccc|}
\hline$\Gamma=\mathbb{Z}_{3}$ & 1 & $\omega$ & $\omega^{2}$ \\
\hline Cardinality & 1 & 1 & 1 \\
\hline $\mathbf{1}$ & 1 & 1 & 1 \\
$\mathbf{f}$ & 1 & $\omega$ & $\omega^{2}$ \\
$\overline{\mathbf{f}}$ & 1 & $\omega^{2}$ & $\omega$ \\
\hline
\end{tabular}

\begin{tabular}{|c|c|c|c|}
\hline$\Gamma=S_{3}$ & \multicolumn{3}{|c|}{ Id 3-cycles 2-cycles } \\
\hline Cardinality & 1 & 2 & 3 \\
\hline 1 & 1 & 1 & 1 \\
\hline$\varepsilon$ & 1 & 1 & -1 \\
\hline 2 & 2 & -1 & 0 \\
\hline
\end{tabular}

These character tables contain in each entry the trace of the matrices of the conjugacy class in the corresponding representation. One way to refine this information is to give, instead of the trace, the list (unordered, and with repetitions allowed) of the eigenvalues of these matrices. We will need these eigenvalues in equation (3.70). On our three examples, 
we get

\begin{tabular}{|c|c|c|c|c|c|c|c|c|}
\hline & & $\Gamma=\mathbb{Z}_{2}$ & \multicolumn{2}{|c|}{$\begin{array}{ll}1 & -1\end{array}$} & & \\
\hline & & & & Cardinality & 1 & & & \\
\hline & & & & 1 & $\{1\} \quad\{$ & & & \\
\hline & & & & $\epsilon$ & $\{1\}\{-$ & $-1\}$ & & \\
\hline$\Gamma=\mathbb{Z}_{3}$ & 1 & $\omega$ & $\omega^{2}$ & & $=S_{3}$ & Id & 3-cycles & 2-cycles \\
\hline Cardinality & 1 & 1 & 1 & Cardi & inality & 1 & 2 & 3 \\
\hline 1 & & $\{1\}$ & $\{1\}$ & & 1 & & & $\{1\}$ \\
\hline f & $\{1\}$ & $\{\omega\}$ & $\left\{\omega^{2}\right\}$ & & $\varepsilon$ & $\{1\}$ & $\{1\}$ & $\{-1\}$ \\
\hline$\overline{\mathbf{f}}$ & $\{1\}$ & $\left\{\omega^{2}\right\}$ & $\{\omega\}$ & & 2 & $\{1,1\}$ & $\left\{\omega, \omega^{2}\right\}$ & $\{1,-1\}$ \\
\hline
\end{tabular}

Of course in each case the sum of the eigenvalues listed in (3.66), (3.67) gives the characters (3.64), (3.65). Let us call $C_{j}$ the conjugacy classes $(j=1, \ldots, n$, with $n$ the number of conjugacy classes, and $C_{1}$ is the class of the identity element), $c_{j}=\left|C_{j}\right|$ their cardinalities, $\rho_{i}$ the irreducible representations $\left(i=1, \ldots, n\right.$, and $\rho_{1}$ is the trivial representation), and $d_{i}$ their dimensions. Finally we denote by $\Lambda_{i, j}$ the list of eigenvalues for $C_{j}$ in $\rho_{i}$. For a representation $R$ which is not irreducible, we similarly denote by $\Lambda_{R, j}$ the list of eigenvalues of the class $C_{j}$ in the representation $R$. The elements of $\Lambda_{R, j}$ are denoted $\lambda_{R, j}^{k}$ for $k=1, \ldots, \operatorname{dim} R$. This list is easily obtained from the decomposition of $R$ in irreducible representations. Note that $\lambda_{R, 1}^{k}=1$ for all $k$.

We now show how to compute the HWG for an orbifold Coulomb branch based on an initial Coulomb branch that admits a finite HWG. We say that $\operatorname{HWG}(\mathcal{C})$ is finite is there exist two lists of monomials, that we denote $\left(M_{1}, \ldots, M_{K}\right)$ and $\left(M_{1}^{\prime}, \ldots, M_{K^{\prime}}^{\prime}\right)$, in the highest weight fugacities $\left(\mu_{l}\right)$ and the variable $t$, so that the HWG is

$$
\operatorname{HWG}(\mathcal{C})=\frac{\prod_{k^{\prime}=1}^{K^{\prime}}\left(1-M_{k^{\prime}}^{\prime}\right)}{\prod_{k=1}^{K}\left(1-M_{k}\right)}
$$

We assume that $\operatorname{HWG}(\mathcal{C})$ can be written in that way; this is a non-trivial assumption, as it is known that many Coulomb branches don't satisfy it.

The fact that $\Gamma$ is a symmetry group translates into the fact that to the above expression are associated two representations $R$ and $R^{\prime}$ of $\Gamma$, not necessarily irreducible, of respective dimensions $K$ and $K^{\prime}$, such that the numerator and the denominator of the above expression transform according to these representations. Then $\operatorname{HWG}(\mathcal{C})$ can be written

$$
\operatorname{HWG}(\mathcal{C})=\frac{\prod_{k^{\prime}=1}^{K^{\prime}}\left(1-\lambda_{R^{\prime}, 1}^{k^{\prime}} M_{k^{\prime}}^{\prime}\right)}{\prod_{k=1}^{K}\left(1-\lambda_{R, 1}^{k} M_{k}\right)}
$$

From this expression it is then straightforward to write the conjectured HWG for the orbifold

$$
\operatorname{HWG}(\mathcal{C} / \Gamma)=\frac{1}{|\Gamma|} \sum_{j=1}^{n} c_{j} \times \frac{\prod_{k^{\prime}=1}^{K^{\prime}}\left(1-\lambda_{R^{\prime}, j}^{k^{\prime}} M_{k^{\prime}}^{\prime}\right)}{\prod_{k=1}^{K}\left(1-\lambda_{R, j}^{k} M_{k}\right)} .
$$


We illustrate how this formula works in practice on the example of the $D_{4}$ affine quiver. All HWGs and quivers are gathered in table 11. Consider for instance the HWGs written in terms of $G_{2}$ fugacities. The closure of the minimal nilpotent orbit of $D_{4}$ has HWG equal to $\mathrm{PE}\left[2 \mu_{1} t^{2}+\mu_{2} t^{2}+\mu_{2} t^{4}\right]$. The identification of the irreducible representations is as follows:

$$
\begin{array}{ll}
\mathbb{Z}_{2}: & \operatorname{HWG}(\mathcal{C})=\operatorname{PE}\left[\mathbf{1} \mu_{1} t^{2}+\varepsilon \mu_{1} t^{2}+\mathbf{1} \mu_{2} t^{2}+\varepsilon \mu_{2} t^{4}\right] \\
\mathbb{Z}_{3}: & \operatorname{HWG}(\mathcal{C})=\operatorname{PE}\left[\mathbf{f} \mu_{1} t^{2}+\overline{\mathbf{f}} \mu_{1} t^{2}+\mathbf{1} \mu_{2} t^{2}+\mathbf{1} \mu_{2} t^{4}\right] \\
S_{3}: & \operatorname{HWG}(\mathcal{C})=\operatorname{PE}\left[\mathbf{2} \mu_{1} t^{2}+\mathbf{1} \mu_{2} t^{2}+\varepsilon \mu_{2} t^{4}\right]
\end{array}
$$

We then use equation (3.70) to obtain

$$
\begin{aligned}
& \operatorname{HWG}\left(\mathcal{C} / \mathbb{Z}_{2}\right)=\frac{1}{2}\left(\frac{1}{\left(1-\mu_{1} t^{2}\right)^{2}\left(1-\mu_{2} t^{2}\right)\left(1-\mu_{2} t^{4}\right)}\right. \\
& \left.+\frac{1}{\left(1-\mu_{1} t^{2}\right)\left(1+\mu_{1} t^{2}\right)\left(1-\mu_{2} t^{2}\right)\left(1+\mu_{2} t^{4}\right)}\right) \\
& =\frac{1-\mu_{1}^{2} \mu_{2}^{2} t^{12}}{\left(1-\mu_{1} t^{2}\right)\left(1-\mu_{2} t^{2}\right)\left(1-\mu_{1}^{2} t^{4}\right)\left(1-\mu_{1} \mu_{2} t^{6}\right)\left(1-\mu_{2}^{2} t^{8}\right)} \text {. } \\
& \operatorname{HWG}\left(\mathcal{C} / \mathbb{Z}_{3}\right)=\frac{1}{3} \sum_{i=0}^{2} \frac{1}{\left(1-\omega^{i} \mu_{1} t^{2}\right)\left(1-\omega^{-i} \mu_{1} t^{2}\right)\left(1-\mu_{2} t^{2}\right)\left(1-\mu_{2} t^{4}\right)} \\
& =\frac{\left(1-\mu_{1}^{6} t^{12}\right)}{\left(1-\mu_{2} t^{2}\right)\left(1-\mu_{2} t^{4}\right)\left(1-\mu_{1}^{2} t^{4}\right)\left(1-\mu_{1}^{3} t^{6}\right)^{2}} . \\
& \operatorname{HWG}\left(\mathcal{C} / S_{3}\right)=\frac{1}{6}\left(\frac{1}{\left(1-\mu_{1} t^{2}\right)^{2}\left(1-\mu_{2} t^{2}\right)\left(1-\mu_{2} t^{4}\right)}\right. \\
& +\frac{2}{\left(1-\omega \mu_{1} t^{2}\right)\left(1-\omega^{2} \mu_{1} t^{2}\right)\left(1-\mu_{2} t^{2}\right)\left(1-\mu_{2} t^{4}\right)} \\
& \left.+\frac{3}{\left(1-\mu_{1} t^{2}\right)\left(1+\mu_{1} t^{2}\right)\left(1-\mu_{2} t^{2}\right)\left(1+\mu_{2} t^{4}\right)}\right) \\
& =\frac{1-\mu_{1}^{6} \mu_{2}^{2} t^{20}}{\left(1-\mu_{2} t^{2}\right)\left(1-\mu_{1}^{2} t^{4}\right)\left(1-\mu_{1}^{3} t^{6}\right)\left(1-\mu_{2}^{2} t^{8}\right)\left(1-\mu_{1}^{3} \mu_{2} t^{10}\right)} \text {. }
\end{aligned}
$$

This reproduces the results in [19].

Eighth case of table 2. We can apply similar methods to the eighth line of table 2 . The HWG for the minimal nilpotent orbit of $F_{4}$, written in terms of $D_{4}$ fugacities, is $\operatorname{PE}\left[\left(\mu_{1}+\mu_{2}+\mu_{3}+\mu_{4}\right) t^{2}\right]$. The weights $\mu_{1}, \mu_{3}$ and $\mu_{4}$ correspond to the external nodes of the Dynkin diagram. In order to perform the $\mathbb{Z}_{2}^{2}$ quotient, we charge them under the three distinct $\mathbb{Z}_{2}$ subgroups and apply formula (3.70). This way one gets the HWG

$$
\frac{1}{4} \sum_{\epsilon_{1}= \pm 1} \sum_{\epsilon_{2}= \pm 1} \frac{1}{\left(1-\epsilon_{1} \epsilon_{2} \mu_{1} t^{2}\right)\left(1-\mu_{2} t^{2}\right)\left(1-\epsilon_{2} \mu_{3} t^{2}\right)\left(1-\epsilon_{1} \mu_{4} t^{2}\right)}
$$

which evaluates to $\mathrm{PE}\left[\mu_{2} t^{2}+\left(\mu_{1}^{2}+\mu_{3}^{2}+\mu_{4}^{2}\right) t^{4}+\mu_{1} \mu_{3} \mu_{4} t^{6}-\mu_{1}^{2} \mu_{3}^{2} \mu_{4}^{2} t^{12}\right]$. One can check that this is indeed the HWG for the closure of the $\left[3,2^{2}, 1\right]$ orbit of $\mathfrak{s o}(8)$. An alternative way of seeing the same computation relies on the fact that $\mathbb{C}^{3} / \mathbb{Z}_{2}^{2}$ is a weighted hypersurface in $\mathbb{C}^{4}$. 


\subsection{Higgs branch of wreathed quivers}

In this subsection, we turn to the Higgs branch of wreathed quivers. This is in contrast with the rest of the paper, which focuses on the Coulomb branch of the $3 d \mathcal{N}=4$ theories, but it serves several purposes. First, it demonstrates that wreathed quivers do indeed provide a well-defined hyper-Kähler quotient, which can be associated with a gauge theory whose gauge group is disconnected. Secondly, we explain how to compute the Hilbert series of such quivers, using an averaging procedure. Finally, it allows the study of the geometric action of wreathing on the Higgs branch and contrasts it with the parallel action on the Coulomb branch.

We focus on a simple but rich example, the affine $D_{4}$ quiver, and compute the Higgs branch of all the wreathed quivers that appear in figure 4 . Let $\Gamma$ be a subgroup of $S_{4}$. We consider the wreathed quiver defined by this group acting on the four $\mathrm{U}(1)$ nodes. This produces (when $\Gamma$ is non-trivial) a disconnected gauge group, as follows directly from the definition (3.1). Disconnected gauge groups have been considered in the context of the plethystic program in [33], where groups were extended by outer automorphisms, following a formula of Wendt [34]. Here the context is different but the techniques spelled out in [33] apply. In fact, the case considered here is particularly easy to handle because the groups which are being wreathed are all U(1) groups, therefore the Haar measure is not modified. We pick fugacities $z_{i}(i=1,2,3,4)$ for the $\mathrm{U}(1)$ factors and fugacity $y$ for the $\mathrm{U}(2)$ factor (after ungauging a diagonal $\mathrm{U}(1)$ ). It follows that the Higgs branch Hilbert series is obtained via a Molien-Weyl integral which is written explicitly as

$$
\operatorname{HS}_{\Gamma}^{\mathcal{H}}(t)=\frac{1}{|\Gamma|} \sum_{\gamma \in \Gamma} \int_{z_{i}, y} \mathrm{~d} \mu\left(z_{i}, y\right) \mathcal{F}^{b}\left(z_{i}, y, t, \gamma\right),
$$

where the measure is

$$
\mathrm{d} \mu\left(z_{i}, y\right)=\frac{\mathrm{d} z_{1}}{2 \pi i z_{1}} \frac{\mathrm{d} z_{2}}{2 \pi i z_{2}} \frac{\mathrm{d} z_{3}}{2 \pi i z_{3}} \frac{\mathrm{d} z_{4}}{2 \pi i z_{4}} \frac{\left(1-y^{2}\right) \mathrm{d} y}{2 \pi i y}
$$

and

$\mathcal{F}^{b}\left(z_{i}, y, t, \gamma\right)=\frac{\operatorname{det}\left(\mathbf{1}_{4}-\gamma t^{2}\right)\left(1-t^{2}\right)\left(1-t^{2} y^{2}\right)\left(1-t^{2} y^{-2}\right)}{\operatorname{det}\left(\mathbf{1}_{4}-\gamma t y D\right) \operatorname{det}\left(\mathbf{1}_{4}-\gamma t y^{-1} D\right) \operatorname{det}\left(\mathbf{1}_{4}-\gamma t y D^{-1}\right) \operatorname{det}\left(\mathbf{1}_{4}-\gamma t y^{-1} D^{-1}\right)}$

with $D$ the diagonal matrix $\operatorname{Diag}\left(z_{1}, z_{2}, z_{3}, z_{4}\right)$. The integral over the $z_{i}$ and $y$ fugacities are performed over the contours $\left|z_{i}\right|=|y|=1$. Note that (3.78) makes it manifest that $\gamma \in \Gamma$ can be considered as a discrete fugacity for the disconnected gauge group $\mathrm{U}(1)<\Gamma$. The integrals (3.78) are readily evaluated for each of the 11 subgroups of $S_{4}$, and the resulting Hilbert series are presented in figure 5 .

We make a few comments on the results. First, the Hilbert series coincide with those of Du Val singularities $\mathbb{C}^{2} / J$, with $J$ a finite subgroup of $\mathrm{SU}(2)$, of ADE type. Specifically, four instances occur, namely $J=D_{4}, D_{6}, E_{6}, E_{7}$, that can be identified using the degrees of invariants of the corresponding groups. In particular, this shows that the quaternionic dimension of the Higgs branches of all these quivers is 1 . 


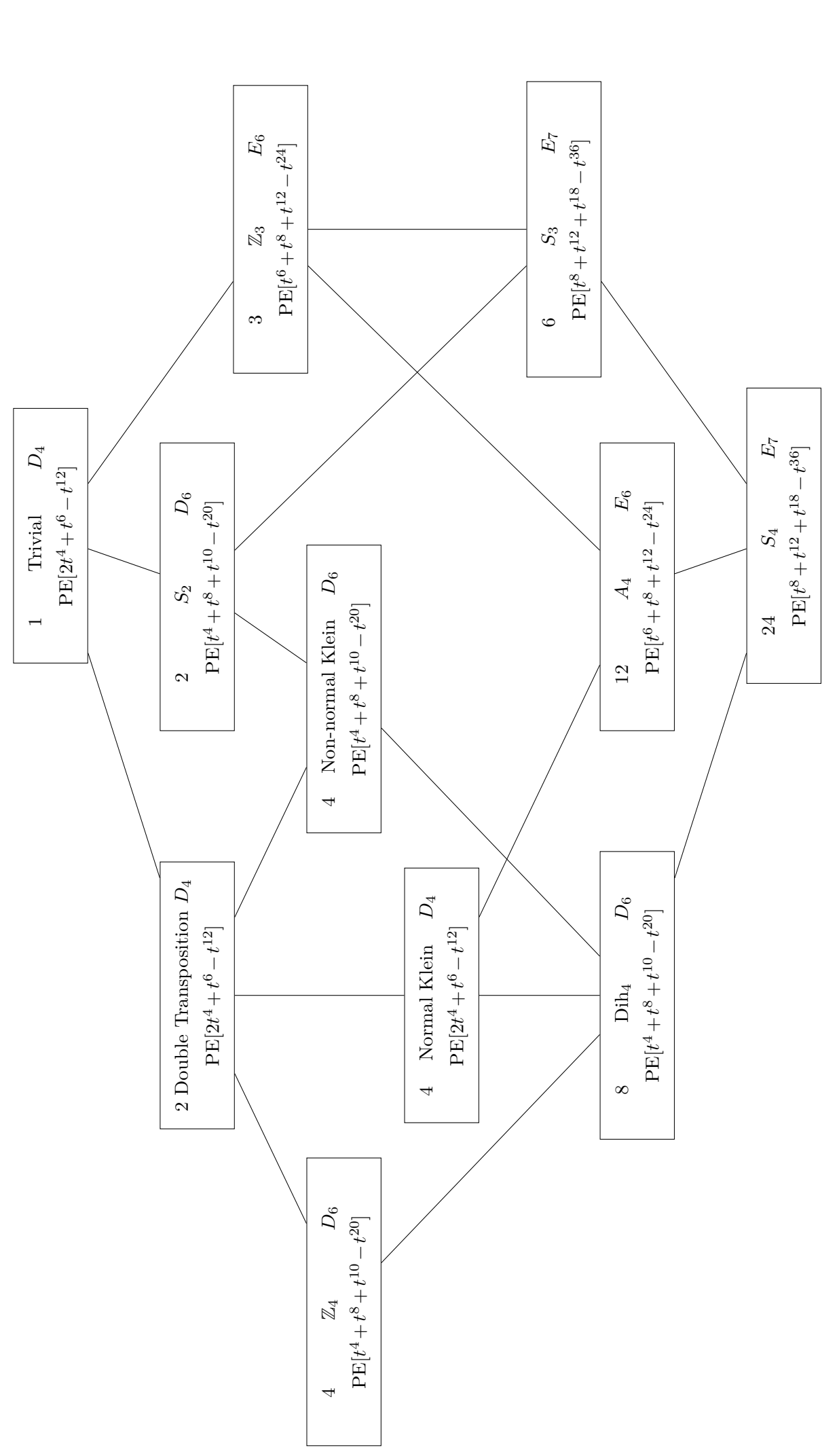

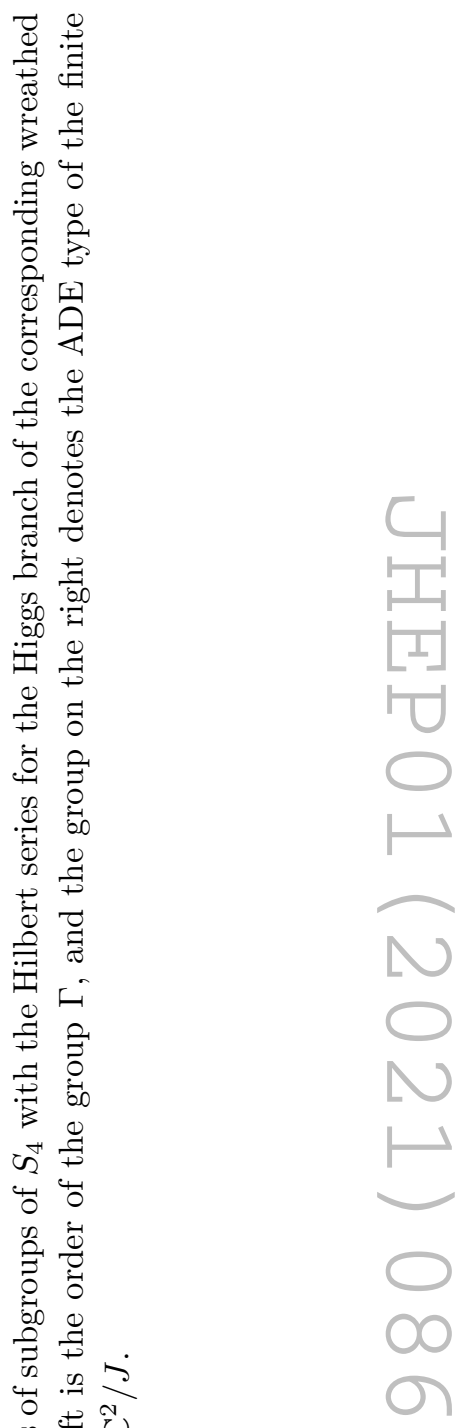

कु

용

矢 专

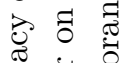

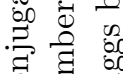

응

$\exists \Xi$

焉论

명 형

$50 \%$ is

: $\exists$ 워

蛇

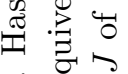

10 0 요

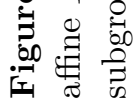


Gauge invariant operators. As a check of the computations presented in figure 5, we briefly show how the same results can be obtained from a counting of invariant operators. We call $A_{i}$ and $B_{i}$ the scalars in the chiral multiplets transforming as bifundamentals of $\mathrm{U}(2)$ and $\mathrm{U}(1)_{i}$, for $i=1,2,3,4, A_{i}$ being a column vector and $B_{i}$ being a row vector. For simplicity, we ungauge one of the $\mathrm{U}(1)$ groups, say $\mathrm{U}(1)_{4}$, and study the action of the wreath product by a subgroup $\Gamma$ of $S_{3}$ permuting the three remaining U(1) gauge groups.

The F-term equations on $\mathrm{U}(1)_{i}$ are

$$
\text { For } i=1,2,3, \quad B_{i} A_{i}=0 .
$$

The F-term equations on the $\mathrm{U}(2)$ group are

$$
\sum_{i=1}^{4} A_{i} B_{i}=0 .
$$

Taking the trace of (3.82) and combining with (3.81) we obtain

$$
B_{4} A_{4}=0 \text {. }
$$

Gauge invariants are paths in the quiver of the form $B_{4} \alpha_{i_{1}} \cdots \alpha_{i_{r}} A_{4}$ subject to the relations above, using the shorthand notation $\alpha_{i}=A_{i} B_{i}$. An irreducible gauge invariant is one that can not be written as a product of other non-trivial gauge invariants, so it can be written $B_{4} \alpha_{i_{1}} \cdots \alpha_{i_{r}} A_{4}$ where the indices can not take the value 4 . The F-term relations imply that

$$
\alpha_{i} \alpha_{i}=0 \text { and } \sum_{i=1}^{4} \alpha_{i}=0 .
$$

In particular an irreducible gauge invariant can not contain three $\alpha_{i}$ 's or more. ${ }^{14}$ So generators of the Higgs branch coordinate ring contain either one or two $\alpha_{i}$ 's. The generators containing one $\alpha_{i}$ are $X_{i}=B_{4} \alpha_{i} A_{4}(i=1,2,3)$ subjected to $X_{1}+X_{2}+X_{3}=0$, and transform in the irreducible two-dimensional representations of $S_{3}$. The generators with two $\alpha_{i}$ 's are built from $Y_{i j}=B_{4} \alpha_{i} \alpha_{j} A_{4}$. Note that $Y_{i j}=-Y_{j i}$ and that $Y_{12}=Y_{23}=Y_{31}$, which shows that the $Y_{i j}$ transform in the $\varepsilon$ representation of $S_{3}$. Finally, there is a relation between the two families, for instance in the form

$$
X_{1} X_{2} X_{3}=B_{4} \alpha_{1} \alpha_{4} \alpha_{2} \alpha_{4} \alpha_{3} A_{4}=B_{4} \alpha_{1} \alpha_{3} \alpha_{2} \alpha_{1} \alpha_{3} A_{4}=-Y_{12}^{2} .
$$

Putting all this together, we obtain the Hilbert series PE[2t $\left.+t^{6}-t^{12}\right]$ for the affine $D_{4}$ quiver (the $X_{i}$ have weight 4 while the $Y_{i j}$ have weight 6 ). To deal with the wreathed quivers, we have to impose the additional gauge invariance under the discrete factor $\Gamma$. The spectrum of operators on the Higgs branch is a subset of the one determined above for trivial $\Gamma$. The results are gathered in table 7 .

\footnotetext{
${ }^{14}$ Consider for instance $B_{4} \alpha_{i} \alpha_{j} \alpha_{k} A_{4}$ with $i \neq j, j \neq k$ and $i, j, k \neq 4$. If $i \neq k$ then one finds $B_{4} \alpha_{i} \alpha_{j} \alpha_{k} A_{4}=-B_{4} \alpha_{i}\left(\alpha_{i}+\alpha_{k}+\alpha_{4}\right) \alpha_{k} A_{4}=B_{4} \alpha_{i} \alpha_{4} \alpha_{k} A_{4}=\left(B_{4} \alpha_{i} A_{4}\right)\left(B_{4} \alpha_{k} A_{4}\right) . \quad$ If $i=k$ then $B_{4} \alpha_{i} \alpha_{j} \alpha_{i} A_{4}=-B_{4} \alpha_{i} \alpha_{j} \alpha_{l} A_{4}$ with $l \neq i, j, 4$ and we're back in the previous case.
} 


\begin{tabular}{|c|c|c|c|}
\hline Group $\Gamma$ & Generators & Relation & Geometry \\
\hline$S_{1}$ & $\begin{array}{ll}t^{4}: & x=X_{1} \\
t^{4}: & y=X_{2} \\
t^{6}: & z=Y_{12}\end{array}$ & $x y(x+y)=z^{2}$ & $\mathbb{C}^{2} / D_{4}$ \\
\hline$S_{2}$ & $\begin{array}{cc}t^{4}: & x=X_{1}+X_{2} \\
t^{8}: & y=X_{1} X_{2} \\
t^{10}: & z=Y_{12}\left(X_{1}-X_{2}\right)\end{array}$ & $x y\left(x^{2}-4 y\right)=z^{2}$ & $\mathbb{C}^{2} / D_{6}$ \\
\hline $\mathbb{Z}_{3}$ & $\begin{array}{cc}t^{6}: & x=Y_{12} \\
t^{8}: & y=X_{1}^{2}+X_{1} X_{2}+X_{2}^{2} \\
t^{12}: & z=\left(X_{1}-X_{2}\right)\left(2 X_{1}+X_{2}\right)\left(X_{1}+2 X_{2}\right)\end{array}$ & $27 x^{4}-4 y^{3}+z^{2}=0$ & $\mathbb{C}^{2} / E_{6}$ \\
\hline$S_{3}$ & $\begin{array}{cc}t^{8}: & x=X_{1}^{2}+X_{1} X_{2}+X_{2}^{2} \\
t^{12}: & y=Y_{12}^{2} \\
t^{18}: & z=Y_{12}\left(X_{1}-X_{2}\right)\left(2 X_{1}+X_{2}\right)\left(X_{1}+2 X_{2}\right)\end{array}$ & $-4 x^{3} y+27 y^{3}+z^{2}=0$ & $\mathbb{C}^{2} / E_{7}$ \\
\hline
\end{tabular}

Table 7. Generators and relations for operators on the Higgs branch of the affine $D_{4}$ quiver wreathed by subgroups of $S_{3}$.

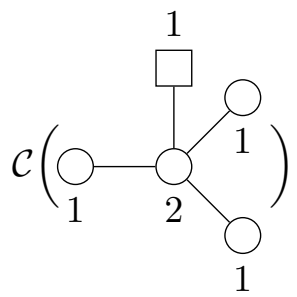

(a)

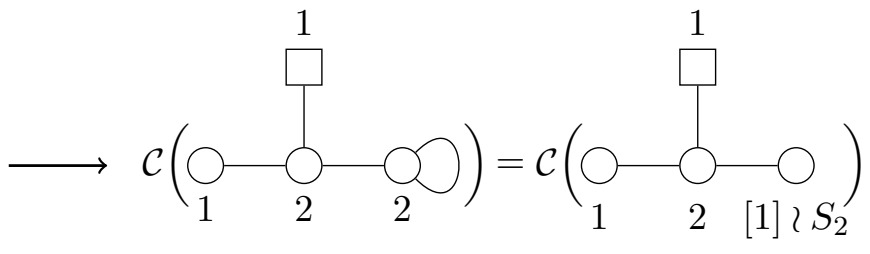

(b) (c)

Figure 6. The Coulomb branch of the $D_{4}$ quiver (left) is orbifolded by an $S_{2}$ action into a Coulomb branch shared by two distinct quivers.

Comparison with adjoint matter. Consider the case depicted in figure 6 . In $[5,6]$ it was pointed out that the Coulomb branch of the quiver (b) is an orbifold of the Coulomb branch of the quiver (a). We have argued that the Coulomb branch of the wreathed quiver (c) is also that very same orbifold. Let's look at the Higgs branch of quiver (b).

The (quaternionic) dimension of the Higgs branch, when there is complete Higgsing, which is the case here, is equal to the number of matter multiplets minus the number of gauge multiplets. The $D_{4}$ quiver therefore has $\operatorname{dim} \mathcal{H}_{D_{4}}=(4 \cdot 2 \cdot 1)-\left(3 \cdot 1+2^{2}\right)=1$, as do all the wreathed quivers. The quiver (b) has a Higgs branch of quaternionic dimension $\operatorname{dim} \mathcal{H}_{\text {loop }}^{\mathrm{U}(2)}=\left(2 \cdot 2 \cdot 1+2 \cdot 2+2^{2}\right)-\left(1+2 \cdot 2^{2}\right)=3$, of which one dimension is a free factor $\mathbb{H}$ from the trivial factor in the adjoint loop. We can be more precise and compute the 


\begin{tabular}{|c|c|c|c|}
\hline Coulomb Quiver & Discretely Gauged & Higgs Quiver & Discretely Gauged \\
\hline $\mathcal{C}=\overline{\min D_{4}}$ & $\mathcal{C}=\overline{\mathrm{n} \cdot \min B_{3}}=\frac{\frac{\min D_{4}}{S_{2}}}{1}$ & $\mathcal{H}=\overline{\min D_{4}}$ & $\mathcal{H}=\overline{\mathrm{n} . \min B_{3}}=\frac{\overline{\min D_{4}}}{S_{2}}$ \\
\hline
\end{tabular}

Table 8. Illustration of the relation between i) discrete gauging's effects on the Coulomb branch and ii) discrete gauging's effects on the Higgs branch of a corresponding electric quiver.

Hilbert series using the hyper-Kähler quotient, finding

$$
\mathrm{PE}[2 t] \mathrm{PE}\left[3 t^{2}+2 t^{5}-t^{12}\right]
$$

The first term comes from a free contribution $\mathbb{H}$ which can be discarded. The second term can be identified as the Hilbert series for an intersection of a Slodowy slice and the nilpotent cone in the $C_{3}$ algebra, namely the transverse slice between the maximal orbit (of dimension 9) and the $\mathcal{O}_{\left[4,1^{2}\right]}$ orbit of dimension 7, see table 12 in [35] (labelled [210] therein). The global symmetry on this space is $\operatorname{Sp}(1)$ under which the generators of the chiral ring transform in the [2] and the [1] representations, respectively. This space makes a rare occurrence of a symplectic singularity which is also a hypersurface in $\mathbb{C}^{5}$. In fact it has been suggested that all hypersurface symplectic singularities of dimension 2 are intersections of Slodowy slices of the nilpotent orbit $\mathcal{O}_{\left[2 n-2,1^{2}\right]}$ and the nilpotent cone in $C_{n}$ [36]. This family appears in the context of trivertex theories where the rank of $C_{n}$ is interpreted as the genus of a Riemann surface ( $A_{1}$ class $\mathrm{S}$ theory on a Riemann surface of genus $n$ and one puncture). See section 7.2 of [37] and equation (7.12) for the hypersurface equation. The same family also appears as a Coulomb branch of the mirror quiver in the work of [38] where the identification as a transverse slice is made, as well as an explicit form of the hypersurface equation. The Hasse diagram is

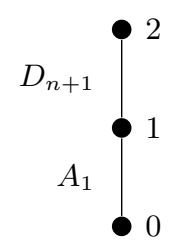

In summary, the two quivers on the right of figure 6 share the same Coulomb branch, but only the wreathed quiver's Higgs branch shares the original quiver's Higgs branch dimension, as one would expect from discrete gauging.

\subsection{Mirror symmetry and discrete gauging}

Many $3 d \mathcal{N}=4$ quiver theories admit a dual description as a theory whose Higgs branch is the original's Coulomb branch and vice versa; this property is known as $3 d$ mirror 

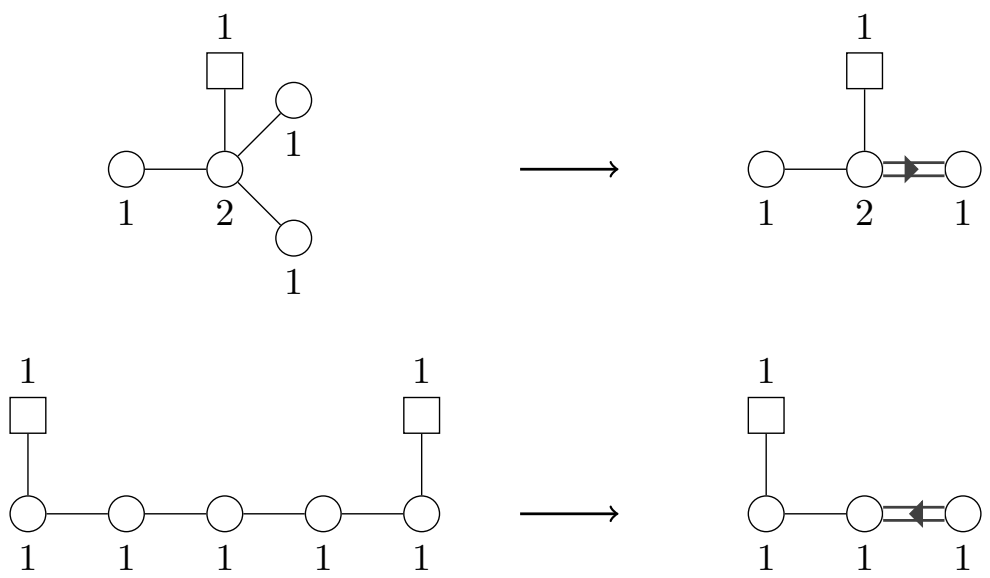

Figure 7. Quivers on the left fold into quivers on the right.

symmetry [39-41] and is a consequence of S-duality for theories with brane realisations. One should therefore expect to be able to find the mirror dual of discrete gauging. As it turns out, it is already known.

Let us consider the paradigmatic case of quivers in table 8. The Coulomb branch of the quiver in the first column is the minimal nilpotent orbit of $D_{4}$. Its dual is depicted in the third column of the same figure; the symmetry of its Higgs branch is the same as the symmetry on the flavor node. Each matter hypermultiplet is coupled to a mass which can be viewed as a background vector multiplet. This vector can in turn be gauged, turning the quiver into the one depicted in the fourth column; such an operation was first reported as "the case $O(1)$ " in [21]. The new gauge node $O(1) \cong \mathbb{Z}_{2} \cong S_{2}$ represents the discrete symmetry of the gauged vector. In this case the gauge group is enlarged. We claim this is the mirror dual of the process covered in the previous section. Somewhat confusingly, both procedures are called discrete gauging ${ }^{15}$ but they act differently. On the left quiver an automorphism is gauged; on the right we gauge a background vector.

If the enhancement of the mirror is discrete, so must be the original's. Moreover, since discrete gauging of background vectors is a genuine action on quiver theories, so is its mirror dual.

\section{Quiver folding}

The next discrete operation allows for a natural interpretation of non-simply laced quivers, which were identified in [1] through the use of the monopole formula. It was already well established [2] that many $A D E$ nilpotent orbits could be recovered as Coulomb branches of unitary quiver theories and that there is a robust connection between choice of quiver and the resulting nilpotent orbit. In particular, the quiver should be balanced and shaped like the desired symmetry algebra's Dynkin diagram. Consequently one might assume that quivers whose Coulomb branches reproduce $B C F G$ nilpotent orbits would resemble the

\footnotetext{
${ }^{15}$ We are not aware of a physics reference for discrete gauging on the Higgs branch but believe it to be fairly well known among physicists interested in Higgs branches of quiver gauge theories.
} 
non-simply laced $B C F G$ Dynkin diagrams. Ref. [1] conjectured a minimal modification to the monopole formula which reflected the enigmatic multiple link, checking against earlier tentative results of [42] on $F_{4}$ and $G_{2}$ spaces. Although the conjecture was highly successful in its goal, giving support to the existence of non-simply laced quivers and allowing further study [43], precise details of multiple links remained elusive. ${ }^{16}$ They have made an appearance in the study of little string theory [44] or gauge-vortex duality [45] and $W$-algebras associated to them were studied in [46]. A mathematical treatment of folding and Coulomb branches of non-simply laced quivers was recently provided in [3]. Some of the phenomena in $[47,48]$ can be reinterpreted as folding the five-dimensional theories' magnetic quivers [49].

In this section we show (using an alternative approach to [3]) that the multiple link can be interpreted as the result of quiver folding; see figure 7 for examples. We first utilise abelianisation to show that Coulomb branches of $A_{2 n-1}\left(D_{n+1}\right)$ quivers fold into spaces with $C_{n}\left(B_{n}\right)$ symmetry and derive the effects of folding on the monopole formula. We then reinterpret folding as an action on the quiver itself, showing that it produces non-simply laced quivers; in particular, our analysis of the monopole formula on examples reproduces the form in $[1,3]$.

Note that the examples below focus on nilpotent orbit quivers only because they are most easily studied using tools we have developed. We expect folding to be a completely general operation. For example, the quiver of section 4.1.2 in [50] folds into the quiver in (7.1) of [19] as can be guessed by mapping $\mu_{2 N-i} \mapsto \mu_{i}$ for $i<N$ in the former's HWG and comparing to the HWG of the latter quiver.

\subsection{Action on the Coulomb branch}

Although one can fold a quiver directly, the operation can also be performed on a discretely gauged Coulomb branch. The prerequisites for folding and discrete gauging are identical: a quiver with an automorphism. We start yet again with the example of a $D_{4}$ quiver in the bottom left of figure 7 . Recall that in (3.20) the discretely gauged quiver's operator $\tilde{e}_{\langle 0,0,1\rangle}$ is defined as $e_{\langle 0,0,1,0\rangle}+e_{\langle 0,0,0,1\rangle}=u_{3}^{+}+u_{4}^{+}$. A space is folded by restricting it to the subspace fixed under the action of the symmetry, which in this case generates the constraints $u_{3}^{+}=u_{4}^{+}$ as well as $\varphi_{3}=\varphi_{4}$ and so on; we denote this space $\hat{\mathcal{C}}$ and in general use hats to denote variables on the folded space. Note that mass parameters must be set to identical values across folded legs; sometimes this removes all independent mass parameters but one and, as a result, even though the original space is mass-deformable, the folded space is not.

As long as we stay on $\hat{\mathcal{C}}$ there is no more need to track each individual wreathed variable. To reduce to a minimal necessary set we introduce the folding map

$$
\begin{aligned}
F\left(x_{i, a}\right) & =\frac{\hat{x}_{I, a}}{\#_{i}} \\
F(x+y) & =F(x)+F(y) \\
F\left(c x^{m} y^{n}\right) & =c F(x)^{m} F(y)^{n}
\end{aligned}
$$

\footnotetext{
${ }^{16}$ According to [1], Jan Troost suggested that quivers of this type might be understood as folded simply laced quivers, an idea that ultimately finds validation in [3] and our results.
} 
where the multiplicity $\#_{i}$ denotes the number of nodes that fold onto the same node as node $i, x$ and $y$ are arbitrary operators, $c$ is a complex number and $I=\min _{j}\{j: \pi(j)=\pi(i)\}$. In particular, $F\left(u_{3}^{+}\right)=F\left(u_{4}^{+}\right)=\frac{\hat{u}_{3}^{+}}{2}$. As a result, $F\left(\tilde{e}_{\langle 0,0,1\rangle}\right)=\hat{u}_{3}^{+}=\hat{e}_{\langle 0,0,1\rangle}$.

The folding map has a simple interpretation. Abelianised variables of the initial, unfolded quiver, partition into orbits of the automorphism. The folding map merely sets every single variable in that orbit to the same value; for convenience, basic abelianised variables are normalised by node multiplicity. In other words, the folded Coulomb branch is a restricted subspace of the discretely gauged quiver's Coulomb branch.

While abelianised variables fold in a completely trivial manner, composite operators are more interesting. For example, let's fold the operator in (3.20):

$$
\begin{aligned}
\hat{e}_{\langle 0,1,2\rangle} & =F\left(\tilde{e}_{\langle 0,1,2\rangle}\right) \\
& =F\left(e_{\langle 0,1,1,1\rangle}\right) \\
& =\sum_{a=1,2} \frac{F\left(u_{2, a}^{+} u_{3}^{+} u_{4}^{+}\right)}{F\left(\varphi_{2, a}-\varphi_{3}\right) F\left(\varphi_{2, a}-\varphi_{4}\right)} \\
& =\sum_{a=1,2} \frac{\hat{u}_{2, a}^{+} \hat{u}_{3}^{+2} / 4}{\left(\varphi_{2, a}-\hat{\varphi}_{3} / 2\right)^{2}} \\
& =\sum_{a=1,2} \frac{\hat{u}_{2, a}^{+} \hat{u}_{3}^{+2}}{\left(2 \varphi_{2, a}-\hat{\varphi}_{3}\right)^{2}}
\end{aligned}
$$

If the folded space is to retain the original's hyper-Kähler property, the symplectic property in particular must be preserved and the Poisson brackets on the folded space must close. In other words for any $\hat{f}, \hat{g} \in \mathbb{C}[\hat{\mathcal{C}}]$ we require $\{\hat{f}, \hat{g}\} \in \mathbb{C}[\hat{\mathcal{C}}]$, ie. $\{\hat{f}, \hat{g}\}=\pi(\{\hat{f}, \hat{g}\})$. It is enough to show that generators $\hat{x}_{i, a}$ of the Poisson algebra satisfy this property:

$$
\left\{x_{i, a}, x_{j, b}\right\}=F\left(x_{k, c}\right)=F\left(x_{\pi(k), c}\right)=\pi\left(F\left(x_{k, c}\right)\right)=\pi\left(\left\{x_{i, a}, x_{j, b}\right\}\right)
$$

where we restrict to the folding locus

$$
x_{i, a}=x_{\pi(i), a}, \forall \pi \in \Gamma \subset \text { Aut } Q .
$$

where $\Gamma$ is the subgroup by whose action we fold.

So we have in our hands two pieces: a "folded" subspace (with its coordinate ring) and a Poisson bracket on this space. If we assume that the complex structures also properly restrict to the subspace, we have a new hyper-Kähler space to study. What is it? What is its symmetry?

Now we re-establish contact with discrete gauging. For $\tilde{\mathcal{O}}_{i} \in \mathbb{C}[\tilde{\mathcal{C}}]_{2}$ and $\hat{\mathcal{O}}_{i} \in \mathbb{C}[\hat{\mathcal{C}}]_{2}$, we have

$$
\left\{\tilde{\mathcal{O}}_{i}, \tilde{\mathcal{O}}_{j}\right\}=\sum_{k} c_{i j}{ }^{k} \tilde{\mathcal{O}}_{k}
$$

and therefore the relations in particular hold on the automorphism's fixed point, which is the folded subspace:

$$
\left\{\hat{\mathcal{O}}_{i}, \hat{\mathcal{O}}_{j}\right\}=\sum_{k} c_{i j}{ }^{k} \hat{\mathcal{O}}_{k}
$$


Therefore, unless some folded $\hat{\mathcal{O}}_{k}$ identically vanish, the two algebras have identical structure constants and are in fact isomorphic as Lie algebras. A simple proof in appendix B shows that $\hat{\mathcal{O}}_{k}$ is not 0 everywhere on the folded space so we conclude that folded spaces have the same continuous symmetries as their discretely gauged counterparts.

In particular, a $A_{2 n-1}\left(D_{n+1}\right)$ quiver's Coulomb branch folds into a $C_{n}\left(B_{n}\right)$-symmetric space of strictly lower dimension and the minimal nilpotent orbit of $D_{4}$ folds into the minimal nilpotent orbit of $B_{3}$. Of course this space is just the Coulomb branch of a nonsimply laced quiver, and we claim this is no coincidence: although we have so far only explored folding as an action on the Coulomb branch, we conjecture it is in fact merely one facet of an action on the quiver theory and that all non-simply laced quivers can be understood as folded simply laced quivers.

As was hinted in section 2.1, in some special cases a $B_{3}$ non-simply laced quiver, eg. the bottom right quiver in figure 7 , can fold into $G_{2}$ despite the lack of an obvious symmetry. There is one major difference however: multiplicities are assigned in a more involved manner. As a prerequisite, the "short root" (i.e. third) gauge node must have the same rank and number of flavors as the "vector root" (i.e. first node). We can unfold the $B_{3}$ quiver into a $D_{4}$ shape by simply reversing the folding procedure. Let us denote the variables of that quiver's Coulomb branch e.g. $\varphi_{i}^{D_{4}}$, with $\varphi_{i}^{B_{3}}$ and $\varphi_{i}^{G_{2}}$ the partially and fully folded counterparts. Then at the $D_{4} \rightarrow G_{2}$ folding locus the following holds:

$$
\varphi_{1}^{D_{4}}=\varphi_{3}^{D_{4}}=\varphi_{4}^{D_{4}}=\varphi_{1}^{B_{3}}=\frac{\varphi_{3}^{B_{3}}}{2}=\frac{\varphi_{1}^{G_{2}}}{3}
$$

So the $B_{3}$ quiver can fold to $G_{2}$ as if $\mu_{1}=3$ and $\mu_{3}=\frac{3}{2}$.

\subsection{Monopole formula: examples}

To show that folded quivers become non-simply laced, we compute two explicit examples and conjecture that the pattern generalises.

\subsection{1 $\min A_{3} \rightarrow \min C_{2}$}

The first check will be done on quivers in table 9 by folding two U(1) nodes.

Let $\mathrm{HS}^{A}$ and $\mathrm{HS}^{C}$ be the Hilbert series of the initial and folded quivers, respectively:

$$
\begin{aligned}
\operatorname{HS}^{A}(t, x, y, z) & =\frac{1}{\left(1-t^{2}\right)^{3}} \sum_{q_{1}, q_{2}, q_{3} \in \mathbb{Z}} t^{\left|q_{1}\right|+\left|q_{1}-q_{2}\right|+\left|q_{2}-q_{3}\right|+\left|q_{3}\right|}(x y)^{q_{1}}\left(\frac{x}{y}\right)^{q_{3}} z^{q_{2}} \\
\mathrm{HS}^{C}(t, x, z) & =\frac{1}{\left(1-t^{2}\right)^{2}} \sum_{r_{1}, r_{2} \in \mathbb{Z}} t^{\left|r_{1}\right|+\left|r_{1}-2 r_{2}\right|} x^{r_{1}} z^{r_{2}} .
\end{aligned}
$$

The unrefined Hilbert series are:

$$
\begin{aligned}
\mathrm{HS}^{A}(t, 1,1,1) & =\frac{\left(1+t^{2}\right)\left(1+8 t^{2}+t^{4}\right)}{\left(1-t^{2}\right)^{6}} \\
\mathrm{HS}^{C}(t, 1,1) & =\frac{1+6 t^{2}+t^{4}}{\left(1-t^{2}\right)^{4}}
\end{aligned}
$$


Note the unusual fugacity $y$ in $\mathrm{HS}^{A}$ which is crucial in the following calculations. By comparison with known Hilbert series, we find that the two Coulomb branches are the (closures of the) minimal nilpotent orbits of $A_{3}$ and $C_{2}$.

We will now derive the action $\mathrm{HS}^{A} \rightarrow \mathrm{HS}^{C}$ in two steps.

At the level of bare monopole operators, many become duplicate. For example, $\left(u_{1}^{+}\right)^{2}$, $u_{1}^{+} u_{3}^{+}$and $\left(u_{3}^{+}\right)^{2}$ all fold to $\frac{\left(\hat{u}_{1}^{+}\right)^{2}}{4}$. More generally, a bare monopole monomial in the $A_{3}$ theory can be expressed (not necessarily uniquely) as a product of generators

$$
\mathcal{O}_{q_{1}, q_{2}, q_{3}}=\prod_{i} e_{\left\langle q_{1}^{i}, q_{2}^{i}, q_{3}^{i}\right\rangle}
$$

where $q_{j}=\sum_{i} q_{j}^{i}$. Note that $\left|q_{1}^{i}-q_{3}^{i}\right| \in\{0,1\}$. The $S_{2}$ symmetry exchanges $e_{\left\langle q_{1}^{i}, q_{2}^{i}, q_{3}^{i}\right\rangle} \leftrightarrow$ $e_{\left\langle q_{3}^{i}, q_{2}^{i}, q_{1}^{i}\right\rangle}$ and acting with it on any number of operators in the product produces a monopole in the $A_{3}$ theory which folds to the exact same monopole in the $C_{2}$ theory. "Flipping" a single operator in this way leaves $q_{1}$ and $q_{3}$ unchanged or changes both by \pm 1 with opposite signs so that $q_{1}+q_{3}$ is preserved. Sequential action on all the monopoles in the product produces $\mathcal{O}_{q_{3}, q_{2}, q_{1}}$. It follows that in this chain of flips there is an operator $\mathcal{O}_{\frac{q_{1}+q_{3}}{2}, q_{2}, \frac{q_{1}+q_{3}}{2}}$ or $\mathcal{O}_{\frac{q_{1}+q_{3}+1}{2}}, q_{2}, \frac{q_{1}+q_{3}-1}{2}$, depending on the parity of $q_{1}+q_{3}$. Since all operators in the chain fold to the same operator, the $C_{2}$ monopole formula better count precisely one of them. We will pick either $\mathcal{O}_{\frac{q_{1}+q_{3}}{2}, q_{2}}, \frac{q_{1}+q_{3}}{2}$ or $\mathcal{O}_{\frac{q_{1}+q_{3}+1}{2}, q_{2}, \frac{q_{1}+q_{3}-1}{2}}$ (of which precisely one exists), which translates to selecting only monopoles with $q_{1}=q_{3}$ or $q_{1}=q_{3}+1$, respectively.

To accomplish this we must extract only the terms constant and linear in $y$, as can be seen from (4.10): terms constant in $y$ come from the charge sublattice $q_{1}=q_{3}$ while linear terms all satisfy $q_{1}=q_{3}+1$. To set up later generalisation we further slightly modify the prescription to an equivalent form: we will extract every operator at order $y^{0}$ and average over operators at order $y$ and $y^{-1}$.

The second step corrects for scalar dressing: one extraneous scalar field must be removed since $\varphi_{1}=\varphi_{3}=\frac{\hat{\varphi}_{1}}{2}$. We need only multiply the entire expression with $1-t^{2}$ to remove the newly duplicate $\mathrm{U}(1)$ dressing factor $\frac{1}{1-t^{2}}$.

We conjecture that these two modifications are sufficient to represent the action of folding on the Hilbert series.

To implement them, we multiply the (unsummed) monopole formula by the kernel $\frac{1}{2 \pi i y}\left(1+\frac{1}{2}\left(y+\frac{1}{y}\right)\right)$ and integrate around $y=0$, picking up the desired contributions by the residue theorem. Finally we multiply by the scalar factor $\left(1-t^{2}\right)$ :

$$
\operatorname{HS}^{C}(t, x, z)=\left(1-t^{2}\right) \oint \frac{\mathrm{d} y}{2 \pi i y}\left(1+\frac{1}{2}\left(y+\frac{1}{y}\right)\right) \operatorname{HS}^{A}(t, x, y, z)
$$

And indeed:

$$
\begin{aligned}
\text { R.h.s. } & =\frac{1}{2}\left(1-t^{2}\right)^{-2} \oint \frac{\mathrm{d} y}{2 \pi i y}\left(y+\frac{1}{y}+2\right) \sum_{q_{1}, q_{2}, q_{3} \in \mathbb{Z}} t^{\left|q_{1}\right|+\left|q_{1}-q_{2}\right|+\left|q_{2}-q_{3}\right|+\left|q_{3}\right|}(x y)^{q_{1}}\left(\frac{x}{y}\right)^{q_{3}} z^{q_{2}} \\
& =\frac{1}{2}\left(1-t^{2}\right)^{-2} \oint \frac{\mathrm{d} y}{2 \pi i y} \sum_{q_{1}, q_{2}, q_{3} \in \mathbb{Z}} t^{\left|q_{1}\right|+\left|q_{1}-q_{2}\right|+\left|q_{2}-q_{3}\right|+\left|q_{3}\right|} x^{q_{1}+q_{3}} y^{q_{1}-q_{3}-1} z^{q_{2}}
\end{aligned}
$$




$$
\begin{aligned}
& +\frac{1}{2}\left(1-t^{2}\right)^{-2} \oint \frac{\mathrm{d} y}{2 \pi i y} \sum_{q_{1}, q_{2}, q_{3} \in \mathbb{Z}} t^{\left|q_{1}\right|+\left|q_{1}-q_{2}\right|+\left|q_{2}-q_{3}\right|+\left|q_{3}\right|} x^{q_{1}+q_{3}} y^{q_{1}-q_{3}+1} z^{q_{2}} \\
& +\left(1-t^{2}\right)^{-2} \oint \frac{\mathrm{d} y}{2 \pi i y} \sum_{q_{1}, q_{2}, q_{3} \in \mathbb{Z}} t^{\left|q_{1}\right|+\left|q_{1}-q_{2}\right|+\left|q_{2}-q_{3}\right|+\left|q_{3}\right|} x^{q_{1}+q_{3}} y^{q_{1}-q_{3}} z^{q_{2}} \\
= & \frac{1}{2}\left(1-t^{2}\right)^{-2} \sum_{r_{2} \in \mathbb{Z}, r_{1} \in(2 \mathbb{Z}+1)} t^{\left|\left(r_{1}+1\right) / 2\right|+\left|\left(r_{1}+1\right) / 2-r_{2}\right|+\left|r_{2}-\left(r_{1}-1\right) / 2\right|+\left|\left(r_{1}-1\right) / 2\right|} x^{r_{1}} z^{r_{2}} \\
& +\frac{1}{2}\left(1-t^{2}\right)^{-2} \sum_{r_{2} \in \mathbb{Z}, r_{1} \in(2 \mathbb{Z}+1)} t^{\left|\left(r_{1}-1\right) / 2\right|+\left|\left(r_{1}-1\right) / 2-r_{2}\right|+\left|r_{2}-\left(r_{1}+1\right) / 2\right|+\left|\left(r_{1}+1\right) / 2\right|} x^{r_{1}} z^{r_{2}} \\
& +\left(1-t^{2}\right)^{-2} \sum_{r_{2} \in \mathbb{Z}, r_{1} \in(2 \mathbb{Z})} t^{\left|r_{1} / 2\right|+\left|r_{1} / 2-r_{2}\right|+\left|r_{2}-r_{1} / 2\right|+\left|r_{1} / 2\right|} x^{r_{1}} z^{r_{2}} \\
& \left(1-t^{2}\right)^{-2} \sum_{r_{2} \in \mathbb{Z}, r_{1} \in(2 \mathbb{Z}+1)} t^{\left|\left(r_{1}+1\right) / 2\right|+\left|\left(r_{1}+1\right) / 2-r_{2}\right|+\left|r_{2}-\left(r_{1}-1\right) / 2\right|+\left|\left(r_{1}-1\right) / 2\right|} x^{r_{1}} z^{r_{2}} \\
& +\left(1-t^{2}\right)^{-2} \sum_{r_{2} \in \mathbb{Z}, r_{1} \in(2 \mathbb{Z})} t^{\left|r_{1}\right|+\left|r_{1}-2 r_{2}\right|} x^{r_{1}} z^{r_{2}} \\
= & \left(1-t^{2}\right)^{-2} \sum_{r_{1}, r_{2} \in \mathbb{Z}} t^{\left|r_{1}\right|+\left|r_{1}-2 r_{2}\right|} x^{r_{1}} z^{r_{2}}
\end{aligned}
$$

In particular note the appearance of 2 in $\left|r_{1}-2 r_{2}\right|$, the novel feature in non-simply laced quivers' monopole formulas.

\subsection{2 $\min D_{4} \rightarrow \min G_{2}$}

We now look at the folding of three $\mathrm{U}(1)$ gauge nodes of the $D_{4}$ minimal nilpotent orbit quiver. We again assign fugacities to the nodes: call $z$ the fugacity for the $\mathrm{U}(2)$ node, and $x y_{1}, x \frac{y_{2}}{y_{1}}, x \frac{1}{y_{2}}$ the fugacities for the three $\mathrm{U}(1)$ nodes. This parametrisation is chosen so that folding corresponds to an integration over the $y_{i}$, which have an $A_{2}$ symmetry. Note that this prescription generalises the previous example, where the "folding fugacity" appeared as $y$ and $y^{-1}$, which are related by an $A_{1}$ symmetry.

The folding equation becomes

$$
\operatorname{HS}^{G_{2}}(t, x, z)=\left(1-t^{2}\right)^{2} \oint \frac{\mathrm{d} y_{1}}{2 \pi i y_{1}} \frac{\mathrm{d} y_{2}}{2 \pi i y_{2}} f\left(y_{1}, y_{2}\right) H^{D_{4}}\left(t, x, y_{1}, y_{2}, z\right)
$$

with

$$
f\left(y_{1}, y_{2}\right)=1+\frac{1}{3}\left(y_{1}+\frac{1}{y_{1}}+y_{2}+\frac{1}{y_{2}}+\frac{y_{2}}{y_{1}}+\frac{y_{1}}{y_{2}}\right) .
$$

Note that this kernel is a natural generalization of the previous case $f(y)=1+\frac{1}{2}(y+$ $\left.y^{-1}\right)$. We conjecture that the monopole formula of a quiver with $n \mathrm{U}(1)$ legs folds by integration over the kernel

$$
f\left(y_{1}, \ldots y_{n-1}\right)=1+\frac{1}{n} \chi_{f}^{A_{n-1}}\left(y_{1}, \ldots, y_{n-1}\right)
$$

where $\chi_{f}^{A_{n-1}}$ is the character of the $A_{n-1}$ fundamental representation.

The steps outlined above can be generalised to longer legs, larger gauge groups and, presumably, to completely arbitrary legs. However, rather than undertaking this task ourselves, we refer to [3] for a systematic look at the link between folding and the modified monopole formula of [1]. 


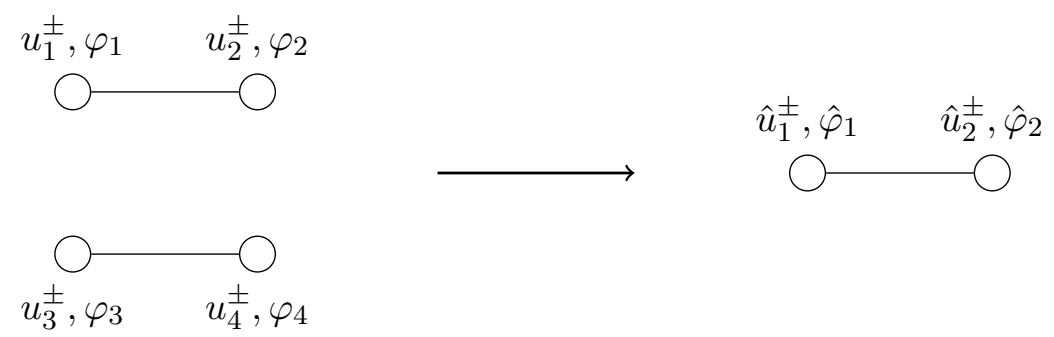

Figure 8. Example of folding two "parallel" links which do not originate from the same node. Note that folding does not introduce a multiple link in this case.

\subsection{Non-simply laced quivers}

It is possible to generalise abelianisation, including the Poisson structure, directly to non-simply laced framed quivers; the generalisation of the monopole formula was already achieved in [1]. The input data are a list of gauge nodes with optional fundamental matter and a connectivity matrix $\kappa$ defined precisely like the Cartan matrix of a Dynkin diagram. One can always unfold the quiver $\hat{Q}$ into a simply laced quiver $Q$. Keeping with the term's use in previous sections, the number of nodes of $Q$ which fold onto the $i$-th node of $\hat{Q}$ is called the multiplicity $\#_{i}$ of node $i$.

Each node still contributes three abelianised variables $\hat{u}_{i, a}^{ \pm}$and $\hat{\varphi}_{i, a}$ but the relations are slightly modified. They can be derived by demanding consistency with folding; recall that $x_{i, a}=\hat{x}_{i, a} / \#_{i}$ on the subspace preserved by discrete action. For simplicity we present them in the case of quivers with one multiple edge:

$$
\hat{u}_{i, a}^{+} \hat{u}_{i, a}^{-}=-\#_{i}^{2} \frac{\prod_{w \in \mathcal{R}}\langle w, \overrightarrow{\hat{\varphi}} / \overrightarrow{\#}\rangle^{g_{i}(w)\left|w_{i, a}\right|}}{\prod_{\alpha \in \Phi}\langle\alpha, \overrightarrow{\hat{\varphi}} / \overrightarrow{\#}\rangle^{\left|\alpha_{i, a}\right|}}
$$

where $\mathcal{R}$ is defined as if the quiver were simply laced (ie. the multiple link were replaced with one simple link), $\overrightarrow{\hat{\varphi}} / \overrightarrow{\#}$ denotes a vector of $\hat{\varphi}_{i, a} / \#_{i}$ and $g_{i}(w)$ is an auxiliary function defined as

$$
g_{i}(w)= \begin{cases}\left|\kappa_{j i}\right| & \text { if } w \text { connects the node } i \text { to node } j \\ 1 & \text { otherwise }\end{cases}
$$

and $\kappa$ is the Cartan matrix of the non-simply laced quiver.

The derivation of Poisson brackets is slightly more subtle. As a concrete example, consider a quiver with nodes 1 to 4 (plus possibly others) such that 1 and 2 , resp. 3 and 4 are connected, and 3 and 4 fold onto 1 and 2, respectively (see figure 8). Then

$$
\begin{aligned}
\left\{\hat{\varphi}_{1}, \hat{u}_{1}^{+}\right\} & =\left.\left\{\varphi_{1}+\varphi_{3}, u_{1}^{+}+u_{3}^{+}\right\}\right|_{x_{1}=x_{3}}=\left.\left(\left\{\varphi_{1}, u_{1}^{+}\right\}+\left\{\varphi_{3}, u_{3}^{+}\right\}\right)\right|_{x_{1}=x_{3}} \\
& =\varphi_{1}+\left.\varphi_{3}\right|_{\substack{x_{1}=x_{3} \\
x_{2}=x_{4}}}=\hat{\varphi}_{1}
\end{aligned}
$$

Similarly, and keeping to the same quiver for this example,

$$
\left\{\hat{u}_{1}^{+}, \hat{u}_{2}^{+}\right\}=\left.\left\{u_{1}^{+}+u_{3}^{+}, u_{2}^{+}+u_{4}^{+}\right\}\right|_{\substack{x_{1}=x_{3} \\ x_{2}=x_{4}}}=2\left\{u_{1}^{+}, u_{2}^{+}\right\}=2 \kappa_{12} \frac{u_{1}^{+} u_{2}^{+}}{\varphi_{1}-\varphi_{2}}=2 \kappa_{12} \frac{\hat{u}_{1}^{+} / 2 \hat{u}_{2}^{+} / 2}{\hat{\varphi}_{1} / 2-\hat{\varphi}_{2} / 2}
$$

Note that the factor of 2 comes from the two links which fold onto each other. 
Now that the procedure is clear it readily generalises:

$$
\begin{aligned}
& \left\{\hat{\varphi}_{i, a}, \hat{u}_{i, a}^{ \pm}\right\}= \pm \hat{u}_{i, a}^{ \pm} \\
& \left\{\hat{u}_{i, a}^{+}, \hat{u}_{i, a}^{-}\right\}=\#_{i}^{2} \frac{\partial}{\partial \hat{\varphi}_{i, a}} \frac{\prod_{w \in \mathcal{R}}\langle w, \overrightarrow{\hat{\varphi}} / \overrightarrow{\#}\rangle^{g_{i}(w)\left|w_{i, a}\right|}}{\prod_{\alpha \in \Phi}\langle\alpha, \vec{\varphi} / \overrightarrow{\#}\rangle^{\left|\alpha_{i, a}\right|}} \\
& \left\{\hat{u}_{i, a}^{ \pm}, \hat{u}_{j, b}^{ \pm}\right\}= \pm \kappa_{i j}^{S} \frac{\#_{i j}}{\#_{i} \#_{j}} \frac{\hat{u}_{i, a}^{ \pm} \hat{u}_{j, b}^{ \pm}}{\hat{\varphi}_{i, a} / \#_{i}-\hat{\varphi}_{j, b} / \#_{j}}
\end{aligned}
$$

where $\kappa^{S}$ is a "simply laced" Cartan matrix defined as $\kappa_{i j}^{S}=\max \left(\kappa_{i j}, \kappa_{j i}\right)$ (essentially throwing away information about multiplicity of edges) and $\#_{i j}$ is the link multiplicity of the edge between nodes $i$ and $j$ defined as the number of its pre-images in the unfolded quiver. Remember that just as in the case of abelianised relations this form is appropriate for quivers with one multiple edge.

\section{$5 \quad$ Examples}

In this section we study several cases of nilpotent orbit quivers, ie. quiver theories whose Coulomb branches are nilpotent orbits. Their chiral rings are generated by moment maps, which we explicitly construct; recall that such moment maps transform in the coadjoint representation $\operatorname{coadj}(\mathfrak{g}) \simeq \operatorname{adj}(\mathfrak{g})$ of the Coulomb branch symmetry. The chiral ring data is completed by providing a set of relations which also form representations of the Coulomb branch symmetry. We discretely gauge and fold such quivers and examine the resulting Coulomb branches in turn.

Most spaces encountered in this section are nilpotent orbits; their coordinate rings are generated by a single coadjoint representation. But there are a few cases which are not nilpotent orbits: their Coulomb branches are generated not only by $\operatorname{coadj}(\mathfrak{g})$ but also by chiral ring elements in other representations of $\mathfrak{g}$. If the quiver is balanced, for the examples studied in this paper, we find that the remaining generators also assemble into coadjoint (or sometimes trivial) representations and the bulk of our techniques still applies. One such case appears in section 5.2.5. The resulting spaces are not as comprehensively tabulated as nilpotent orbits and we generally have to turn to more varied sources to find their Hilbert series or highest weight generating functions.

\section{$5.1 \min A_{3} \rightarrow\left(\right.$ n.) $\min C_{2}$}

$A$-type quivers tend to have very simple moment maps which can be presented in reasonably compact form, allowing us to present the action of discrete gauging and folding.

The quivers we choose, as exhibited in table 9 , also exhibit an interesting pattern of complex mass deformation. As a general rule, all $\varphi_{i, a}$ abelian moduli and $M_{i, a}$ parameters only appear in the abelian algebra as differences and as a result the moduli space is invariant under reparametrisations $\varphi_{i, a} \rightarrow \varphi_{i, a}+c, M_{i, a} \rightarrow M_{i, a}+c$. Since there are precisely two mass parameters, the moduli space relations can be modified by terms proportional to $M_{1}-M_{3}$, ie. a complex mass deformation. However both discrete gauging and folding remove one half of mass parameters by forcing $M_{1}=M_{3}$, which can in turn be set to 0 by 


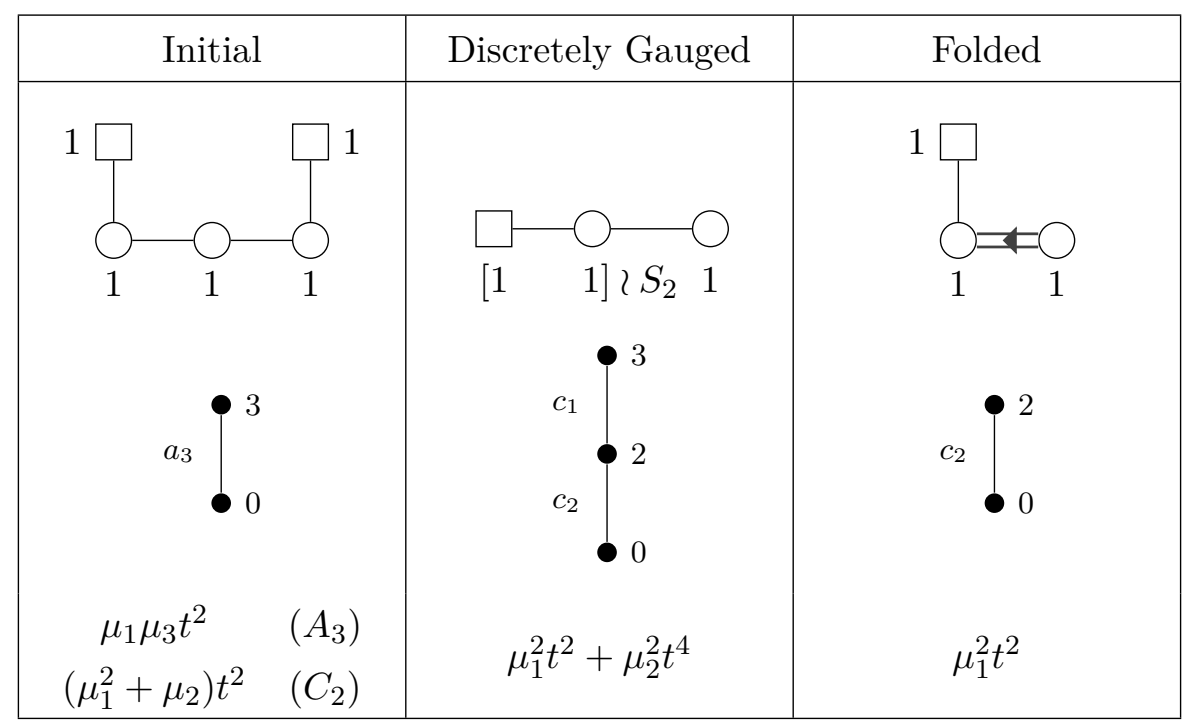

Table 9. $A_{3}$ minimal nilpotent orbit and its discrete reductions.

the reparametrisation above. As a result only the original space can be deformed by one triplet of mass parameters.

\subsubsection{Initial quiver}

To remind the reader we reproduce abelianised relations restricting $u_{i}^{ \pm}, \varphi_{i}$ for $i=1,2,3$ :

$$
\begin{aligned}
& u_{1}^{+} u_{1}^{-}=-\left(\varphi_{1}-M_{1}\right)\left(\varphi_{1}-\varphi_{2}\right) \\
& u_{2}^{+} u_{2}^{-}=-\left(\varphi_{2}-\varphi_{1}\right)\left(\varphi_{2}-\varphi_{3}\right) \\
& u_{3}^{+} u_{3}^{-}=-\left(\varphi_{3}-\varphi_{2}\right)\left(\varphi_{3}-M_{3}\right)
\end{aligned}
$$

The Coulomb branch is generated by

$$
N_{A_{3}}=\left(\begin{array}{cccc}
\varphi_{1}-\frac{3 M_{1}+M_{3}}{4} & u_{1}^{-} & -\frac{u_{1}^{-} u_{2}^{-}}{\varphi_{1}-\varphi_{2}} & \frac{u_{1}^{-} u_{2}^{-} u_{3}^{-}}{\left(\varphi_{1}-\varphi_{2}\right)\left(\varphi_{2}-\varphi_{3}\right)} \\
u_{1}^{+} & -\varphi_{1}+\varphi_{2}+\frac{M_{1}-M_{3}}{4} & u_{2}^{-} & -\frac{u_{2}^{-} u_{3}^{-}}{\left(\varphi_{2}-\varphi_{3}\right)} \\
-\frac{u_{1}^{+} u_{2}^{+}}{\varphi_{1}-\varphi_{2}} & u_{2}^{+} & -\varphi_{2}+\varphi_{3}+\frac{M_{1}-M_{3}}{4} & u_{3}^{-} \\
\frac{u_{1}^{+} u_{2}^{+} u_{3}^{+}}{\left(\varphi_{1}-\varphi_{2}\right)\left(\varphi_{2}-\varphi_{3}\right)} & -\frac{u_{2}^{+} u_{3}^{+}}{\left(\varphi_{2}-\varphi_{3}\right)} & u_{3}^{+} & -\varphi_{3}+\frac{M_{1}+3 M_{3}}{4}
\end{array}\right)
$$

and one can read its relations either from the HWG [30]

$$
\operatorname{HWG}\left(t, \mu_{i}\right)=\frac{1}{1-\mu_{1} \mu_{3} t^{2}}
$$

or simply from the Joseph relations, which are obeyed by any minimal nilpotent orbit:

$$
\begin{aligned}
& t^{4}([101]+[000]): \quad N^{2}=-\frac{1}{2}\left(M_{1}-M_{3}\right) N+\frac{3}{16}\left(M_{1}-M_{3}\right)^{2} \mathbf{1} \\
& t^{4}[020]: \quad \sum_{a^{\prime}, b^{\prime}} \varepsilon_{a^{\prime} b^{\prime}[c d} N_{a}^{a^{\prime}} N_{b]}^{b^{\prime}}=-\frac{1}{16}\left(M_{1}-M_{3}\right)^{2} \varepsilon_{a b c d}
\end{aligned}
$$




\subsubsection{Discrete gauging}

The $A_{3}$ moment map discretely gauges to the following expression:

$$
N_{C_{2}}=\left(\begin{array}{cccc}
\frac{1}{2}\left(\tilde{\varphi}_{1}+\tilde{\varphi}_{3}\right) & \frac{1}{2}\left(\tilde{u}_{1}^{-}+\tilde{u}_{3}^{-}\right) & \frac{1}{2}\left(-\frac{\tilde{u}_{1}^{-} \tilde{u}_{2}^{-}}{\tilde{\varphi}_{1}-\tilde{\varphi}_{2}}+\frac{\tilde{u}_{2}^{-} \tilde{u}_{3}^{-}}{\tilde{\varphi}_{2}-\tilde{\varphi}_{3}}\right) & \frac{\tilde{u}_{1}^{-} \tilde{u}_{2}^{-} \tilde{u}_{3}^{-}}{\left(\tilde{\varphi}_{1}-\tilde{\varphi}_{2}\right)\left(\tilde{\varphi}_{2}-\tilde{\varphi}_{3}\right)} \\
\frac{1}{2}\left(\tilde{u}_{1}^{+}+\tilde{u}_{2}^{+}\right) & -\frac{1}{2}\left(\tilde{\varphi}_{1}+\tilde{\varphi}_{3}\right)+\tilde{\varphi}_{2} & \tilde{u}_{2}^{-} & \frac{1}{2}\left(\frac{\tilde{u}_{1}^{-} \tilde{u}_{2}^{-}}{\tilde{\varphi}_{1}-\tilde{\varphi}_{2}}-\frac{\tilde{u}_{2}}{\tilde{\varphi}_{2}-\tilde{u}_{3}}\right) \\
\frac{\tilde{u}_{1}^{+} \tilde{u}_{2}^{+}}{2}\left(-\frac{\tilde{u}_{2}^{+} \tilde{u}_{3}^{+}}{\tilde{\varphi}_{1}-\tilde{\varphi}_{2}}\right) & \tilde{u}_{2}^{+} & -\tilde{\varphi}_{2}+\frac{1}{2}\left(\tilde{\varphi}_{1}+\tilde{\varphi}_{3}\right) & \frac{1}{2}\left(\tilde{u}_{1}^{-}+\tilde{u}_{3}^{-}\right) \\
\frac{\tilde{u}_{1}^{+} \tilde{u}_{2}^{+} \tilde{u}_{3}^{+}}{\left(\tilde{\varphi}_{1}-\tilde{\varphi}_{2}\right)\left(\tilde{\varphi}_{2}-\tilde{\varphi}_{3}\right)} & \frac{1}{2}\left(\frac{\tilde{u}_{1}^{+} \tilde{u}_{2}^{+}}{\tilde{\varphi}_{1}-\tilde{\varphi}_{2}}-\frac{\tilde{u}_{2}^{+} \tilde{u}_{3}^{+}}{\tilde{\varphi}_{2}-\tilde{\varphi}_{3}}\right) & \frac{1}{2}\left(\tilde{u}_{1}^{+}+\tilde{u}_{2}^{+}\right) & \frac{1}{2}\left(-\tilde{\varphi}_{1}-\tilde{\varphi}_{3}\right)
\end{array}\right)
$$

and the resulting space is expected to have quaternionic dimension 3 and exhibit $C_{2}$ symmetry. The next-to-minimal nilpotent orbit of $C_{2}$ is a suitable candidate. Its HWG reads [30]

$$
\operatorname{HWG}\left(t, \mu_{i}\right)=\frac{1}{\left(1-\mu_{1}^{2} t^{2}\right)\left(1-\mu_{2}^{2} t^{4}\right)}
$$

suggesting several relations:

$$
\begin{aligned}
t^{4}([00]+[01]): & N^{2}=0 \\
t^{6}[20]: & \operatorname{rank}(N)
\end{aligned}
$$

(Note that in our convention we multiply $C_{n}$ matrices as ordinary matrices, ie. without insertion of an $\epsilon$ tensor.) The second of these relations can be written equivalently as

$$
\sum_{b, c, d, b^{\prime}, c^{\prime}, d^{\prime}} \varepsilon_{a^{\prime} b^{\prime} c^{\prime} d^{\prime}} \varepsilon^{a b c d} N_{b}^{b^{\prime}} N_{c}^{c^{\prime}} N_{d}^{d^{\prime}}=0
$$

In other words, an explicit algebraic description of the Coulomb branch of the discretely gauged quiver is

$$
\left\{N \in \mathfrak{g l}(4, \mathbb{C}) \mid N^{2}=0, \quad \operatorname{rank}(N) \leq 2, \quad N^{T} J-J N=0\right\} .
$$

\subsubsection{Folding}

The folded moment map is similar:

$$
N_{C_{2}}=\left(\begin{array}{cccc}
\frac{1}{2} \hat{\varphi}_{1} & \frac{1}{2} \hat{u}_{1}^{-} & -\frac{\hat{u}_{1}^{-} \hat{u}_{2}^{-}}{\hat{\varphi}_{1}-2 \hat{\varphi}_{2}} & -\frac{\left(\hat{u}_{1}^{-}\right)^{2} \hat{u}_{2}^{-}}{\left(\hat{\varphi}_{1}-2 \hat{\varphi}_{2}\right)^{2}} \\
\frac{1}{2} \hat{u}_{1}^{+} & -\frac{1}{2} \hat{\varphi}_{1}+\hat{\varphi}_{2} & \hat{u}_{2}^{-} & \frac{\hat{u}_{2}^{-} \hat{u}_{1}^{-}}{\left(\hat{\varphi}_{1}-2 \hat{\varphi}_{2}\right)} \\
-\frac{\hat{u}_{1}^{+} \hat{u}_{2}^{+}}{\hat{\varphi}_{1}-2 \hat{\varphi}_{2}} & \hat{u}_{2}^{+} & -\hat{\varphi}_{2}+\frac{1}{2} \hat{\varphi}_{1} & \frac{1}{2} \hat{u}_{1}^{-} \\
-\frac{\left(\hat{u}_{1}^{+}\right)^{2} \hat{u}_{2}^{+}}{\left(\hat{\varphi}_{1}-2 \hat{\varphi}_{2}\right)^{2}} & \frac{\hat{u}_{2}^{+} \hat{u}_{1}^{+}}{\left(\hat{\varphi}_{1}-2 \hat{\varphi}_{2}\right)} & \frac{1}{2} \hat{u}_{1}^{+} & -\frac{1}{2} \hat{\varphi}_{1}
\end{array}\right)
$$

The Coulomb branch has dimension 2 and $C_{2}$ symmetry, which agrees with the minimal nilpotent orbit with HWG [30]

$$
\operatorname{HWG}\left(t, \mu_{i}\right)=\frac{1}{1-\mu_{1}^{2} t^{2}}
$$

This space satisfies slightly more stringent (Joseph) relations:

$$
\begin{aligned}
t^{4}([00]+[01]): & N^{2}=0 \\
t^{4}[02]: & \operatorname{rank}(N) \leq 1
\end{aligned}
$$


The second of these relations can be written equivalently as $N_{[a}^{a^{\prime}} N_{b]}^{b^{\prime}}=0$. In other words, an explicit algebraic description of the Coulomb branch of the folded quiver is

$$
\left\{N \in \mathfrak{g l}(4, \mathbb{C}) \mid N^{2}=0, \quad \operatorname{rank}(N) \leq 1, \quad N^{T} J-J N=0\right\} .
$$

\section{$5.2 \min D_{4} \rightarrow G_{2}$}

$G_{2}$ is small yet non-trivial enough to serve as an excellent illustration of the techniques studied in this paper. Since it is only fourteen-dimensional, we provide the complete folding prescription from both $D_{4}$ and $B_{3}$ :

$$
\begin{aligned}
G_{2} & =\operatorname{span}_{\mathbb{C}}\left(E_{ \pm 1^{3} 2^{2}}^{G_{2}}, E_{ \pm 1^{3} 2}^{G_{2}}, E_{ \pm 1^{2} 2}^{G_{2}}, E_{ \pm 12}^{G_{2}}, E_{ \pm 1}^{G_{2}}, E_{ \pm 2}^{G_{2}}, H_{1}^{G_{2}}, H_{2}^{G_{2}}\right) \\
E_{ \pm 1^{3} 2^{2}}^{G_{2}} & =E_{ \pm 12^{2} 34}^{D_{4}}=E_{ \pm 12^{2} 3^{2}}^{B_{3}} \\
E_{ \pm 1^{3} 2}^{G_{2}} & =E_{ \pm 1234}^{D_{4}}=E_{ \pm 123^{2}}^{B_{3}} \\
E_{ \pm 1^{2} 2}^{G_{2}} & =-E_{ \pm 123}^{D_{4}}-E_{ \pm 124}^{D_{4}}+E_{ \pm 234}^{D_{4}}=-E_{ \pm 123}^{B_{3}}+E_{ \pm 23^{2}}^{B_{3}} \\
E_{ \pm 12}^{G_{2}} & =E_{ \pm 12}^{D_{4}}-E_{ \pm 23}^{D_{4}}-E_{ \pm 24}^{D_{4}}=E_{ \pm 12}^{B_{3}}-E_{ \pm 23}^{B_{3}} \\
E_{ \pm 1}^{G_{2}} & =E_{ \pm 1}^{D_{4}}+E_{ \pm 3}^{D_{4}}+E_{ \pm 4}^{D_{4}}=E_{ \pm 1}^{B_{3}}+E_{ \pm 3}^{B_{3}} \\
E_{ \pm 2}^{G_{2}} & =E_{ \pm 2}^{D_{4}}=E_{ \pm 2}^{B_{3}} \\
H_{ \pm 1}^{G_{2}} & =H_{ \pm 1}^{D_{4}}+H_{ \pm 3}^{D_{4}}+H_{ \pm 4}^{D_{4}}=H_{ \pm 1}^{B_{3}}+H_{ \pm 3}^{B_{3}} \\
H_{ \pm 2}^{G_{2}} & =H_{ \pm 2}^{D_{4}}=H_{ \pm 2}^{B_{3}}
\end{aligned}
$$

Recall that $G_{2}$ is characterised as the subalgebra of $B_{3}$ which preserves a particular rank 3 antisymmetric tensor $\phi$; for more details see section 2.1 .

The goal of this subsection is to identify quivers whose Coulomb branches are generated by operators in one $G_{2}$ coadjoint representation [01]; such spaces are necessarily nilpotent orbits. We also study one related space whose coordinate ring is generated by coadjoint generators but is not a nilpotent orbit. The following sections should be read alongside tables 10 and 11 .

Note that because the quiver has only flavor node of rank 1 , the $G_{2}$ spaces studied below cannot be deformed by a complex mass.

We provide the first few symmetric products of the (co)adjoint representation for reference:

$$
\begin{aligned}
& \operatorname{Sym}^{2}[01]=[20]+[00]+[02] \\
& \operatorname{Sym}^{3}[01]=[30]+[21]+[01]+[10]+[03] \\
& \operatorname{Sym}^{4}[01]=[40]+[31]+[22]+[11]+2[20]+[00]+2[02]+[04]
\end{aligned}
$$




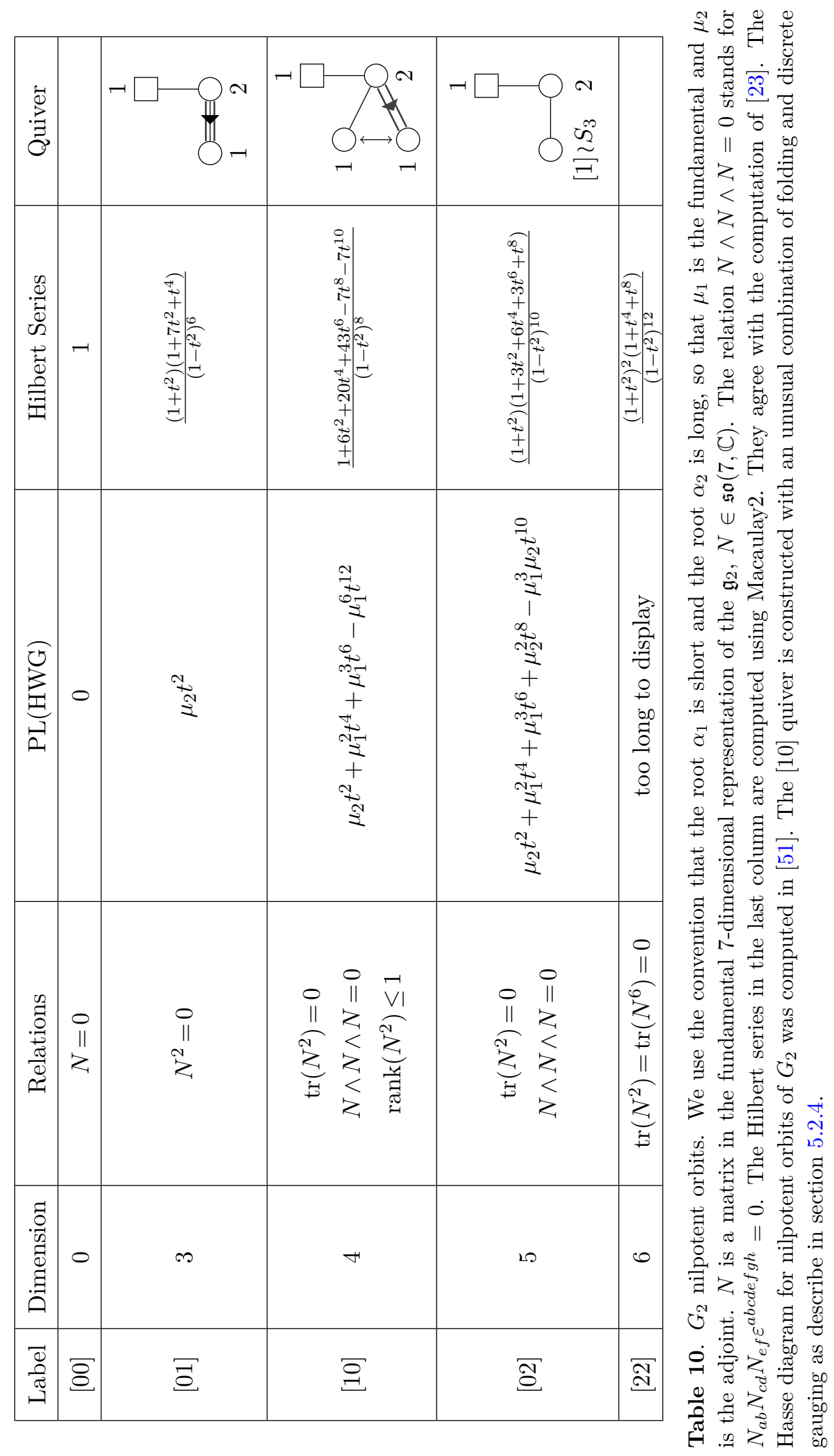




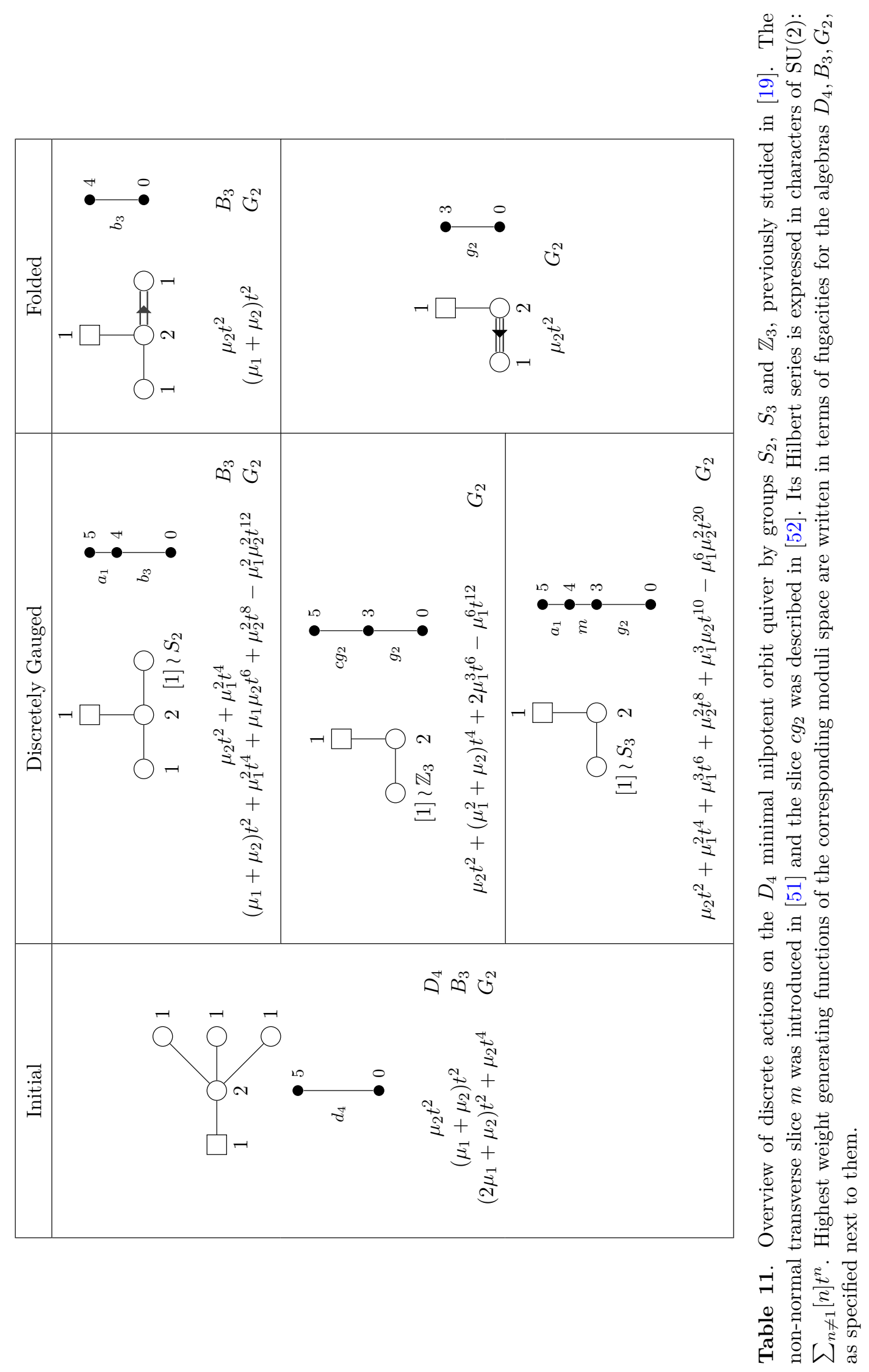




\subsubsection{Initial quiver}

The next few examples share the quiver on the left of table 11 as the common starting point. Its Coulomb branch is the minimal nilpotent orbit of $D_{4}$ which is parametrised by a coadjoint (antisymmetric) matrix $M$ subject to the Joseph relations

$$
\begin{array}{lr}
([2000]+[0000]) t^{4}: & N^{2}=0 \\
([0020]+[0002]) t^{4}: & N \wedge N=0
\end{array}
$$

We refer the reader to its treatment in [7] for more details.

\subsubsection{Folding}

The minimal nilpotent orbit of $D_{4}$ folds into the minimal nilpotent orbit of $G_{2}$ whose quiver is depicted in table 10 under the label [01]. To verify this claim we can look at the highest weight generating function of the minimal nilpotent orbit of $G_{2}$ [30]

$$
\operatorname{HWG}(t)=\frac{1}{1-\mu_{2} t^{2}}=1+\mu_{2} t^{2}+\mu_{2}^{2} t^{4}+\ldots
$$

or recall that the Joseph relations tell us that the coadjoint generator is constrained by the quadratic relation

$$
([20]+[00]) t^{4}: N^{2}=0 .
$$

Direct computation shows that the relation is satisfied by $N$ defined either by folding the moment map of the $D_{4}$ minimal nilpotent orbit quiver or directly using the non-simply laced prescription.

\subsection{3 $\quad S_{3}$ discrete gauging}

The five-dimensional subregular orbit of $G_{2}$ is known to be the $S_{3}$ quotient of the minimal nilpotent orbit of $D_{4}[5]$ so it should be the Coulomb branch of the appropriate $D_{4}$ quiver after discrete gauging, see row [02] of table 10. One can either symmetrise the $D_{4}$ moment map using the projector defined in (3.12) or, given the $G_{2}$ Chevalley Serre basis $\left\{X_{i}\right\}$, form the $G_{2}$ moment map $N_{G_{2}}$ from its $D_{4}$ counterpart $N_{D_{4}}$ as

$$
N_{G_{2}}=\sum_{i} X_{i}^{*} \operatorname{tr}\left(N_{D_{4}} X_{i}\right)
$$

The highest weight generating function is

$$
\begin{aligned}
& \operatorname{HWG}(t)=\frac{1+\mu_{1}^{3} \mu_{2} t^{10}}{\left(1-\mu_{2} t^{2}\right)\left(1-\mu_{1}^{2} t^{4}\right)\left(1-\mu_{1}^{3} t^{6}\right)\left(1-\mu_{2}^{2} t^{8}\right)} \\
& =1+\mu_{2} t^{2}+\left(\mu_{1}^{2}+\mu_{2}^{2}\right) t^{4}+\left(\mu_{1}^{3}+\mu_{2} \mu_{1}^{2}+\mu_{2}^{3}\right) t^{6}+\left(\mu_{1}^{4}+\mu_{2} \mu_{1}^{3}+\mu_{2}^{2} \mu_{1}^{2}+\mu_{2}^{4}+\mu_{2}^{2}\right) t^{8}+\ldots
\end{aligned}
$$

Two relations are needed this time:

$$
\begin{array}{rr}
{[00] t^{4}:} & \operatorname{tr} N^{2}=0 \\
{[10] t^{6}:} & N \wedge N \wedge N=0
\end{array}
$$

and both are satisfied by the coadjoint $N_{G_{2}}$. 


\subsubsection{Mixed folding and $S_{2}$ gauging}

Midway between the two previous examples lies a nilpotent orbit of dimension 4. It is known [23] to be non-normal and hence not expected to be the Coulomb branch of any quiver since both simply and non-simply laced quivers are necessarily normal [3, 18]. However we conjecture that it can be recovered by using a specific and non-generic discrete operation on the minimal nilpotent orbit quiver of $B_{3}$, which is itself four-dimensional. This would make our construction the first non-normal Coulomb branch in the literature.

We first construct the moment map $N_{B_{3}}$ of the underlying $B_{3}$ quiver. The quiver has no obvious automorphism so rather than using the projector form in (3.12) we define the Chevalley-Serre basis $\left\{X_{i}\right\}$ of $G_{2}$ and project using the trick from the previous quiver calculation:

$$
N_{G_{2}}=\sum_{i} X_{i}^{*} \operatorname{tr}\left(N_{B_{3}} X_{i}\right)
$$

We depict the conjectured quiver theory in table 10 on row 10 .

The HWG of this orbit is given by $[30]^{17}$

$$
\begin{aligned}
& \operatorname{HWG}(t)=\frac{1-\mu_{1}^{6} t^{12}}{\left(1-\mu_{2} t^{2}\right)\left(1-\mu_{1}^{2} t^{4}\right)\left(1-\mu_{1}^{3} t^{6}\right)} \\
& =1+\mu_{2} t^{2}+\left(\mu_{1}^{2}+\mu_{2}^{2}\right) t^{4}+\left(\mu_{1}^{3}+\mu_{1}^{2} \mu_{2}+\mu_{2}^{3}\right) t^{6}+\left(\mu_{1}^{4}+\mu_{1}^{3} \mu_{2}+\mu_{1}^{2} \mu_{2}^{2}+\mu_{2}^{4}\right) t^{8}+\ldots
\end{aligned}
$$

Compared to the subregular nilpotent orbit we find an extra relation at $t^{8}$ in the [02] representation. The condition that $N^{2}$ is of rank at most 1 is of this type.

In total the moment map is expected to satisfy three relations:

$$
\begin{array}{lr}
{[00] t^{4}:} & \operatorname{tr}\left(N^{2}\right)=0 \\
{[10] t^{6}:} & N \wedge N \wedge N=0 \\
{[02] t^{8}:} & \operatorname{rank}\left(N^{2}\right) \leq 1
\end{array}
$$

and indeed all are met by our coadjoint $N_{G_{2}}$. The last relation (5.34) can be written as $\sum_{m, n}\left(N_{a m} N_{m b} N_{c n} N_{n d}-N_{a m} N_{m d} N_{c n} N_{n b}\right)=0$. We have checked analytically that the three relations above form a complete set of relations.

\footnotetext{
${ }^{17}$ We can compare this expression with the HWG for the minimal $B_{3}$ orbit, written in terms of $G_{2}$ fugacities, which reads [30]

$$
\begin{aligned}
\operatorname{HWG}(t)=\frac{1}{\left(1-\mu_{1} t^{2}\right)\left(1-\mu_{2} t^{2}\right)}= & 1+\left(\mu_{1}+\mu_{2}\right) t^{2}+\left(\mu_{1}^{2}+\mu_{1} \mu_{2}+\mu_{2}^{2}\right) t^{4} \\
& +\left(\mu_{1}^{3}+\mu_{1}^{2} \mu_{2}+\mu_{1} \mu_{2}^{2}+\mu_{2}^{3}\right) t^{6}+\left(\mu_{1}^{4}+\mu_{1}^{3} \mu_{2}+\mu_{1}^{2} \mu_{2}^{2}+\mu_{1} \mu_{2}^{3}+\mu_{2}^{4}\right) t^{8}+\ldots
\end{aligned}
$$

The difference between the two expressions is

$$
\frac{\mu_{1} t^{2}}{1-\mu_{2} t^{2}}=\mu_{1} t^{2}+\mu_{1} \mu_{2} t^{4}+\mu_{1} \mu_{2}^{2} t^{6}+\mu_{1} \mu_{2}^{3} t^{8}+\ldots
$$




\subsection{5 $\mathbb{Z}_{3}$ discrete gauging}

Although elsewhere in the paper we discretely gauge or fold $S_{m}$ quiver automorphisms, discrete gauging by a subset of $S_{m}$ is perfectly well defined. Here we consider the $\mathbb{Z}_{3}$ discrete gauging of the $D_{4}$ quiver studied in this section. Its Coulomb branch was previously investigated in [19] under the name $\mathcal{C}_{D_{4}^{(3)}}$. The plethystic logarithm of its highest weight generating function was reported as ${ }^{18}$

$$
P L(t)=[01] t^{2}+([01]-[00]) t^{4}-([01]+[10]+[20]+[00]) t^{6}-([01]+[10]-[02]) t^{8}+O\left(t^{10}\right) .
$$

This space is not a nilpotent orbit. It is generated by two coadjoint matrices at quadratic and quartic order in $t$ respectively. The lower coadjoint matrix $N$ is also the moment map and looks precisely like the one obtained by $S_{3}$ symmetric gauging. Since $\mathbb{Z}_{3} \subset S_{3}$, there are operators in this theory which are removed if the remaining $S_{2} \subset S_{3}$ symmetry is imposed. One of the simplest operators is

$$
\tilde{e}_{\langle 10\rangle}^{4}=u_{1}^{+}\left(\varphi_{4}-\varphi_{3}\right)+u_{3}^{+}\left(\varphi_{1}-\varphi_{4}\right)+u_{4}^{+}\left(\varphi_{3}-\varphi_{1}\right) .
$$

As its label suggests, $\tilde{e}_{\langle 10\rangle}^{4}$ is a $t^{4}$ operator which acts as the first simple root under action of the moment map's components. And just as one can "rotate" a simple root into any other root by repeated action of the Lie bracket, it is possible to repeatedly act with the Poisson bracket on $\tilde{e}_{\langle 10\rangle}^{4}$ to generate an entire $t^{4}$ adjoint representation's worth of operators which can be bundled together to form the second coadjoint matrix $R$. For example:

$$
\tilde{e}_{\langle 01\rangle}^{4}=-\left\{\tilde{e}_{\langle-10\rangle},\left\{\tilde{e}_{\langle 01\rangle}, \tilde{e}_{\langle 10\rangle}^{4}\right\}\right\}
$$

The plethystic logarithm suggests several relations between $N$ and $R$ but we find it is not too helpful in this case. For example, its syzygies obscure several relations at order $t^{8}$. Accordingly, we opt for a different approach to identify the relations. Ref. [19] identifies a non-simply laced quiver with the same Coulomb branch, which is itself a folded version of the quiver in figure 8 of [31]; the latter paper reports matrix relations. In general folded relations follow the form of the original quiver's; indeed they must as they are merely the original relations restricted to the folded subspace. Accounting for several coincidences in $G_{2}\left(\right.$ eg $\left.N^{3} \propto\left(\operatorname{tr} N^{2}\right) N,\{N, R\} \propto N \wedge R\right)$ and a different numerical factor in the last relation, we are left with the following relations:

$$
\begin{aligned}
{[00] t^{4}: } & \operatorname{tr} N^{2} & =0 \\
{[10] t^{6}: } & N \wedge N \wedge N & =0 \\
{[01] t^{6}: } & {[N, R] } & =0 \\
([20]+[00]) t^{6}: & \{N, R\} & =0 \\
([20]+[00]) t^{8}: & R^{2} & =0 \\
{[02] t^{8}: } & \left(N^{2}\right)_{[a}^{[b}\left(N^{2}\right)_{c]}^{d]} & =\frac{1}{54} R_{a}^{b} R_{c}{ }^{d}
\end{aligned}
$$

We are able to verify all of them symbolically, but cannot guarantee that they form a minimal set of relations as our current techniques run against a computational limit.

\footnotetext{
${ }^{18}$ Paper [19] also follows the opposite root convention to the present paper.
} 


\section{$5.3 \quad D_{5} \rightarrow B_{4}$}

We close off by studying discrete gauging and folding on a family of quivers. Tables 12,13 and 14 present results of discrete gauging and folding on three $D_{5}$ nilpotent orbit quivers. The Hilbert series, HWGs and quivers were originally reported in [30, 53].

Tables 12-14 follow the same pattern. The first line shows the unitary magnetic quivers. The second line shows the equivalent orthosymplectic magnetic quivers (ie. with the same Coulomb branch); our discrete gauging appears to be the unitary analogue of gauging an $O(1)$ group in these quivers as studied in [53]. The third line shows an electric quiver, by which we mean a classical quiver theory whose Higgs branch is the Coulomb branch under study. Several quivers may share this property; in particular the ones chosen here need not be the $3 d$ mirrors. Note in those electric quivers the appearance of an $O_{1}=\mathbb{Z}_{2}$ gauge group in the middle column. The last lines show the Hasse diagrams, HWG and relations. The HWG use $B_{4}$ fugacities except in the first column where $D_{5}$ fugacities are also used.

We draw the reader's attention to several interesting properties.

Firstly, a $D$-type moment map in the Chevalley-Serre basis is too long to print but both discrete gauging and folding have clear and discernible effects on it. The original, unfolded moment map transforms in the coadjoint (antisymmetric) matrix representation of $\mathfrak{s o}(10, \mathbb{C})$. Upon either discrete operation, all components along the last row and column vanish and the originally $10 \times 10$ matrix effectively becomes a $9 \times 9$ antisymmetric matrix padded by zeroes - and hence transforms in the coadjoint representation of $\mathfrak{s o}(9, \mathbb{C})$.

Secondly, in the case of the next-to-next-to-minimal nilpotent orbit we wreathe a $\mathrm{U}(2)$ node rather than the simple and well understood case of $\mathrm{U}(1)$, demonstrating that discrete gauging generalises to gauge ranks higher than 1. Finally, in the same example, each wreathed $\mathrm{U}(2)$ node comes with one flavor so the triplet of spaces exhibits interesting complex mass deformation behaviour analogous to that of section 5.1: only the initial space can be deformed by complex mass, and turning on two inequivalent mass parameters spoils the $S_{2}$ symmetry required for both discrete gauging and folding.

Note that notation of the form $N \wedge \cdots \wedge N$ denotes antisymmetrisation over all indices, or equivalently contraction with the appropriate Levi-Civita tensor.

In tables 12-14, we have colored the terms of the HWG which are charged under the $\mathbb{Z}_{2}$ action in violet. 


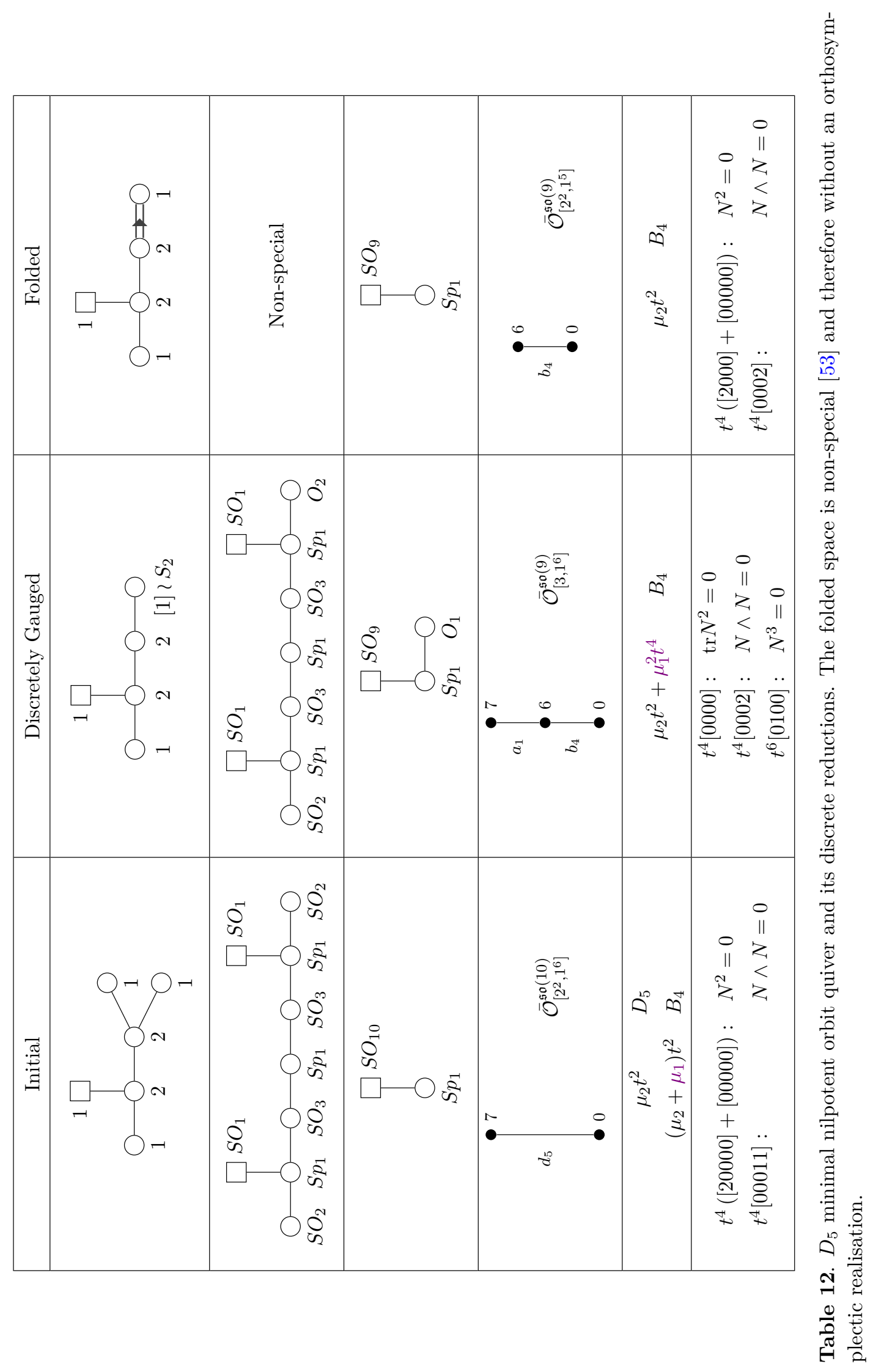




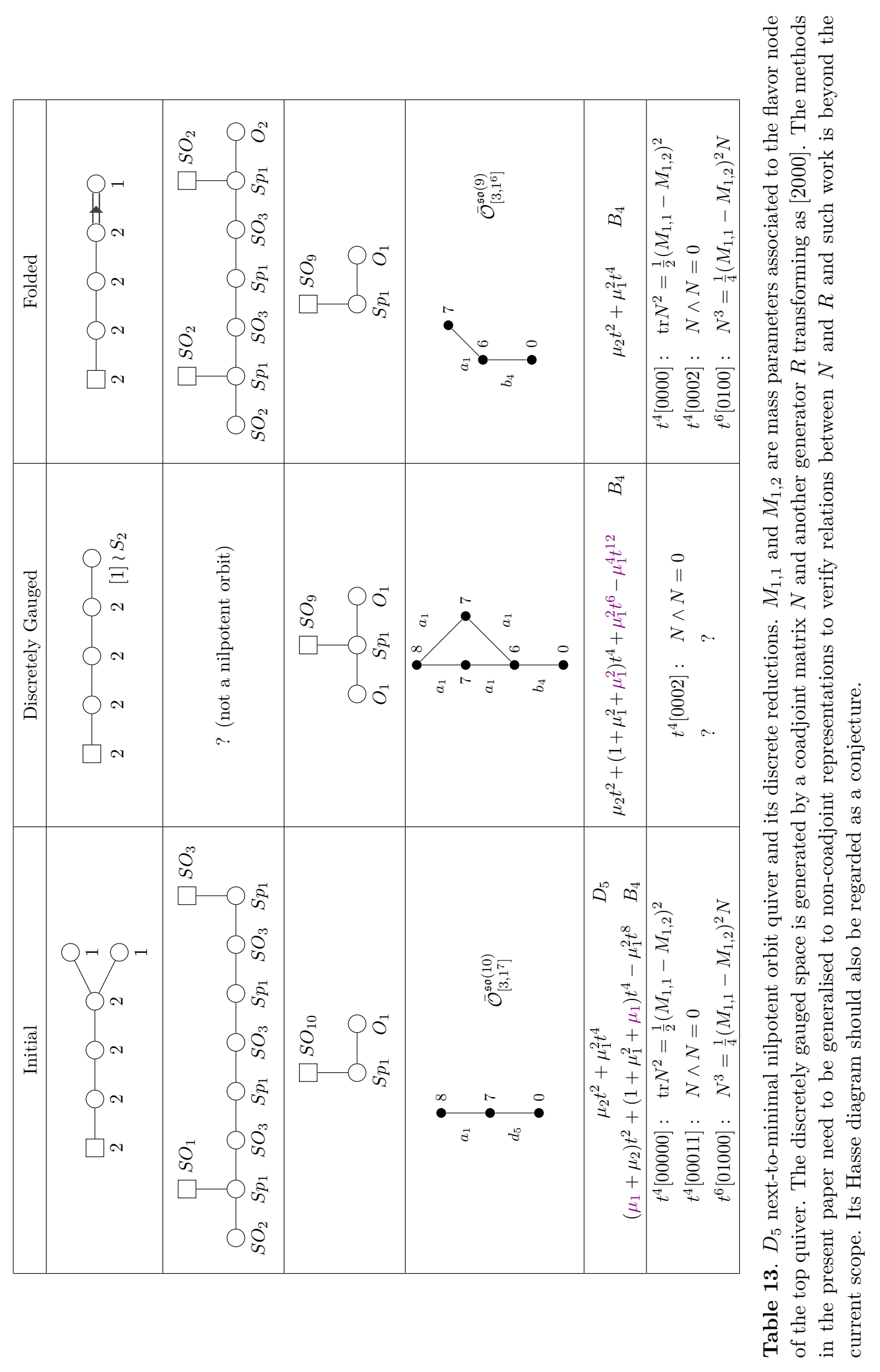




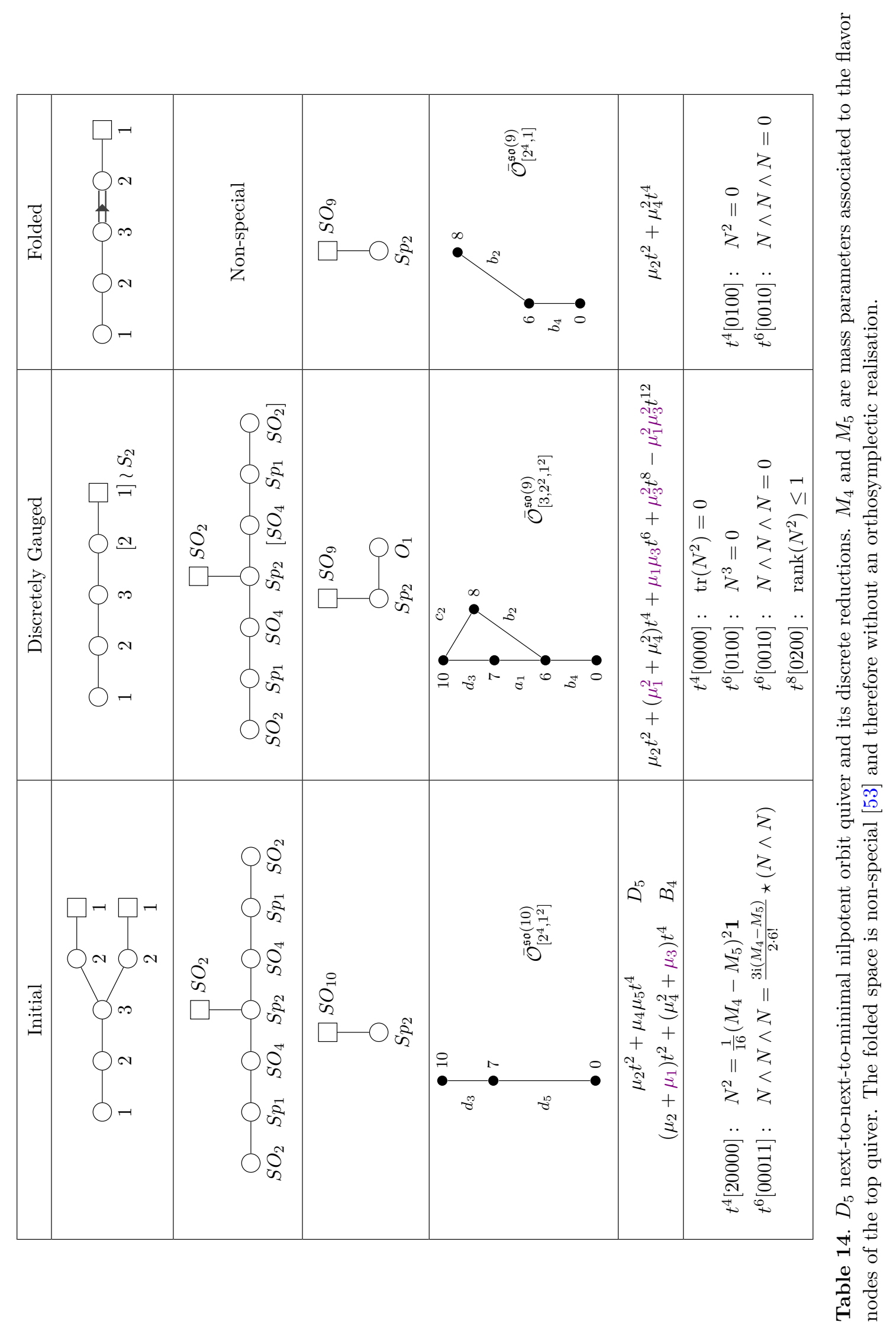




\section{Acknowledgments}

The authors are grateful to Julius Grimminger and Zhenghao Zhong for clearing up an important point about discrete gauging and useful comments on the draft and to an anonymous reviewer for insightful feedback. D.M. is supported by STFC DTP research studentship grant ST/N504336/1 while A.B. and A.H. are supported by STFC grant $\mathrm{ST} / \mathrm{P} 000762 / 1$. We are grateful to organisers of the 11th Joburg Workshop on String Theory for their hospitality, and the MIT-Imperial College London Seed Fund for support.

\section{A U(2) with 4 flavors and abelianised rings}

The main text describes a two-stage method to explicitly construct the chiral ring: first generate a set of abelianised operators and then, guided by representation-theoretic data from the Hilbert series, select certain Weyl-invariant combinations to form tensor generators of the ring and verify that they satisfy the correct relations. The method does not require a deep dive into abelianisation and the precise relation between $\mathbb{C}\left[\mathcal{C}_{\text {abel }}\right]$ and $\mathbb{C}[\mathcal{C}]$, so we leave such discussion out of the main body of this paper and address some of the potential concerns in this appendix.

First, we set the stage. Starting with abelianised variables, the Poisson bracket (2.35) generates new elements of the abelianised ring which cannot be expressed by adding and multiplying basic variables. The full abelianised ring ${ }^{19} \mathbb{C}\left[\mathcal{C}_{\text {abel }}\right]$ is constructed as the ring underlying the Poisson algebra generated by abelianised variables $u_{i, a}^{ \pm}$and $\varphi_{i, a}$ subject to relations (2.30) and (2.33)-(2.35).

In the interest of concreteness consider the case of a $U(2)$ theory with 4 flavors. Elements such as $1 /\left(\varphi_{1}-\varphi_{2}\right)$ (inverse masses of $W$-bosons) never appear in $\mathbb{C}\left[\mathcal{C}_{\text {abel }}\right]$ on their own. On the other hand, the abelianised relation

$$
u_{1}^{+} u_{1}^{-}=-\frac{\prod_{1 \leq i \leq 4}\left(\varphi_{1}-M_{i}\right)}{\left(\varphi_{1}-\varphi_{2}\right)^{2}}
$$

\footnotetext{
${ }^{19}$ We stress that this notion of the abelianised ring is a departure from that of [4], where it is introduced in (4.9) (with minor notational differences) as

$$
\mathbb{C}\left[\mathcal{M}_{\mathcal{C}}^{\text {abel }}\right]=\left(\mathbb{C}\left[\left\{u_{A}^{ \pm}\right\},\left\{\varphi_{a}\right\},\left\{\left(M_{j}^{W}\right)^{-1}\right\}_{j \in \text { roots }}\right] /(\text { abelianised relations })\right)^{\mathcal{W}_{G}} .
$$

In essence, this "abelianised" ring is the Weyl-invariant part of a ring generated by all abelianised monopoles, scalar operators and all inverse masses (i.e. $\left.1 /\left(\varphi_{a}-\varphi_{b}\right)\right)$ modulo relations between abelianised monopoles. The authors follow with clarification that this is emphatically not the Coulomb branch chiral ring, since a) the inverse masses are not defined everywhere on it (i.e. when $\varphi_{a}=\varphi_{b}$ ), and even if one restricts to the "discriminant locus" of the Coulomb branch (that is, the points where $\varphi_{a} \neq \varphi_{b}$ ), b) this ring contains extra elements.

To see that the two "abelianised" rings $\mathbb{C}\left[\mathcal{M}_{\mathcal{C}}^{\text {abel }}\right]$ and $\mathbb{C}\left[\mathcal{C}_{\text {abel }}\right]$ are inequivalent, take the theory $\mathrm{U}(2)$ with 4 flavors (as in the remainder of this appendix). Then the element $1 /\left(\varphi_{1}-\varphi_{2}\right)^{2}$ is included in $\mathbb{C}\left[\mathcal{M}_{\mathcal{C}}^{\text {abel }}\right]$ but absent from $\mathbb{C}\left[\mathcal{C}_{\text {abel }}\right]$. (A comparison between $\mathbb{C}\left[\mathcal{M}_{\mathcal{C}}^{\text {abel }}\right]$ and $\mathbb{C}\left[\mathcal{C}_{\text {abel }}\right]^{W_{G}}$ may appear to be fairer, since both objects are Weyl-symmetric, but it makes no difference since $\mathbb{C}\left[\mathcal{C}_{\text {abel }}\right]^{W_{G}} \leq \mathbb{C}\left[\mathcal{C}_{\text {abel }}\right]$ implies that the element in question does not belong to the Weyl-invariant ring $\mathbb{C}\left[\mathcal{C}_{\text {abel }}\right]^{W_{G}}$ either.)

We use $\mathbb{C}\left[\mathcal{C}_{\text {abel }}\right]$ as an active computational precursor to the Coulomb branch chiral ring and therefore reserve it the name abelianised ring despite any confusion it may cause when compared to the similarly denoted ring in [4].
} 
does contain an inverse mass as part of an expression. This is worrying because, as $\varphi_{2} \rightarrow \varphi_{1}$, this function diverges and so indicates that either the locus $\varphi_{1}=\varphi_{2}$ is not in the Coulomb branch (a solution which should be rejected on physical grounds), or else the function is not in the Coulomb branch chiral ring. The latter is correct: every element of $\mathbb{C}[\mathcal{C}]$ must be Weyl-invariant, and this one clearly is not. But that only raises another worry. Consider the Weyl-invariant polynomial

$$
u_{1}^{+} u_{1}^{-}+u_{2}^{+} u_{2}^{-}=-\frac{\prod_{i}\left(\varphi_{1}-M_{i}\right)}{\left(\varphi_{1}-\varphi_{2}\right)^{2}}-\frac{\prod_{i}\left(\varphi_{2}-M_{i}\right)}{\left(\varphi_{1}-\varphi_{2}\right)^{2}} .
$$

It also diverges as $\varphi_{2} \rightarrow \varphi_{1}$. Any workable prescription for reducing $\mathbb{C}\left[\mathcal{C}_{\text {abel }}\right]$ to $\mathbb{C}[\mathcal{C}]$ must exclude this polynomial from the ring. ${ }^{20}$

We are aware of two candidate prescriptions. Ref. [4] suggest in their section 6.3 that $\mathbb{C}[\mathcal{C}]$ can be generated as a Poisson algebra by a certain small set of Weyl-invariant operators, namely all single-node bare monopoles $\sum_{a} u_{i, a}^{ \pm}$and single-node elementary symmetric polynomials in $\varphi_{i, a}$, i.e. $\sum_{a} \varphi_{i, a}, \sum_{a<b} \varphi_{i, a} \varphi_{i, b}$ etc. We have observed its validity in every unitary quiver which we have studied in depth. In many cases an alternative method is available and we are able to define the Coulomb branch chiral ring by directly specifying its generators $^{21}$ in the abelianised formalism, with relations following directly from (2.30) as described in section 2.4. The two approaches have given identical results on every unitary quiver which we have studied.

First approach. Consider first the construction in [4] which only uses $u_{1}^{ \pm}+u_{2}^{ \pm}, \varphi_{1}+\varphi_{2}$ and $\varphi_{1} \varphi_{2}$ to generate the entire ring as a Poisson algebra. We wish to construct $u_{1}^{+} u_{1}^{-}+$ $u_{2}^{+} u_{2}^{-}$. There are only a few things one could try. Since the Poisson bracket $\{\cdot, \cdot\}$ has dimension -1 , the action of $\left\{\varphi_{1} \varphi_{2}, \cdot\right\}$ increases dimension by 1 while $\left\{\varphi_{1}+\varphi_{2}, \cdot\right\}$ and $\left\{u_{1}^{ \pm}+u_{2}^{ \pm}, \cdot\right\}$ preserve it. At the same time the space of $\Delta=2$ operators is finite so successive use of $\left\{\varphi_{1}+\varphi_{2}, \cdot\right\}$ will only bring finitely many new elements to consider. And we are only interested in operators of vanishing topological charge.

Let's start with $\left(u_{1}^{+}+u_{2}^{+}\right)\left(u_{1}^{-}+u_{2}^{-}\right)$which includes an extra contribution of $u_{1}^{+} u_{2}^{-}+$ $u_{2}^{+} u_{1}^{-}$; it must be removed to create (A.3). There is nothing else left to do without using the dimension-raising Poisson bracket:

$$
\begin{aligned}
\left\{\varphi_{1} \varphi_{2}, u_{1}^{+}+u_{2}^{+}\right\} & =u_{1}^{+} \varphi_{2}+u_{2}^{+} \varphi_{1} \\
\left\{u_{1}^{+} \varphi_{2}+u_{2}^{+} \varphi_{1}, u_{1}^{-}+u_{2}^{-}\right\} & =-\varphi_{1}^{2}-\varphi_{2}^{2}-\left(u_{1}^{+}+u_{2}^{+}\right)\left(u_{1}^{-}+u_{2}^{-}\right)
\end{aligned}
$$

This ring element does not help and it does not take much work to convince oneself that we have exhausted all non-trivial options, which implies the construction in [4] is free of the problematic element (A.3).

Second approach. We can also explicitly construct ring generators in representations of the moduli space symmetry. The Coulomb branch Hilbert series of this theory reads

$$
\operatorname{HS}(t)=1+t^{2}[2]+t^{4}([4]+[2]+[0])+\mathcal{O}\left(t^{3}\right)
$$

\footnotetext{
${ }^{20}$ The simple, but incorrect prescription $\mathbb{C}[\mathcal{C}]=\mathbb{C}\left[\mathcal{C}_{\text {abel }}\right]^{\mathcal{W}_{G}}$ would fail it. We thank an anonymous reviewer for bringing this to our attention.

${ }^{21}$ That is, generators of a ring, as opposed to a Poisson algebra.
} 
with plethystic logarithm

$$
P L(\mathrm{HS})(t)=t^{2}[2]+t^{4}[2]-t^{6}-t^{8}
$$

implying that its chiral ring is generated by six generators forming two adjoint representations [2] which we label $N$ and $R$. This representation forces constraints on any putative abelianised construction. For any $X, Y \in \mathfrak{s u}(2)$, expressed as square $2 \times 2$ matrices,

$$
\{\operatorname{tr}(N X), \operatorname{tr}(N Y)\}=\operatorname{tr}(N[X, Y])
$$

and

$$
\{\operatorname{tr}(N X), \operatorname{tr}(R Y)\}=\operatorname{tr}(R[X, Y]) .
$$

One can then use these constraints to find abelianised expressions of $N$ and $R$. In the massless case they are

$$
N=\left(\begin{array}{cc}
\varphi_{1}+\varphi_{2} & u_{1}^{-}+u_{2}^{-} \\
u_{1}^{+}+u_{2}^{+} & -\varphi_{1}-\varphi_{2}
\end{array}\right)
$$

(as in [7]) and

$$
R=\left(\begin{array}{cc}
-\frac{1}{2}\left(\varphi_{1}^{2}+\varphi_{2}^{2}+\left(u_{1}^{+}+u_{2}^{+}\right)\left(u_{1}^{-}+u_{2}^{-}\right)\right) & u_{1}^{-} \varphi_{2}+u_{2}^{-} \varphi_{1} \\
u_{1}^{+} \varphi_{2}+u_{2}^{+} \varphi_{1} & \frac{1}{2}\left(\varphi_{1}^{2}+\varphi_{2}^{2}+\left(u_{1}^{+}+u_{2}^{+}\right)\left(u_{1}^{-}+u_{2}^{-}\right)\right)
\end{array}\right) .
$$

One can check that the Lie algebra homomorphisms (A.6) and (A.7) are satisfied, and further that this choice of $N$, consistent with previous work, fixes the choice of $R$ up to a scalar factor (assuming the positively charged monopole is of the form $u^{+} \varphi$ ). One can also check that these two generators satisfy precisely the relations predicted by the Coulomb branch Hilbert series.

Agreement with the Hilbert series licences us to claim that components of $N$ and $R$ (two of which are redundant, for a total of 6 independent entries) generate $\mathbb{C}[\mathcal{C}]$. Observe that there is no way to generate eg. $\left(u_{1}^{+}\right)^{2}+\left(u_{2}^{+}\right)^{2}$ from the generators presented here. In fact the $\Delta=2$ subspace of $\mathbb{C}[\mathcal{C}]$ is generated by i) quadratic combinations of components of $N$ and ii) components of $R$. Notably, it is impossible to construct the counter-example (A.3) with these building blocks.

Comparison between the two approaches. It is interesting to compare the two approaches to reducing the abelianised ring to $\mathbb{C}[\mathcal{C}]$ : the new off-diagonal terms in $R$ appear as $\left\{\varphi_{1} \varphi_{2}, u_{1}^{ \pm}+u_{2}^{ \pm}\right\}$and diagonal terms are straightforwardly constructed from $\varphi_{1} \varphi_{2}$ and components of $N$ even without the use of a Poisson bracket.

In summary neither approach generates the divergent function (A.3). One can therefore interpret the appearance of inverse masses in (A.2) as a sign that we were dealing with the wrong variables and relations. The correct variables are i) elements of a certain Poisson algebra or ii) components of two coadjoint tensors, which themselves satisfy several tensorial relations. In the latter case, abelianised relations are crucial in deriving and testing the form of these coadjoint tensors, but ultimately serve as a ladder to be thrown away once the tensors are obtained. As mentioned above the two approaches agree on every unitary quiver we are aware of. 


\section{B Folded Lie algebras are the same as discretely gauged Lie algebras}

As mentioned in the main text, the Lie algebra of the discretely gauged space is given by

$$
\left\{\tilde{\mathcal{O}}_{i}, \tilde{\mathcal{O}}_{j}\right\}=\sum_{k} c_{i j}{ }^{k} \tilde{\mathcal{O}}_{k}
$$

for $\tilde{\mathcal{O}}_{i}$ which form a basis of $\mathbb{C}[\tilde{\mathcal{C}}]_{2}$. In particular these operators vary across the moduli space. Restricting to the folded subspace, we find

$$
\left\{\hat{\mathcal{O}}_{i}, \hat{\mathcal{O}}_{j}\right\}=\sum_{k} c_{i j}{ }^{k} \hat{\mathcal{O}}_{k}
$$

This does not necessarily mean that the two Lie algebras are isomorphic as some of the r.h.s. terms could vanish if $\hat{\mathcal{O}}_{k}$ vanishes identically. We will now prove that this does not happen.

$\tilde{\mathcal{O}}_{k}$ is a non-constant symmetric function in variables attached to wreathed legs; call them $\vec{x}_{i}$ where $i$ labels the leg. So we can rewrite the operator as $f\left(\vec{x}_{1}, \ldots \vec{x}_{n}\right)$ for some $n$. At the fixed point $\vec{x}_{i}=\vec{x}$, so the operator becomes $f(\vec{x}, \ldots, \vec{x})$. Assume it vanishes everywhere. Then

$$
\nabla_{\vec{x}} f(\vec{x}, \ldots, \vec{x})=\left.\sum_{i} \nabla_{\vec{x}_{i}} f\left(\vec{x}_{1}, \ldots \vec{x}_{n}\right)\right|_{\vec{x}_{j}=x} .
$$

However all the summands are identical under the restriction:

$$
\begin{aligned}
\left(\nabla_{\vec{x}_{i}} f\left(\vec{x}_{1}, \ldots \vec{x}_{n}\right)\right)_{j} & =\lim _{\varepsilon \rightarrow 0} \frac{f\left(\vec{x}_{1}, \ldots, \vec{x}_{i}+\varepsilon e_{i}, \ldots, \vec{x}_{n}\right)-f\left(\vec{x}_{1}, \ldots, \vec{x}_{i}, \ldots, \vec{x}_{n}\right)}{\varepsilon} \\
& =\lim _{\varepsilon \rightarrow 0} \frac{f\left(\vec{x}_{i}+\varepsilon e_{i}, \ldots, \vec{x}_{1}, \ldots, \vec{x}_{n}\right)-f\left(\vec{x}_{i}, \ldots, \vec{x}_{1}, \ldots \vec{x}_{n}\right)}{\varepsilon} \\
& =\left(\nabla_{\vec{x}_{1}^{\prime}} f\left(\vec{x}_{1}^{\prime}, \ldots \vec{x}_{n}^{\prime}\right)\right)_{j} \mid \begin{array}{c}
\vec{x}_{1}^{\prime}=\vec{x}_{i} \\
\vec{x}_{i}^{\prime}=\vec{x}_{1} \\
\vec{x}_{j \neq 1, i}^{\prime}=\vec{x}_{j}
\end{array}
\end{aligned}
$$

so

$$
\left.\left(\nabla_{\vec{x}_{i}} f\left(\vec{x}_{1}, \ldots \vec{x}_{n}\right)\right)_{j}\right|_{\vec{x}_{j}=\vec{x}}=\left.\left(\nabla_{\vec{x}_{1}} f\left(\vec{x}_{1}, \ldots \vec{x}_{n}\right)\right)_{j}\right|_{\vec{x}_{j}=\vec{x}}
$$

Then

$$
\nabla_{\vec{x}} f(\vec{x}, \ldots, \vec{x})=\left.n \nabla_{\vec{x}_{1}} f\left(\vec{x}_{1}, \ldots \vec{x}_{n}\right)\right|_{\vec{x}_{j}=x} \neq 0
$$

unless $\left.\nabla_{\vec{x}_{i}} f\left(\vec{x}_{1}, \ldots \vec{x}_{n}\right)\right|_{\vec{x}_{j}=\vec{x}}$ vanishes, ie. $f\left(\vec{x}_{1}, \ldots \vec{x}_{n}\right)$ is a constant, which contradicts the assumption that $\tilde{\mathcal{O}}_{k}$ is non-constant. It follows that both the discretely gauged and folded spaces have isomorphic Lie algebras and hence share the same continuous symmetry. 


\section{Computation of Hilbert series with $S_{4}$ wreathing}

The computation of the exact Hilbert series presented in figure 4 can be done in principle using (3.44). However it is often useful to massage this formula until a more manageable form can be used in practice. In this appendix, we give the result of such manipulations in the case of the quiver at hand. Derivations use simple algebra and are not detailed here.

Using the notations of (3.48), but using the gauge group (3.55), one can set $m_{a, 2}=0$ and $m_{a, 1} \equiv m_{a}$ and the conformal dimension can be expressed in terms of

$$
m=\left(m_{a}, m_{b}, m_{c}, m_{d}, m_{e}\right)
$$

as

$2 \Delta(m)=\left|m_{a}-m_{b}\right|+\left|m_{a}-m_{c}\right|+\left|m_{a}-m_{d}\right|+\left|m_{a}-m_{e}\right|+\left|m_{b}\right|+\left|m_{c}\right|+\left|m_{d}\right|+\left|m_{e}\right|-2\left|m_{a}\right|$.

One then computes the auxiliary sums

$$
\Sigma_{i}=\sum_{m_{a}=0}^{\infty} \sum_{\left(m_{b}, m_{c}, m_{d}, m_{e}\right) \in \text { Range }_{i}} t^{2 \Delta(m)}
$$

where Range ${ }_{i}$ is defined in table 15 . The exact value of the sums $\Sigma_{i}$ is straightforward to compute (note the absence of Casimir factors!) and is given in table 15 as well.

Let's now pick a subgroup $\Gamma$ of $S_{4}$. For $\mu \in \mathbb{Z}^{4}$ we call $O^{S_{4}}(\mu)$ the orbit of $\mu$ under the action of $S_{4}$. This orbit can be written as a disjoint union of $n(\mu)$ orbits under $\Gamma$,

$$
O^{S_{4}}(\mu)=\coprod_{j=1}^{n(\mu)} O^{\Gamma}\left(\mu_{j}\right)
$$

where the $\mu_{j} \in \mathbb{Z}^{4}$ are representatives of these orbits, (not uniquely!) determined by the above equation. Using the notation (3.46), that we recall here,

$$
P_{\Gamma}\left(t^{2} ; \mu\right)=\frac{1}{|\Gamma|} \sum_{\gamma \in \Gamma(\mu)} \frac{1}{\operatorname{det}\left(1-t^{2} \gamma\right)},
$$

one can define the modified Casimir factor

$$
\tilde{P}_{\Gamma}\left(t^{2} ; \mu\right)=\sum_{j=1}^{n(\mu)} P_{\Gamma}\left(t^{2} ; \mu_{j}\right) .
$$

The rationale behind this definition is that we have evaluated the sums (C.3) which are adapted to the full group $S_{4}$, and the Casimir factors for the group $\Gamma$ have to be collected accordingly. This being done, the Hilbert series for the Coulomb branch of the wreathed quivers are simply

$$
\operatorname{HS}_{\Gamma}(t)=\sum_{i=1}^{6} \tilde{P}_{\Gamma}\left(t^{2} ; \mu_{i}\right) \Sigma_{i},
$$

where $\mu_{i} \in \mathbb{Z}^{4}$ is any element satisfying the condition Range ${ }_{i}$. Using this formula, all the Hilbert series of figure 4 are evaluated in a fraction of a second on a standard computer. 


\begin{tabular}{|c|c|c|}
\hline$i$ & Range $_{i}$ & $\Sigma_{i}$ \\
\hline 1 & $m_{b}<m_{c}<m_{d}<m_{e}$ & $\frac{t^{6}\left(4+16 t^{2}+18 t^{4}+13 t^{6}+4 t^{8}+t^{10}\right)}{\left(1-t^{2}\right)^{6}\left(1+t^{2}\right)^{3}}$ \\
\hline 2 & $\begin{array}{l}m_{b}=m_{c}<m_{d}<m_{e} \\
m_{b}<m_{c}<m_{d}=m_{e}\end{array}$ & $\frac{t^{4}\left(2+9 t^{2}+10 t^{4}+9 t^{6}+3 t^{8}+t^{10}\right)}{\left(1-t^{2}\right)^{5}\left(1+t^{2}\right)^{3}}$ \\
\hline 3 & $m_{b}<m_{c}=m_{d}<m_{e}$ & $\frac{t^{4}\left(4+7 t^{2}+7 t^{4}+3 t^{6}+t^{8}\right)}{\left(1-t^{2}\right)^{5}\left(1+t^{2}\right)^{2}}$ \\
\hline 4 & $\begin{array}{l}m_{b}=m_{c}=m_{d}<m_{e} \\
m_{b}<m_{c}=m_{d}=m_{e}\end{array}$ & $\frac{t^{2}\left(2+3 t^{2}+4 t^{4}+2 t^{6}+t^{8}\right)}{\left(1-t^{2}\right)^{4}\left(1+t^{2}\right)^{2}}$ \\
\hline 5 & $m_{b}=m_{c}<m_{d}=m_{e}$ & $\frac{t^{2}+5 t^{4}+5 t^{6}+6 t^{8}+2 t^{10}+t^{12}}{\left(1-t^{2}\right)^{4}\left(1+t^{2}\right)^{3}}$ \\
\hline 6 & $m_{b}=m_{c}=m_{d}=m_{e}$ & $\frac{\left(1-t+t^{2}\right)\left(1+t+t^{2}\right)\left(1+t^{4}\right)}{\left(1-t^{2}\right)^{3}\left(1+t^{2}\right)^{2}}$ \\
\hline
\end{tabular}

Table 15. Definitions of the ranges involved in the sums (C.3), and exact values of these sums. When there are two possible ranges, this means that the two choices lead to the same sums.

Open Access. This article is distributed under the terms of the Creative Commons Attribution License (CC-BY 4.0), which permits any use, distribution and reproduction in any medium, provided the original author(s) and source are credited.

\section{References}

[1] S. Cremonesi, G. Ferlito, A. Hanany and N. Mekareeya, Coulomb branch and the moduli space of instantons, JHEP 12 (2014) 103 [arXiv:1408.6835] [INSPIRE].

[2] S. Cremonesi, A. Hanany and A. Zaffaroni, Monopole operators and Hilbert series of Coulomb branches of $3 d N=4$ gauge theories, JHEP 01 (2014) 005 [arXiv:1309.2657] [INSPIRE].

[3] H. Nakajima and A. Weekes, Coulomb branches of quiver gauge theories with symmetrizers, arXiv: 1907.06552 [INSPIRE].

[4] M. Bullimore, T. Dimofte and D. Gaiotto, The Coulomb branch of $3 d N=4$ theories, Commun. Math. Phys. 354 (2017) 671 [arXiv:1503.04817] [INSPIRE].

[5] A. Hanany and A. Zajac, Discrete gauging in Coulomb branches of three dimensional $N=4$ supersymmetric gauge theories, JHEP 08 (2018) 158 [arXiv: 1807.03221] [INSPIRE].

[6] A. Hanany and M. Sperling, Discrete quotients of 3-dimensional $N=4$ Coulomb branches via the cycle index, JHEP 08 (2018) 157 [arXiv: 1807.02784] [INSPIRE].

[7] A. Hanany and D. Miketa, Nilpotent orbit Coulomb branches of types AD, JHEP 02 (2019) 113 [arXiv: 1807.11491] [INSPIRE].

[8] N. Marcus and A. Sagnotti, Tree level constraints on gauge groups for type I superstrings, Phys. Lett. B 119 (1982) 97 [InSPIRE].

[9] L.J. Dixon, J.A. Harvey, C. Vafa and E. Witten, Strings on orbifolds, Nucl. Phys. B 261 (1985) 678 [INSPIRE].

[10] L.J. Dixon, J.A. Harvey, C. Vafa and E. Witten, Strings on orbifolds. 2, Nucl. Phys. B 274 (1986) 285 [INSPIRE].

[11] A. Dabholkar, Lectures on orientifolds and duality, in ICTP summer school in high-energy physics and cosmology, (1997), pg. 128 [hep-th/9804208] [INSPIRE]. 
[12] A. Sen, Duality and orbifolds, Nucl. Phys. B 474 (1996) 361 [hep-th/9604070] [InSPIRE].

[13] A. Bourget et al., The Higgs mechanism - Hasse diagrams for symplectic singularities, JHEP 01 (2020) 157 [arXiv: 1908. 04245] [INSPIRE].

[14] P.N. Achar and A. Henderson, Geometric Satake, Springer correspondence and small representations, Selecta Math. 19 (2013) 949 [arXiv:1108.4999].

[15] A. Bourget, J.F. Grimminger, A. Hanany, M. Sperling and Z. Zhong, The affine Grassmannian, quivers and branes, to appear (2021).

[16] A. Braverman, M. Finkelberg and H. Nakajima, Coulomb branches of $3 d N=4$ quiver gauge theories and slices in the affine Grassmannian, Adv. Theor. Math. Phys. 23 (2019) 75 [arXiv: 1604.03625] [INSPIRE].

[17] H. Nakajima, Towards a mathematical definition of Coulomb branches of 3-dimensional $N=4$ gauge theories, I, Adv. Theor. Math. Phys. 20 (2016) 595 [arXiv:1503.03676] [INSPIRE].

[18] A. Braverman, M. Finkelberg and H. Nakajima, Towards a mathematical definition of Coulomb branches of 3-dimensional $N=4$ gauge theories, II, Adv. Theor. Math. Phys. 22 (2018) 1071 [arXiv:1601.03586] [INSPIRE].

[19] A. Hanany and A. Zajac, Ungauging schemes and Coulomb branches of non-simply laced quiver theories, JHEP 09 (2020) 193 [arXiv:2002.05716] [INSPIRE].

[20] R. Brylinski and B. Kostant, Nilpotent orbits, normality, and hamiltonian group actions, J. Amer. Math. Soc. 7 (1994) 269.

[21] P.Z. Kobak and A. Swann, Classical nilpotent orbits as hyper-Kähler quotients, Int. J. Math. 07 (1996) 193.

[22] D. Bump, Lie groups, Springer, New York, NY, U.S.A. (2004).

[23] A. Hanany and R. Kalveks, Quiver theories and formulae for nilpotent orbits of exceptional algebras, JHEP 11 (2017) 126 [arXiv:1709.05818] [INSPIRE].

[24] Y. Namikawa, A characterization of nilpotent orbit closures among symplectic singularities, Math. Annalen 370 (2017) 811.

[25] A. Joseph, The minimal orbit in a simple Lie algebra and its associated maximal ideal, Ann. Sci. École Norm. Sup. 9 (1976) 1.

[26] D. Gaiotto, A. Neitzke and Y. Tachikawa, Argyres-Seiberg duality and the Higgs branch, Commun. Math. Phys. 294 (2010) 389 [arXiv:0810.4541] [INSPIRE].

[27] M. Dedushenko, Y. Fan, S.S. Pufu and R. Yacoby, Coulomb branch operators and mirror symmetry in three dimensions, JHEP 04 (2018) 037 [arXiv:1712.09384] [INSPIRE].

[28] M. Dedushenko, Y. Fan, S.S. Pufu and R. Yacoby, Coulomb branch quantization and Abelianized monopole bubbling, JHEP 10 (2019) 179 [arXiv:1812.08788] [INSPIRE].

[29] A. Kerber, Applied finite group actions, Springer, Berlin, Heidelberg, Germany (1999).

[30] A. Hanany and R. Kalveks, Quiver theories for moduli spaces of classical group nilpotent orbits, JHEP 06 (2016) 130 [arXiv:1601.04020] [INSPIRE].

[31] G. Cheng, A. Hanany, Y. Li and Y. Zhao, Coulomb branch for A-type balanced quivers in $3 d$ $N=4$ gauge theories, arXiv:1701.03825 [INSPIRE].

[32] S. Lee, Discrete gauging: weight maps, finite groups and multiple nodes, unpublished Summer report (2018). 
[33] A. Bourget and A. Pini, Non-connected gauge groups and the plethystic program, JHEP 10 (2017) 033 [arXiv : 1706.03781] [INSPIRE].

[34] R. Wendt, Weyl's character formula for non-connected Lie groups and orbital theory for twisted affine Lie algebras, J. Funct. Anal. 180 (2001) 31.

[35] A. Hanany and R. Kalveks, Quiver theories and Hilbert series of classical slodowy intersections, Nucl. Phys. B 952 (2020) 114939 [arXiv:1909.12793] [INSPIRE].

[36] R. Yamagishi, Four-dimensional conical symplectic hypersurfaces, J. Alg. 560 (2020) 538.

[37] A. Hanany and N. Mekareeya, Tri-vertices and SU(2)'s, JHEP 02 (2011) 069 [arXiv: 1012.2119] [INSPIRE].

[38] M. Finkelberg and E. Goncharov, Coulomb branch of a multiloop quiver gauge theory, Funct. Anal. Appl. 53 (2019) 241 [arXiv: 1903. 05822] [INSPIRE].

[39] K.A. Intriligator and N. Seiberg, Mirror symmetry in three-dimensional gauge theories, Phys. Lett. B 387 (1996) 513 [hep-th/9607207] [INSPIRE].

[40] B. Feng and A. Hanany, Mirror symmetry by O3-planes, JHEP 11 (2000) 033 [hep-th/0004092] [INSPIRE].

[41] D. Gaiotto and E. Witten, S-duality of boundary conditions in $N=4$ super Yang-Mills theory, Adv. Theor. Math. Phys. 13 (2009) 721 [arXiv:0807.3720] [INSPIRE].

[42] A. Hanany, N. Mekareeya and S.S. Razamat, Hilbert series for moduli spaces of two instantons, JHEP 01 (2013) 070 [arXiv:1205.4741] [INSPIRE].

[43] A. Dey, A. Hanany, P. Koroteev and N. Mekareeya, On three-dimensional quiver gauge theories of type B, JHEP 09 (2017) 067 [arXiv: 1612.00810] [INSPIRE].

[44] N. Haouzi and C. Kozçaz, The ABCDEFG of little strings, arXiv:1711.11065 [INSPIRE].

[45] N. Haouzi and C. Kozçaz, Supersymmetric Wilson loops, instantons, and deformed $W$-algebras, arXiv:1907.03838 [INSPIRE].

[46] T. Kimura and V. Pestun, Fractional quiver W-algebras, Lett. Math. Phys. 108 (2018) 2425 [arXiv: 1705.04410] [INSPIRE].

[47] G. Zafrir, Compactifications of 5d SCFTs with a twist, JHEP 01 (2017) 097 [arXiv: 1605.08337] [INSPIRE].

[48] K. Ohmori, Y. Tachikawa and G. Zafrir, Compactifications of $6 d N=(1,0)$ SCFTs with non-trivial Stiefel-Whitney classes, JHEP 04 (2019) 006 [arXiv:1812.04637] [INSPIRE].

[49] S. Cabrera, A. Hanany and M. Sperling, Magnetic quivers, Higgs branches, and $6 d N=(1,0)$ theories, JHEP 06 (2019) 071 [Erratum ibid. 07 (2019) 137] [arXiv: 1904.12293] [INSPIRE].

[50] A. Hanany and A. Pini, HWG for Coulomb branch of $3 d$ Sicilian theory mirrors, arXiv: 1707.09784 [INSPIRE].

[51] B. Fu, D. Juteau, P. Levy and E. Sommers, Generic singularities of nilpotent orbit closures, Adv. Math. 305 (2017) 1.

[52] A. Malkin, V. Ostrik and M. Vybornov, The minimal degeneration singularities in the affine Grassmannians, Duke Math. J. 126 (2005) 233 [math. AG/0305095].

[53] S. Cabrera, A. Hanany and Z. Zhong, Nilpotent orbits and the Coulomb branch of $T^{\sigma}(G)$ theories: special orthogonal vs orthogonal gauge group factors, JHEP 11 (2017) 079 [arXiv: 1707.06941] [INSPIRE]. 